EWA ZADRZYŃsKa (Warszawa)

WOJCIECH M. ZAJĄCZKOWsKI (Warszawa)

\title{
ON AN INEQUALITY FOR A FREE BOUNDARY PROBLEM FOR EQUATIONS OF A VISCOUS COMPRESSIBLE HEAT-CONDUCTING CAPILLARY FLUID
}

Abstract. We derive an inequality for a local solution of a free boundary problem for a viscous compressible heat-conducting capillary fluid. This inequality is crucial to proving the global existence of solutions belonging to certain anisotropic Sobolev-Slobodetskiı spaces and close to an equilibrium state.

1. Introduction. In the paper we obtain some crucial inequality for a local solution of the equations of motion of a viscous compressible heatconducting capillary fluid bounded by a free surface. The motion of such a fluid in a bounded domain $\Omega_{t} \subset \mathbb{R}^{3}$ (which depends on time $t \in \mathbb{R}_{+}$) is described by the following system with the boundary and initial conditions (see [6], [7]):

$$
\begin{array}{ll}
\varrho\left[v_{t}+(v \cdot \nabla) v\right]-\operatorname{div} \mathbb{T}(v, p)=0 & \text { in } \widetilde{\Omega}^{T}, \\
\varrho_{t}+\operatorname{div}(\varrho v)=0 & \text { in } \widetilde{\Omega}^{T}, \\
\varrho c_{v}\left(\theta_{t}+v \cdot \nabla \theta\right)+\theta p_{\theta} \operatorname{div} v-\varkappa \Delta \theta & \\
\quad-\frac{\mu}{2} \sum_{i, j=1}^{3}\left(v_{i x_{j}}+v_{j x_{i}}\right)^{2}-(\nu-\mu)(\operatorname{div} v)^{2}=\varrho r & \text { in } \widetilde{\Omega}^{T}, \\
\mathbb{T} \bar{n}-\sigma H \bar{n}=-p_{0} \bar{n} & \text { on } \widetilde{S}^{T}, \\
v \cdot \bar{n}=-\varphi_{t} /|\nabla \varphi| & \text { on } \widetilde{S}^{T},
\end{array}
$$

2000 Mathematics Subject Classification: 35Q35, 35R35, 76N10.

Key words and phrases: free boundary, compressible viscous heat-conducting fluid, surface tension.

Research supported by KBN grant no 2PO3A-003-14. 


$$
\begin{array}{ll}
\partial \theta / \partial n=\bar{\theta} & \text { on } \widetilde{S}^{T}, \\
\left.\varrho\right|_{t=0}=\varrho_{0},\left.\quad \theta\right|_{t=0}=\theta_{0},\left.\quad v\right|_{t=0}=v_{0} & \text { in } \Omega,
\end{array}
$$

where $\widetilde{\Omega}^{T} \equiv \bigcup_{t \in(0, T)} \Omega_{t} \times\{t\}, \Omega_{0}=\Omega$ is the initial domain, $\varphi(x, t)=0$ describes $S_{t}$ (at least locally), $\widetilde{S}^{T} \equiv \bigcup_{t \in(0, T)} S_{t} \times\{t\}, \bar{n}$ is the unit outward vector normal to the boundary, i.e. $\bar{n}=\nabla \varphi /|\nabla \varphi|$. Moreover, $v=v(x, t)$ is the velocity of the fluid, $\varrho=\varrho(x, t)$ the density, $\theta=\theta(x, t)$ the temperature, $r=r(x, t)$ the heat sources per unit mass, $\bar{\theta}=\bar{\theta}(x, t)$ the heat flow per unit surface, $p=p(\varrho, \theta)$ the pressure, $c_{v}=c_{v}(\varrho, \theta)$ the specific heat at constant volume, $\mu$ and $\nu$ the viscosity coefficients, $\varkappa$ the coefficient of heatconductivity, $\sigma$ the constant coefficient of surface tension, and $p_{0}$ the external (constant) pressure.

From the thermodynamic considerations we have

$$
\nu>\frac{1}{3} \mu>0, \quad \varkappa>0, \quad c_{v}>0, \quad \sigma>0 .
$$

Further, $\mathbb{T}=\mathbb{T}(v, p)$ denotes the stress tensor of the form

$$
\mathbb{T}(v, p)=\left\{T_{i j}\right\}_{i, j=1,2,3}=\left\{\mu S_{i j}(v)+(\nu-\mu) \delta_{i j} \operatorname{div} v-p \delta_{i j}\right\}_{i, j=1,2,3},
$$

where $\mathbb{S}(v)=\left\{v_{i x_{j}}+v_{j x_{i}}\right\}_{i, j=1,2,3}$ is the velocity deformation tensor.

Finally, we denote by $H$ the double mean curvature of $S_{t}$ which is negative for convex domains and can be expressed in the form

$$
H \bar{n}=\Delta_{S_{t}}(t) x, \quad x=\left(x_{1}, x_{2}, x_{3}\right),
$$

where $\Delta_{S_{t}}(t)$ is the Laplace-Beltrami operator on $S_{t}$. Let $S_{t}$ be determined by $x=x\left(s_{1}, s_{2}, t\right),\left(s_{1}, s_{2}\right) \in U \subset \mathbb{R}^{2}$. Then we have

$$
\Delta_{S_{t}}(t)=g^{-1 / 2} \frac{\partial}{\partial s_{\gamma}}\left(g^{1 / 2} g^{\gamma \delta} \frac{\partial}{\partial s_{\delta}}\right) \quad(\gamma, \delta=1,2),
$$

where the convention summation over repeated indices is assumed, $g=$ $\operatorname{det}\left\{g_{\gamma \delta}\right\}_{\gamma, \delta=1,2}, g_{\gamma \delta}=\frac{\partial x}{\partial s_{\gamma}} \cdot \frac{\partial x}{\partial s_{\delta}}$, and $\left\{g^{\gamma \delta}\right\}$ is the inverse matrix to $\left\{g_{\gamma \delta}\right\}$.

Assume that the domain $\Omega$ is given. Then by $(1.1)_{5}, \Omega_{t}=\left\{x \in \mathbb{R}^{3}: x=\right.$ $x(\xi, t), \xi \in \Omega\}$, where $x=x(\xi, t)$ is the solution of the Cauchy problem

$$
\frac{d x}{d t}=v(x, t),\left.\quad x\right|_{t=0}=\xi \in \Omega, \quad \xi=\left(\xi_{1}, \xi_{2}, \xi_{3}\right) .
$$

Integrating (1.2) we obtain

$$
x=\xi+\int_{0}^{t} u\left(\xi, t^{\prime}\right) d t^{\prime} \equiv X_{u}(\xi, t),
$$

where $u(\xi, t)=v\left(X_{u}(\xi, t)\right)$ and $x=X_{u}(\xi, t)$ describes the relation between the Eulerian $x$ and Lagrangian $\xi$ coordinates. Moreover, by $(1.1)_{5}, S_{t}=\{x$ : $x=x(\xi, t), \xi \in S=\partial \Omega\}$. 
By the continuity equation $(1.1)_{2}$ and the kinematic condition $(1.1)_{5}$ the total mass is conserved, i.e.

$$
\int_{\Omega_{t}} \varrho(x, t) d x=\int_{\Omega} \varrho_{0}(\xi) d \xi=M .
$$

Now, assume that $p_{\varrho}>0, p_{\theta}>0$ for $\varrho, \theta \in \mathbb{R}_{+}$and consider the equation

$$
p\left(\frac{M}{\frac{4}{3} \pi R_{e}^{3}}, \theta_{e}\right)=p_{0}+\frac{2 \sigma}{R_{e}} .
$$

We assume that there exist $R_{e}>0$ and $\theta_{e}>0$ satisfying (1.3). Then we have the following definition.

Definition 1.1. Let $r=\bar{\theta}=0$. By an equilibrium state we mean a solution $\left(v, \theta, \varrho, \Omega_{t}\right)$ of problem (1.1) such that $v=0, \theta=\theta_{e}, \varrho=\varrho_{e}$, $\Omega_{t}=\Omega_{e}$ for $t \geq 0$, where $\varrho_{\mathrm{e}}=M /\left(\frac{4}{3} \pi R_{e}^{3}\right), \Omega_{e}$ is a ball of radius $R_{e}$, and $R_{e}>0$ and $\theta_{e}>0$ satisfy equation (1.3).

Next, we introduce

$$
\varrho_{\sigma}=\varrho-\varrho_{e}, \quad \theta_{\sigma}=\theta-\theta_{e}, \quad p_{\sigma}=p-\frac{2 \sigma}{R_{e}}-p_{0} .
$$

We can write $p_{\sigma}$ in the form

$$
p_{\sigma}(\varrho, \theta)=p_{1} \varrho_{\sigma}+p_{2} \theta_{\sigma},
$$

where

$$
p_{1}(\varrho, \theta)=\int_{0}^{1} p_{\varrho}\left(\varrho_{e}+s\left(\varrho-\varrho_{e}\right), \theta\right) d s, \quad p_{2}(\theta)=\int_{0}^{1} p_{\theta}\left(\varrho_{e}, \theta_{e}+s\left(\theta-\theta_{e}\right)\right) d s .
$$

Then by using (1.4) problem (1.1) can be rewritten in Lagrangian coordinates as follows:

$$
\begin{array}{ll}
\eta u_{t}-\operatorname{div}_{u} \mathbb{T}_{u}\left(u, p_{\sigma}\right)=0 & \text { in } \Omega^{T} \equiv \Omega \times(0, T), \\
\eta_{\sigma t}+\eta \operatorname{div}_{u} u=0 & \text { in } \Omega^{T}, \\
\eta c_{v} \vartheta_{\sigma t}+\vartheta p_{\vartheta} \operatorname{div}_{u} u-\varkappa \nabla_{u}^{2} \vartheta_{\sigma} & \\
\quad=\frac{\mu}{2} \sum_{i, j=1}^{3}\left(\xi_{x_{i}} \cdot \nabla_{\xi} u_{j}+\xi_{x_{j}} \cdot \nabla_{\xi} u_{i}\right)^{2} & \\
\quad-(\nu-\mu)\left(\operatorname{div}_{u} u\right)^{2}+\eta k & \text { in } \Omega^{T}, \\
\mathbb{T}_{u}\left(u, p_{\sigma}\right) \bar{n}_{u}-\sigma\left(H+2 / R_{e}\right) \bar{n}_{u}=0 & \text { on } S^{T} \equiv S \times(0, T), \\
\bar{n}_{u} \cdot \nabla_{u} \vartheta_{\sigma}=\bar{\vartheta} & \text { on } S^{T}, \\
\left.\eta_{\sigma}\right|_{t=0}=\varrho_{\sigma 0},\left.\quad \vartheta_{\sigma}\right|_{t=0}=\theta_{\sigma 0},\left.\quad u\right|_{t=0}=v_{0} & \text { in } \Omega,
\end{array}
$$

where $\eta(\xi, t)=\varrho\left(X_{u}(\xi, t), t\right), \vartheta(\xi, t)=\theta\left(X_{u}(\xi, t), t\right), \eta_{\sigma}=\eta-\varrho_{e}, \vartheta_{\sigma}=\vartheta-$ $\theta_{e}, k(\xi, t)=r\left(X_{u}(\xi, t), t\right), \bar{\vartheta}(\xi, t)=\bar{\theta}\left(X_{u}(\xi, t), t\right), \bar{n}_{u}(\xi, t)=\bar{n}\left(X_{u}(\xi, t), t\right)$, 


$$
\begin{aligned}
\nabla_{u}=\xi_{i x} \partial_{\xi_{i}} & =\left\{\xi_{i x_{j}} \partial_{\xi_{i}}\right\}_{j=1,2,3}, \mathbb{T}_{u}(u, p)=-p I+\mathbb{D}_{u}(u), I=\left\{\delta_{i j}\right\}_{i, j=1,2,3}, \\
\mathbb{D}_{u}(u)= & \left\{\mathbb{D}_{u i j}(u)\right\}_{i, j=1,2,3} \\
= & \left\{\mu\left(\partial_{x_{i}} \xi_{k} \partial_{\xi_{k}} u_{j}+\partial_{x_{j}} \xi_{k} \partial_{\xi_{k}} u_{i}\right)+(\nu-\mu) \delta_{i j} \operatorname{div}_{u} u\right\}_{i, j=1,2,3},
\end{aligned}
$$

$\operatorname{div}_{u} u=\nabla_{u} \cdot u=\partial_{x_{i}} \xi_{k} \partial_{\xi_{k}} u_{i}, \operatorname{div}_{u} \mathbb{T}_{u}(u, p)=\left\{\partial_{x_{j}} \xi_{k} \partial_{\xi_{k}} T_{u i j}(u, p)\right\}_{i=1,2,3}$ and $\partial_{x_{i}} \xi_{k}$ are the elements of the matrix $\xi_{x}$ which is inverse to the matrix $x_{\xi}=I+\int_{0}^{t} u_{\xi}\left(\xi, t^{\prime}\right) d t^{\prime}$.

The aim of our considerations is to prove the global existence of solutions to problem (1.1) which are sufficiently close to the equilibrium solution (see Definition 1.1) for all $t>0$. The result is stated as follows:

Theorem (see [12]). Let

$$
\begin{aligned}
& \left\|v_{0}\right\|_{1+\alpha, \Omega}+\left\|\varrho_{\sigma 0}\right\|_{1+\alpha, \Omega}+\left\|\theta_{\sigma 0}\right\|_{1+\alpha, \Omega} \leq \delta_{1}, \\
& \|r\|_{C_{B}^{2}\left(\mathbb{R}^{3} \times(0, \infty)\right)}+\|\bar{\theta}\|_{C_{B}^{3}\left(\mathbb{R}^{3} \times(0, \infty)\right)} \leq \delta_{2}, \\
& \int_{\Omega} \varrho_{0} d \xi=M, \quad \int_{\Omega} \varrho_{0} \xi d \xi=0, \quad \int_{\Omega} \varrho_{0} v_{0} d \xi=0 .
\end{aligned}
$$

Assume that the initial domain $\Omega$ is close to a ball and $S$ is described by $|x|=R(\omega)$, where $\omega \in S^{1}$ ( $S^{1}$ is the unit sphere). Let

$$
\left\|R(\omega)-R_{e}\right\|_{1, S^{1}} \leq \delta_{3}, \quad\left\|H(\cdot, 0)+2 / R_{e}\right\|_{1+\alpha / 2, S} \leq \delta_{4} .
$$

Let $\alpha \in(3 / 4,1)$ and assume that the data satisfy some other conditions which are implied by conservation laws and which are described in [12]. Then for sufficiently small $\delta_{i}(i=1, \ldots, 4)$ the local solution to problem (1.1) (see [13]) can be prolonged infinitely in time.

The main role in the process of prolonging the local solution in time is played by inequality (3.1) of Section 3. The paper is devoted to showing it.

To prove the theorem we also need a global energy estimate and an estimate showing the increase of regularity of the solution after some time. Both have been derived in [14]. However, the latter estimate has been obtained in the case of $r=\bar{\theta}=0$. For nonvanishing $r$ and $\bar{\theta}$ such an estimate is shown in Section 4 of this paper (see Theorem 4.1).

The global existence result is proved in spaces such that $\left(u, \vartheta_{\sigma}, \eta_{\sigma}\right) \in$ $W_{2}^{2+\alpha, 1+\alpha / 2}\left(\Omega_{k T} \times(k T,(k+1) T)\right) \times W_{2}^{2+\alpha, 1+\alpha / 2}\left(\Omega_{k T} \times(k T,(k+1) T)\right) \times$ $C\left([k T,(k+1) T] ; W_{2}^{1+\alpha}\left(\Omega_{k T}\right)\right) \cap W_{2}^{1+\alpha, 1+\alpha / 2}\left(\Omega_{k T} \times(k T,(k+1) T)\right)$, where $k \in \mathbb{N} \cup\{0\} ; u, \eta_{\sigma}, \vartheta_{\sigma}$ denote $v, \varrho_{\sigma}, \theta_{\sigma}$ written in Lagrangian coordinates $\xi \in \Omega_{k T}$; and $T$ is the time of local existence.

We use the spaces with fractional derivatives to prove existence of solutions with the lowest possible regularity. In the compressible case (see [9], [10], [5]) it was possible to prove the existence in classes of functions such that $\alpha \in(1 / 2,1)$. However, in the general heat-conducting case we need 
to assume that $\alpha \in(3 / 4,1)$. This restriction is connected with the strong nonlinearity of equation $(1.1)_{3}$.

We derive (3.1) for the local solution $\left(u, \vartheta_{\sigma}, \eta_{\sigma}\right) \in W_{2}^{2+\alpha, 1+\alpha / 2}\left(\Omega^{T}\right) \times$ $W_{2}^{2+\alpha, 1+\alpha / 2}\left(\Omega^{T}\right) \times W_{2}^{1+\alpha, 1 / 2+\alpha / 2}\left(\Omega^{T}\right) \cap C\left([0, T] ; W_{2}^{1+\alpha}(\Omega)\right), \alpha \in(3 / 4,1)$ (see definitions in Section 2) in which the norms of $u, \vartheta_{\sigma}, \eta_{\sigma}$ are estimated by the sum of the $L_{2}$-norms of these functions, the nonlinear terms consisting of the products of the highest order norms of $u, \vartheta_{\sigma}, \eta_{\sigma}$ and the norms of data. The most important feature of the inequality is that on the r.h.s. of (3.1) the $L_{2}$-norms of $u, \vartheta_{\sigma}, \eta_{\sigma}$ enter only linearly. This is crucial for the proof of global existence (see [12]). The main result of the paper is formulated in Theorem 3.1.

2. Notation and auxiliary results. In Section 3 we use a partition of unity $\left(\left\{\widetilde{\Omega}_{i}\right\},\left\{\zeta_{i}\right\}\right), \Omega \subset \bigcup_{i} \widetilde{\Omega}_{i}$. Let $\widetilde{\Omega}$ be one of the $\widetilde{\Omega}_{i}$ 's and $\zeta(\xi)=\zeta_{i}(\xi)$ be the corresponding function. If $\widetilde{\Omega}$ is an interior subdomain then let $\widetilde{\omega} \subset \widetilde{\Omega}$ and $\zeta(\xi)=1$ for $\xi \in \widetilde{\omega}$. Otherwise we assume that $\overline{\widetilde{\Omega}} \cap S \neq \emptyset, \overline{\widetilde{\omega}} \cap S \neq \emptyset$, $\overline{\widetilde{\omega}} \subset \overline{\widetilde{\Omega}}$. Let $\beta \in \overline{\widetilde{\omega}} \cap S \subset \overline{\widetilde{\Omega}} \cap S, \widetilde{S} \equiv S \cap \widetilde{\Omega}$.

Introduce local coordinates connected with $\{\xi\}$ by

$$
y_{k}=\alpha_{k l}\left(\xi_{l}-\beta_{l}\right), \quad \alpha_{3 k}=n_{k}(\beta), \quad k=1,2,3,
$$

where $\alpha_{k l}$ is a constant orthogonal matrix such that $\widetilde{S}$ is determined by

$$
y_{3}=F\left(y_{1}, y_{2}\right), \quad F \in W_{2}^{5 / 2+\alpha},
$$

and

$$
\widetilde{\Omega}=\left\{y:\left|y_{i}\right|<d, i=1,2, F\left(y^{\prime}\right)<y_{3}<F\left(y^{\prime}\right)+d, y^{\prime}=\left(y_{1}, y_{2}\right)\right\} .
$$

Next, we introduce functions $u^{\prime}, \vartheta^{\prime}$ and $\eta^{\prime}$ by

$$
u_{i}^{\prime}(y)=\left.\alpha_{i j} u_{j}(\xi)\right|_{\xi=\xi(y)}, \quad \vartheta^{\prime}(y)=\left.\vartheta(\xi)\right|_{\xi=\xi(y)}, \quad \eta^{\prime}(y)=\left.\eta(\xi)\right|_{\xi=\xi(y)},
$$

where $\xi=\xi(y)$ is the inverse transformation to (2.1). Further we introduce new variables by

$$
z_{i}=y_{i}, \quad i=1,2, \quad z_{3}=y_{3}-\widetilde{F}(y), \quad y \in \widetilde{\Omega},
$$

which will be denoted by $z=\Phi(y)$, where $\widetilde{F}$ is an extension of $F$ onto $\widetilde{\Omega}$.

Let

$$
\widehat{\Omega}=\Phi(\widetilde{\Omega})=\left\{z:\left|z_{i}\right|<d, i=1,2,0<z_{3}<d\right\}
$$

and $\widehat{S}=\Phi(\widetilde{S})$.

Define

$$
\widehat{f}(z)=\left.f^{\prime}(y)\right|_{y=\Phi^{-1}(z)}, \quad f \in\{u, \vartheta, \eta\} .
$$


Introduce $\widehat{\nabla}_{k}=\left.\xi_{l x_{k}} z_{i \xi_{l}} \nabla_{z_{i}}\right|_{\xi=\chi^{-1}(z)}$, where $\chi(\xi)=\Phi(\psi(\xi))$ and $y=\psi(\xi)$ is defined by (2.1). Introduce also the notation

$$
\begin{array}{ll}
\widetilde{u}(\xi)=u(\xi) \zeta(\xi), & \widetilde{\vartheta}_{\sigma}(\xi)=\vartheta_{\sigma}(\xi) \zeta(\xi), \\
\widetilde{\eta}_{\sigma}(\xi)=\eta_{\sigma}(\xi) \zeta(\xi), & \xi \in \widetilde{\Omega}, \widetilde{\Omega} \cap S=\emptyset, \\
\widetilde{u}(z)=\widehat{u}(z) \widehat{\zeta}(z), & \widetilde{\vartheta}_{\sigma}(z)=\widehat{\vartheta}_{\sigma}(z) \widehat{\zeta}(z), \\
\widetilde{\eta}_{\sigma}(z)=\widehat{\eta}_{\sigma}(z) \widehat{\zeta}(z), & z \in \widehat{\Omega}=\Phi(\widetilde{\Omega}), \widehat{\widetilde{\Omega}} \cap S \neq \emptyset,
\end{array}
$$

where $\widehat{\zeta}(z)=\left.\zeta(\xi)\right|_{\xi=\chi^{-1}(z)}$.

Under the above notation problem (1.6) has the following form in an interior subdomain:

$$
\begin{aligned}
& \eta \widetilde{u}_{i t}-\nabla_{j} T_{i j}\left(\widetilde{u}, \widetilde{p}_{\sigma}\right)=-\nabla_{u_{j}} B_{u i j}(u, \zeta)-T_{u i j}\left(u, p_{\sigma}\right) \nabla_{u_{j}} \zeta \\
& -\left(\nabla_{j} T_{i j}\left(\widetilde{u}, \widetilde{p}_{\sigma}\right)-\nabla_{u_{j}} T_{u i j}\left(\widetilde{u}, \widetilde{p}_{\sigma}\right)\right) \equiv k_{1 i}, \quad i=1,2,3, \\
& \widetilde{\eta}_{\sigma t}+\varrho_{e} \nabla \cdot \widetilde{u}=\varrho_{e} u \cdot \nabla_{u} \zeta-\eta_{\sigma} \nabla_{u} \cdot u \zeta+\varrho_{e}\left(\nabla \cdot \widetilde{u}-\nabla_{u} \cdot \widetilde{u}\right) \equiv k_{2}, \\
& \eta c_{v}(\eta, \vartheta) \widetilde{\vartheta}_{\sigma t}-\varkappa \nabla^{2} \widetilde{\vartheta}_{\sigma}+\theta_{e} p_{\vartheta}\left(\varrho_{e}, \theta_{e}\right) \nabla \cdot \widetilde{u} \\
& =\eta \widetilde{k}+\left[\frac{\mu}{2} \sum_{i, j=1}^{3}\left(\xi_{k x_{i}} \partial_{\xi_{k}} u_{j}+\xi_{k x_{j}} \partial_{\xi_{k}} u_{i}\right)^{2}+(\nu-\mu)\left(\nabla_{u} \cdot u\right)^{2}\right] \zeta \\
& \quad+\theta_{e} p_{\vartheta}\left(\varrho_{e}, \theta_{e}\right) u \cdot \nabla_{u} \zeta+\left(\theta_{e} p_{\vartheta}\left(\varrho_{e}, \theta_{e}\right)-\vartheta p_{\vartheta}(\varrho, \vartheta)\right) \nabla_{u} \cdot u \zeta \\
& \quad+\theta_{e} p_{\vartheta}\left(\varrho_{e}, \theta_{e}\right)\left(\nabla \cdot \widetilde{u}-\nabla_{u} \cdot \widetilde{u}\right)-\varkappa\left(\nabla_{u}^{2} \zeta \vartheta_{\sigma}+2 \nabla_{u} \zeta \cdot \nabla_{u} \vartheta_{\sigma}\right) \\
& \quad-\varkappa\left(\nabla^{2} \widetilde{\vartheta}_{\sigma}-\nabla_{u}^{2} \widetilde{\vartheta}_{\sigma}\right) \equiv \eta \widetilde{k}+k_{3},
\end{aligned}
$$

where

$$
\begin{aligned}
& \mathbb{B}_{u}(u, \zeta)=\left\{B_{u i j}(u, \zeta)\right\}_{i, j=1,2,3} \\
&=\left\{\mu\left(u_{i} \nabla_{u_{i}} \zeta+u_{j} \nabla_{u_{i}} \zeta\right)+(\nu-\mu) \delta_{i j} u \cdot \nabla_{u} \zeta\right\}_{i, j=1,2,3}, \\
& \nabla_{u_{j}}=\xi_{i x_{j}} \partial_{\xi_{i}}, \quad \widetilde{p}_{\sigma}=p_{\sigma} \zeta
\end{aligned}
$$

and in a boundary subdomain:

$$
\begin{gathered}
\widehat{\eta}_{i t}-\nabla_{j} T_{i j}\left(\widetilde{u}, \widetilde{p}_{\sigma}\right)=-\widehat{\nabla}_{j} \widehat{B}_{i j}(\widehat{u}, \widehat{\zeta})-\widehat{T}_{i j}\left(\widehat{u}, \widehat{p}_{\sigma}\right) \widehat{\nabla} \widehat{\nabla}_{j} \\
-\left(\nabla_{j} T_{i j}\left(\widetilde{u}, \widetilde{p}_{\sigma}\right)-\widehat{\nabla}_{j} \widehat{T}_{i j}\left(\widetilde{u}, \widetilde{p}_{\sigma}\right)\right) \equiv k_{4 i}, \quad i=1,2,3, \\
\widetilde{\eta}_{\sigma t}+\varrho_{e} \nabla \cdot \widetilde{u}=\varrho_{e} \widehat{u} \cdot \widehat{\nabla} \widehat{\zeta}-\widehat{\eta}_{\sigma} \widehat{\nabla} \cdot \widehat{u} \widehat{\zeta}+\varrho_{e}(\nabla \cdot \widetilde{u}-\widehat{\nabla} \cdot \widetilde{u}) \equiv k_{5}, \\
\widehat{\eta} c_{v}(\widehat{\eta}, \widehat{\vartheta}) \widetilde{\vartheta}_{\sigma t}-\varkappa \Delta \widetilde{\vartheta}_{\sigma}+\theta_{e} p_{\widehat{\vartheta}}\left(\varrho_{e}, \theta_{e}\right) \nabla \cdot \widetilde{u} \\
=\widehat{\eta} \widetilde{k}+\left[\frac{\mu}{2} \sum_{i, j=1}^{3}\left(\widehat{\nabla}_{i} \widehat{u}_{j}+\widehat{\nabla}_{j} \widehat{u}_{i}\right)^{2}+(\nu-\mu)(\widehat{\nabla} \cdot \widehat{u})^{2}\right] \widehat{\zeta} \\
+\theta_{e} p_{\widehat{\vartheta}}\left(\varrho_{e}, \theta_{e}\right) \widehat{u} \cdot \widehat{\nabla} \widehat{\zeta}+\left(\theta_{e} p_{\widehat{\vartheta}}\left(\varrho_{e}, \theta_{e}\right)-\widehat{\vartheta} p_{\widehat{\vartheta}}(\widehat{\eta}, \widehat{\vartheta})\right) \widehat{\nabla} \cdot \widehat{u} \widehat{\zeta}
\end{gathered}
$$


[cont.]

$$
\begin{aligned}
& +\theta_{e} p_{\widehat{\vartheta}}\left(\varrho_{e}, \theta_{e}\right)(\nabla \cdot \widetilde{u}-\widehat{\nabla} \cdot \widetilde{u})-\varkappa\left(\widehat{\nabla}^{2} \widehat{\zeta} \widehat{\vartheta}_{\sigma}+2 \widehat{\nabla} \widehat{\zeta} \cdot \widehat{\nabla} \widehat{\vartheta}_{\sigma}\right) \\
& -\varkappa\left(\nabla^{2} \widetilde{\vartheta}_{\sigma}-\widehat{\nabla}^{2} \widetilde{\vartheta}_{\sigma}\right) \equiv \widehat{\eta} \widetilde{k}+k_{6}, \\
& \mathbb{T}\left(\widetilde{u}, \widetilde{p}_{\sigma}\right) \widehat{n}^{\prime}=\sigma \Delta_{\widehat{S}} \widehat{\xi} \widehat{\zeta} \cdot \widehat{n} \widehat{n}+\sigma \Delta_{\widehat{S}} \int_{0}^{t} \widetilde{u} d t^{\prime} \cdot \widehat{n} \widehat{n} \\
& +\mathbb{T}\left(\widetilde{u}, \widetilde{p}_{\sigma}\right) \widehat{n}^{\prime}-\widehat{\mathbb{T}}\left(\widetilde{u}, \widetilde{p}_{\sigma}\right) \widehat{n}+\widehat{\mathbb{B}}(\widehat{u}, \widehat{\zeta}) \widehat{n} \\
& -\sigma\left(2 \widehat{\nabla} \int_{0}^{t} \widehat{u} d t^{\prime} \widehat{\nabla} \widehat{\zeta}+\int_{0}^{t} \widehat{u} d t^{\prime} \widehat{\nabla}^{2} \widehat{\zeta}\right) \cdot \widehat{n} \widehat{n}+\frac{2 \sigma}{R_{e}} \widehat{n} \widehat{\zeta} \\
& \equiv \frac{2 \sigma}{R_{e}} \widehat{n} \widehat{\zeta}+k_{7}, \\
& \widehat{n}^{\prime} \cdot \nabla \widetilde{\vartheta}_{\sigma}=\widetilde{\bar{\vartheta}}+\widehat{n} \cdot \widehat{\nabla} \widehat{\zeta}_{\sigma}+\widehat{n}^{\prime} \cdot \nabla \widetilde{\vartheta}_{\sigma}-\widehat{n} \cdot \widehat{\nabla} \widetilde{\vartheta}_{\sigma} \equiv \widetilde{\bar{\vartheta}}+k_{8},
\end{aligned}
$$

where $\widehat{\mathbb{T}}$ and $\widehat{\mathbb{B}}$ indicate that the operator $\nabla_{u}$ is replaced by $\widehat{\nabla} ; \widehat{n}^{\prime}=(0,0,1)$, $\widehat{n}$ is the vector $\bar{n}_{u}=\bar{n}(x(\xi, t), t)$ written in $z$ coordinates and

$$
\Delta_{\widehat{S}}(t)=\frac{1}{\sqrt{g_{\widehat{u}}}} \frac{\partial}{\partial z_{\gamma}}\left(\sqrt{g_{\widehat{u}}} g_{\widehat{u}}^{\gamma \delta} \frac{\partial}{\partial z_{\delta}}\right)
$$

$\left\{g_{\widehat{u}}^{\gamma \delta}\right\}$ is the inverse matrix to $\left\{\left(g_{\widehat{u}}\right)_{\gamma \delta}\right\}$,

$$
\left(g_{\widehat{u}}\right)_{\gamma \delta}=\frac{\partial x}{\partial z_{\gamma}} \cdot \frac{\partial x}{\partial z_{\delta}}, \quad x=\widehat{\xi}+\int_{0}^{t} \widehat{u}\left(z, t^{\prime}\right) d t^{\prime}, \quad g_{\widehat{u}}=\operatorname{det}\left\{\left(g_{\widehat{u}}\right)_{\gamma \delta}\right\} .
$$

From (2.8) we have

$$
\Delta_{\widehat{S}}(0)=\frac{1}{\sqrt{g}} \frac{\partial}{\partial z_{\gamma}}\left(\sqrt{g} g^{\gamma \delta} \frac{\partial}{\partial z_{\delta}}\right),
$$

where by (2.1) and (2.3), $g=\operatorname{det}\left\{g_{\gamma \delta}\right\}=\operatorname{det}\left\{\frac{\partial \xi}{\partial z_{\gamma}} \cdot \frac{\partial \xi}{\partial z_{\delta}}\right\}=1+F_{z_{1}}^{2}+F_{z_{2}}^{2}$ and

$$
g^{11}=g^{-1}\left(1+F_{z_{2}}^{2}\right), \quad g^{22}=g^{-1}\left(1+F_{z_{1}}^{2}\right), \quad g^{12}=g^{21}=-g^{-1} F_{z_{1}} F_{z_{2}} .
$$

We assume that the $d$ from (2.2) and (2.4) is so small that

$$
\left|F_{z_{i}}\right| \leq 1 / 4, \quad i=1,2 .
$$

Now, we introduce the notation and the spaces used in the paper. Let $f=$ $f\left(x_{1}, x_{2}, x_{3}\right)$ be a scalar-valued function defined on a domain $\Omega \subset \mathbb{R}^{3}$. The gradient of $f$ is denoted by $\nabla f$ or $f_{x}$. We also use the notation $\partial_{x} f$. By $f_{x x}$ we denote the matrix $\left\{f_{x_{i} x_{j}}\right\}_{i, j=1,2,3}$ and we set $\left|f_{x x}\right|=\left(\sum_{i, j=1}^{3} f_{x_{i} x_{j}}^{2}\right)^{1 / 2}$.

Next, let $f=f\left(x_{1}, x_{2}, x_{3}\right)$ be a vector-valued function defined on $\Omega \subset$ $\mathbb{R}^{3}$, i.e. $f: \Omega \rightarrow \mathbb{R}^{3}$. By $f_{x}$ we denote the matrix $\left\{f_{i x_{j}}\right\}_{i, j=1,2,3}$. Moreover, 
we use the notation:

$$
f_{x}^{2}=\sum_{i, j=1}^{3} f_{i x_{j}}^{2}, \quad\left|f_{x x}\right|=\left(\sum_{i, j, k=1}^{3} f_{i x_{j} x_{k}}^{2}\right)^{1 / 2} .
$$

Let now $\Omega \subset \mathbb{R}^{3}$ be a bounded domain with boundary $S$ and let $T>0$. By $W_{2}^{k+\alpha, k / 2+\alpha / 2}\left(\Omega^{T}\right), k \in \mathbb{N} \cup\{0\}, \alpha \in(0,1)$, we denote the SobolevSlobodetskiu space with the norm

$$
\begin{aligned}
\|u\|_{W_{2}^{k+\alpha, k / 2+\alpha / 2}\left(\Omega^{T}\right)}= & {\left[\sum_{|\beta|+2 i \leq k}\left\|\partial_{x}^{\beta} \partial_{t}^{i} u\right\|_{L_{2}\left(\Omega^{T}\right)}^{2}\right.} \\
& +\sum_{|\beta|=k} \int_{0}^{T} \int_{\Omega} \int_{\Omega} \frac{\left|\partial_{x}^{\beta} u(x, t)-\partial_{x^{\prime}}^{\beta} u\left(x^{\prime}, t\right)\right|^{2}}{\left|x-x^{\prime}\right|^{3+2 \alpha}} d x d x^{\prime} d t \\
& \left.+\int_{\Omega}^{T} \int_{0}^{T} \frac{\left|\partial_{t}^{[k / 2]} u(x, t)-\partial_{t^{\prime}}^{[k / 2]} u\left(x, t^{\prime}\right)\right|^{2}}{\left|t-t^{\prime}\right|^{1+\alpha+k-2[k / 2]}} d x d t d t^{\prime}\right]^{1 / 2},
\end{aligned}
$$

where $\partial_{x}^{\beta}=\partial_{x_{1}}^{\beta_{1}} \partial_{x_{2}}^{\beta_{2}} \partial_{x_{3}}^{\beta_{3}}, \beta=\left(\beta_{1}, \beta_{2}, \beta_{3}\right)$ is a multi-index, $|\beta|=\beta_{1}+\beta_{2}+\beta_{3}$. Similarly we define the norms in $W_{2}^{k+\alpha}(\Omega)$ and $W_{2}^{k+\alpha, k / 2+\alpha / 2}\left(S^{T}\right)$.

Moreover, we shall use the notation:

$$
\begin{aligned}
& \|u\|_{W_{2}^{k+\alpha, k / 2+\alpha / 2}\left(\Omega^{T}\right)}=\|u\|_{k+\alpha, \Omega^{T}}, \\
& \|u\|_{W_{2}^{k+\alpha}(Q)}=\|u\|_{k+\alpha, Q}, \quad Q \in\left\{\Omega, S, S^{1}\right\}\left(S^{1}\right. \text { is the unit sphere); } \\
& \|u\|_{L_{p}(Q)}=|u|_{p, Q}, \quad \text { where } p \in[1, \infty], Q \in\{\Omega, S\} ; \\
& \|u\|_{L_{2}(Q)}=\|u\|_{0, Q}, \quad \text { where } q \in\left\{\Omega, S, \Omega^{T}, S^{T}\right\} ; \\
& \|u\|_{\Omega^{T}}^{(\alpha+2, \alpha / 2+1)}=\left[\|u\|_{2+\alpha, \Omega^{T}}^{2}+T^{-\alpha}\left(\left\|u_{t}\right\|_{0, \Omega^{T}}^{2}+\sum_{|\beta|=2}\left\|\partial_{x}^{\beta} u\right\|_{0, \Omega^{T}}^{2}\right)\right. \\
& \left.\quad+\sup _{t \leq T}\|u(\cdot, t)\|_{\alpha+1, \Omega}^{2}\right]^{1 / 2} ; \\
& \|u\|_{Q^{T}}^{(\alpha, \alpha / 2)}=\left(\|u\|_{\alpha, Q^{T}}^{2}+T^{-\alpha}\|u\|_{0, Q^{T}}^{2}\right)^{1 / 2}, \quad Q \in\{\Omega, S\} .
\end{aligned}
$$

Next, we define the isotropic Besov spaces by introducing the norm (see $[3$, Sect. 18])

$$
\|u\|_{B_{p}^{l}\left(\mathbb{R}^{n}\right)}=\|u\|_{L_{p}\left(\mathbb{R}^{n}\right)}+\sum_{i=1}^{n}\left(\int_{0}^{h_{0}} d h \int_{\mathbb{R}^{n}} d x \frac{\left|\Delta_{i}^{m}(h) \partial_{x_{i}}^{k} u\right|^{p}}{h^{1+(l-k) p}}\right)^{1 / p}
$$

where $p \in[1, \infty]$,

$$
\Delta_{i}^{m}(h) f(x)=\sum_{j=0}^{m}(-1)^{m-j} c_{j m} f\left(x+j h e_{i}\right),
$$


$c_{j m}=\left(\begin{array}{c}m \\ j\end{array}\right)=m ! /(j !(m-j) !), x \in \mathbb{R}^{n}, e_{i}$ is the $i$ th unit vector, $i=1, \ldots, n$, and $m>l-k, m, k \in \mathbb{N} \cup\{0\}, l \in \mathbb{R}_{+}, l \notin \mathbb{Z}$.

It is proved in [4] that for all $m, k$ satisfying $m>l-k$ the norms defined by (2.10) are equivalent.

Now, we define the Sobolev-Slobodetskiu spaces by introducing the norm

$$
\|u\|_{W_{p}^{l}\left(\mathbb{R}^{n}\right)}=\|u\|_{L_{p}\left(\mathbb{R}^{n}\right)}+\sum_{i=1}^{n}\left(\int_{0}^{h_{0}} d h \int_{\mathbb{R}^{n}} d x \frac{\left|\Delta_{i}(h) \partial_{x_{i}}^{[l]} u\right|^{p}}{h^{1+p(l-[l])}}\right)^{1 / p}
$$

where $\Delta_{i}(h)=\Delta_{i}^{1}(h), l \notin \mathbb{Z},[l]$ is the integer part of $l$. alent.

By the Golovkin theorem [4] the norms of $B_{p}^{l}\left(\mathbb{R}^{n}\right)$ and $W_{p}^{l}\left(\mathbb{R}^{n}\right)$ are equiv-

We also define the spaces $\widetilde{B}_{p}^{l}\left(\mathbb{R}^{n}\right)$ and $\widetilde{W}_{p}^{l}\left(\mathbb{R}^{n}\right)$ by introducing the norms

$$
\|u\|_{\widetilde{B}_{p}^{l}\left(\mathbb{R}^{n}\right)}=\|u\|_{L_{p}\left(\mathbb{R}^{n}\right)}+\left(\int_{\mathbb{R}^{n}} d x \int_{\mathbb{R}^{n}} d y \frac{\left|\Delta^{m}(x-y) \partial_{y}^{k} u(y)\right|^{p}}{|x-y|^{n+p(l-k)}}\right)^{1 / p}
$$

and

$$
\|u\|_{\widetilde{W}_{p}^{l}\left(\mathbb{R}^{n}\right)}=\|u\|_{L_{p}\left(\mathbb{R}^{n}\right)}+\left(\int_{\mathbb{R}^{n}} d x \int_{\mathbb{R}^{n}} d y \frac{\left|\Delta(x-y) \partial_{y}^{[l]} u(y)\right|^{p}}{|x-y|^{n+p(l-[l])}}\right)^{1 / p},
$$

where $\Delta^{m}(x-y) f(y)=\sum_{i=1}^{m} \Delta_{i}^{m}(h) f(y),(x-y) \cdot e_{i}=h, \Delta(x-y) f(y)=$ $\Delta^{1}(x-y) f(y)$ and $m>l-k, m, k \in \mathbb{N} \cup\{0\}, l \in \mathbb{R}_{+}, l \notin \mathbb{Z}$.

By Lemma 7.44 of [1] the norms of $\widetilde{B}_{p}^{l}\left(\mathbb{R}^{n}\right), B_{p}^{l}\left(\mathbb{R}^{n}\right)\left(\right.$ with $\left.h_{0}=\infty\right)$, $\widetilde{W}_{p}^{l}\left(\mathbb{R}^{n}\right)$ and $W_{p}^{l}\left(\mathbb{R}^{n}\right)$ (with $h_{0}=\infty$ ) are equivalent.

Now, we recall the following imbedding for the Besov spaces (2.10) (see [3, Sect. 18]):

$$
\partial_{x}^{\sigma} B_{p}^{l}\left(\mathbb{R}^{n}\right) \subset B_{q}^{\varrho}\left(\mathbb{R}^{n}\right) \quad \text { for } n / p-n / q+|\sigma|+\varrho \leq l .
$$

Moreover, for

$$
\varkappa=\frac{1}{l}\left(\frac{n}{p}-\frac{n}{q}+|\sigma|+\varrho\right)>0 \quad \text { and } \quad \varkappa_{1}=\frac{3}{p}-\frac{3}{r}
$$

we have the interpolation inequality

$$
\left\|\partial_{x}^{\sigma} u\right\|_{B_{q}^{o}\left(\mathbb{R}^{n}\right)} \leq\left(\varepsilon^{1-\varkappa}+c \varepsilon^{-\varkappa} \varepsilon_{1}^{1-\varkappa_{1}}\right)\|u\|_{B_{p}^{l}\left(\mathbb{R}^{n}\right)}+c \varepsilon^{-\varkappa} \varepsilon_{1}^{-\varkappa_{1}}\|u\|_{L_{r}\left(\mathbb{R}^{n}\right)}
$$

with $r \geq 1$.

In the above notation $B_{p}^{l}\left(\mathbb{R}^{n}\right)$ with $l \in \mathbb{Z}_{+}$is the Sobolev space.

By $C_{B}^{k}(Q)\left(Q \subset \mathbb{R}^{n}\right.$ is a domain) we denote the space of functions $u \in C^{k}(Q)$ such that $D^{\sigma} u, 0 \leq|\sigma| \leq k$, is bounded on $Q$, with the norm $\|u\|_{C_{B}^{k}(Q)}=\max _{0 \leq|\sigma| \leq k} \sup _{x \in Q}\left|D^{\sigma} u(x)\right|$. 
All the above remarks apply to spaces of functions defined on a bounded domain $\Omega \subset \mathbb{R}^{n}$, and by using a partition of unity we can also define spaces of traces on the boundary of $\Omega$ and formulate the corresponding trace theorems.

3. Inequality for the global existence. In order to derive global estimates we assume the following condition: $\Omega_{t}$ is diffeomorphic to a ball and $S_{t}$ can be described by

$$
|x|=r=R(\omega, t), \quad \omega \in S^{1} .
$$

The main result of the paper is the following theorem.

TheOrem 3.1. Let $k \in W_{2}^{\alpha, \alpha / 2}\left(\Omega^{T}\right), \bar{\vartheta} \in W_{2}^{\alpha+1 / 2, \alpha / 2+1 / 4}\left(S^{T}\right), \alpha \in$ $(3 / 4,1)$ and assume that $(u, \vartheta, \eta)$ is the local solution of problem (1.6) determined by Theorem 3.4 of [13]. Moreover, let $\rho^{*}=\sup _{\Omega^{T}} \eta, \rho^{*}=\inf _{\Omega^{T}} \eta$, $\theta^{*}=\sup _{\Omega^{T}} \vartheta, \theta^{*}=\inf _{\Omega^{T}} \vartheta, 0<\rho_{*}<\rho^{*}<\infty, 0<\theta_{*}<\theta^{*}<\infty$. Then

$$
\begin{aligned}
& \phi(t, \Omega)+\bar{c}\left(\|u\|_{2+\alpha, \Omega^{t}}^{2}+\left\|\vartheta_{\sigma}\right\|_{2+\alpha, \Omega^{t}}^{2}+\left\|\eta_{\sigma}\right\|_{1+\alpha, \Omega^{t}}^{2}\right) \\
\leq & c\left(t, Z_{1}, Z_{2}\right)\left[\|u\|_{0, \Omega^{t}}^{2}+\left\|\eta_{\sigma}\right\|_{0, \Omega^{t}}^{2}+\left\|\vartheta_{\sigma}\right\|_{0, \Omega^{t}}^{2}+\|k\|_{\alpha, \Omega^{t}}^{2}+\|\bar{\vartheta}\|_{\alpha+1 / 2, S^{t}}^{2}\right. \\
& \left.+\varepsilon_{1} Z_{4}+Z_{1} Z_{3}\right]+\phi(0, \Omega),
\end{aligned}
$$

where $t \leq T, T$ is the time of local existence; $\phi(t, \Omega)$ is defined by (3.86); $c=c\left(t, Z_{1}, Z_{2}\right)$ is a positive continuous function nondecreasing with respect to its arguments; $\bar{c} \leq 1$ is a positive constant; $\varepsilon_{1} \in(0,1)$ is a sufficiently small constant and

$$
\begin{aligned}
& Z_{1}=\|u\|_{2+\alpha, \Omega^{t}}^{2}+\left\|\vartheta_{\sigma}\right\|_{2+\alpha, \Omega^{t}}^{2}+\left\|\eta_{\sigma}\right\|_{1+\alpha, \Omega^{t}}^{2}, \\
& Z_{2}=\sup _{0 \leq t^{\prime} \leq t}\|u\|_{1+\alpha, \Omega}^{2}+\sup _{0 \leq t^{\prime} \leq t}\left\|\vartheta_{\sigma}\right\|_{1+\alpha, \Omega}^{2}+\sup _{0 \leq t^{\prime} \leq t}\left\|\eta_{\sigma}\right\|_{1+\alpha, \Omega}^{2}, \\
& Z_{3}=\left[\|u\|_{\Omega^{t}}^{(\alpha+2, \alpha / 2+1)}+\left\|\vartheta_{\sigma}\right\|_{\Omega^{t}}^{(\alpha+2, \alpha / 2+1)}\right]^{2}+\left\|\eta_{\sigma}\right\|_{1+\alpha, \Omega^{t}}^{2}+\sup _{0 \leq t^{\prime} \leq t}\left\|\eta_{\sigma}\right\|_{1+\alpha, \Omega}^{2}, \\
& Z_{4}=t\left\|H(\cdot, 0)+2 / R_{e}\right\|_{\alpha+1 / 2, S}^{2}+\int_{0}^{t}\left\|R\left(\cdot, t^{\prime}\right)-R(\cdot, 0)\right\|_{5 / 2+\alpha, S^{1}}^{2} d t^{\prime} .
\end{aligned}
$$

Inequality (3.1) is derived in several steps. First, we consider problem (2.6) to obtain an inequality similar to (3.1) in an interior subdomain $\widetilde{\Omega}$. In order to get this inequality one has to estimate separately the norms $\int_{0}^{t}\|\widetilde{u}\|_{2+\alpha, \widetilde{\Omega}}^{2} d t^{\prime}, \int_{0}^{t}\left\|\widetilde{\theta}_{\sigma}\right\|_{2+\alpha, \widetilde{\Omega}}^{2} d t^{\prime}, \int_{0}^{t}\left\|\widetilde{\eta}_{\sigma}\right\|_{1+\alpha, \widetilde{\Omega}}^{2} d t^{\prime}$ and the seminorms $\int_{\widetilde{\Omega}}\left\|\widetilde{u}_{t^{\prime}}\right\|_{\alpha / 2,(0, t)}^{2} d \xi, \int_{\widetilde{\Omega}}\left\|\widetilde{\vartheta}_{\sigma t^{\prime}}\right\|_{\alpha / 2,(0, t)}^{2} d \xi$.

The resulting inequality (see (3.37)) contains terms similar to (3.1) except for the seminorm $\int_{\widetilde{\Omega}}\left\|\eta_{\sigma t^{\prime}}\right\|_{\alpha / 2,(0, t)}^{2} d \xi$ on the left-hand side. 
Next, we consider problem (2.7) in order to derive an inequality in a boundary subdomain $\widehat{\Omega}$. Now, to estimate the norms $\int_{0}^{t}\|\widetilde{u}\|_{2+\alpha, \widehat{\Omega}}^{2} d t^{\prime}$, $\int_{0}^{t}\left\|\widetilde{\vartheta}_{\sigma}\right\|_{2+\alpha, \widehat{\Omega}}^{2} d t^{\prime}$ and the seminorms $\int_{\widehat{\Omega}}\left\|\widetilde{u}_{t^{\prime}}\right\|_{\alpha / 2,(0, t)} d z, \int_{\widehat{\Omega}}\left\|\widetilde{\vartheta}_{\sigma t^{\prime}}\right\|_{\alpha / 2,0, t)}^{2} d z$ the boundary conditions $(2.7)_{4}-(2.7)_{5}$ have to be taken into account. First we get estimates of the derivatives of $\widetilde{u}$ and $\widetilde{\vartheta}_{\sigma}$ in the directions tangent to $\widehat{S}$ and then using these estimates we are able to estimate the derivatives of $\widetilde{u}$ and $\widetilde{\vartheta}_{\sigma}$ in the normal direction to $\widehat{S}$. Finally, by using equation $(1.6)_{2}$ we obtain an estimate of $\int_{\Omega}\left\|\eta_{\sigma t^{\prime}}\right\|_{\alpha / 2,(0, t)}^{2} d \xi$.

It should be underlined that the form of inequality (3.1) is precisely connected with the method of proof of the global existence theorem for problem (1.1), presented in [12]. Therefore, in the long process of obtaining the successive estimates it is essential to control their right-hand sides in order to obtain forms similar to (3.1).

Since the proof of Theorem 3.1 is very long, its consecutive steps will be presented in separate lemmas.

First, we will obtain estimates which hold in interior subdomains $\widetilde{\Omega}_{i}$ (see Section 2).

Let us consider an interior subdomain $\widetilde{\Omega}$ and let $\zeta \in C_{0}^{\infty}(\widetilde{\Omega})$ be the corresponding function from the partition of unity. We can assume that

$$
\widetilde{\Omega}=B_{2 \lambda}\left(\xi_{0}\right)=\left\{\zeta \in \mathbb{R}^{3}:\left|\xi-\xi_{0}\right|<2 \lambda\right\}
$$

and that $\zeta(\xi)=1$ in $B_{\lambda+\varepsilon_{0}}\left(\xi_{0}\right)=\left\{\xi \in \mathbb{R}^{3}:\left|\xi-\xi_{0}\right|<\lambda+\varepsilon_{0}\right\}$, for some $-\lambda<\varepsilon_{0}<\lambda$, while $\zeta(\xi)=0$ for $\xi \in \mathbb{R}^{3} \backslash B_{2 \lambda}\left(\xi_{0}\right)$.

Denote by $\Delta^{s}(z) f(\xi)$ the $s$ th finite difference of $f$ such that

$$
\Delta^{s}(z) f(\xi)=\sum_{k=0}^{s}\left(\begin{array}{l}
s \\
k
\end{array}\right)(-1)^{s-k} f(\xi+k z) .
$$

We start with the following lemma.

Lemma 3.2. Let the assumptions of Theorem 3.1 be satisfied. Then for the functions $\widetilde{u}, \widetilde{\eta}_{\sigma}, \widetilde{\vartheta}_{\sigma}$ defined by (2.5) the following estimate holds:

$$
\begin{aligned}
\frac{1}{2} \frac{d}{d t} \int_{\mathbb{R}^{3}} d z & \int_{B_{2 \lambda}\left(\xi_{0}\right)} d \xi\left(\frac{\eta\left|\Delta^{2}(z) \widetilde{u}\right|^{2}}{|z|^{3+2(1+\alpha)}}+\frac{p_{1}\left|\Delta^{2}(z) \widetilde{\eta}_{\sigma}\right|^{2}}{\varrho_{e}|z|^{3+2(1+\alpha)}}\right. \\
& \left.+\frac{p_{2} \eta c_{v}}{\theta_{e} p_{\vartheta}\left(\varrho_{e}, \theta_{e}\right)} \frac{\left|\Delta^{2}(z) \widetilde{\vartheta}_{\sigma}\right|^{2}}{|z|^{3+2(1+\alpha)}}\right)+c_{1} \int_{\mathbb{R}^{3}} d z \frac{\left\|\Delta^{2}(z) \widetilde{u}\right\|_{1, B_{2 \lambda}\left(\xi_{0}\right)}^{2}}{|z|^{3+2(1+\alpha)}} \\
& +\varkappa \int_{\mathbb{R}^{3}} d z \int_{B_{2 \lambda}\left(\xi_{0}\right)} d \xi \frac{p_{2}\left|\nabla\left(\Delta^{2}(z) \widetilde{\vartheta}_{\sigma}\right)\right|^{2}}{|z|^{3+2(1+\alpha)}}
\end{aligned}
$$




$$
\begin{aligned}
\leq & \varepsilon\left(\|u\|_{2+\alpha, \widetilde{\Omega}}^{2}+\left\|\eta_{\sigma}\right\|_{1+\alpha, \widetilde{\Omega}}^{2}+\left\|\vartheta_{\sigma}\right\|_{2+\alpha, \widetilde{\Omega}}^{2}\right) \\
& +\psi_{14}(1 / \varepsilon, a)\left[X_{1} X_{2}+X_{1}\left(1+X_{2}^{2}\right) \int_{0}^{t}\|u\|_{2+\alpha, \Omega}^{2} d t^{\prime}+\left\|\eta_{\sigma}\right\|_{1+\alpha, \Omega}^{2}\left\|\widetilde{u}_{t}\right\|_{\alpha, \widetilde{\Omega}}^{2}\right] \\
& +\psi_{15}(1 / \varepsilon, a)\left(\|u\|_{1+\alpha, \widetilde{\Omega}}^{2}+\left\|\widetilde{\eta}_{\sigma}\right\|_{\alpha, \widetilde{\Omega}}^{2}+\left\|\widetilde{\vartheta}_{\sigma}\right\|_{\alpha, \widetilde{\Omega}}^{2}+\left\|\vartheta_{\sigma}\right\|_{1+\alpha, \widetilde{\Omega}}^{2}\right) \\
& +\psi_{16}(1 / \varepsilon, a)\|\widetilde{k}\|_{\alpha, \widetilde{\Omega}}^{2},
\end{aligned}
$$

where $t \leq T$, and

$$
\begin{aligned}
& X_{1}=\|u\|_{2+\alpha, \Omega}^{2}+\left\|\eta_{\sigma}\right\|_{1+\alpha, \Omega}^{2}+\left\|\vartheta_{\sigma}\right\|_{2+\alpha, \Omega}^{2}, \\
& X_{2}=\|u\|_{1+\alpha, \Omega}^{2}+\left\|\eta_{\sigma}\right\|_{1+\alpha, \Omega}^{2}+\left\|\vartheta_{\sigma}\right\|_{1+\alpha, \Omega}^{2} .
\end{aligned}
$$

Proof. Similarly to [5] we apply $\Delta^{2}(z)$ to $(2.6)_{1}-(2.6)_{3}$ and multiply the resulting equations by $\Delta^{2}(z) \widetilde{u}_{i}, \frac{p_{1}}{\varrho_{e}} \Delta^{2}(z) \widetilde{\eta}_{\sigma}$ and $\frac{p_{2}}{\theta_{e} p_{\vartheta}\left(\varrho_{e}, \theta_{e}\right)} \Delta^{2}(z) \widetilde{\vartheta}_{\sigma}$, respectively. Then integrating each of the equations over $B_{2 \lambda}\left(\xi_{0}\right)$ and with respect to $z$ over $\mathbb{R}^{3}$ with the weight $1 /|z|^{3+2(\alpha+1)}$ and using (1.5), (1.6) 2 and the Korn inequality we obtain

$$
\begin{aligned}
& \frac{1}{2} \frac{d}{d t} \int_{\mathbb{R}^{3}} d z \int_{B_{2 \lambda}\left(\xi_{0}\right)} d \xi\left(\frac{\eta\left|\Delta^{2}(z) \widetilde{u}\right|^{2}}{|z|^{3+2(1+\alpha)}}+\frac{p_{1}\left|\Delta^{2}(z) \widetilde{\eta}_{\sigma}\right|^{2}}{\varrho_{e}|z|^{3+2(1+\alpha)}}\right. \\
& \left.\quad+\frac{p_{2} \eta c_{v}}{\theta_{e} p_{\vartheta}\left(\varrho_{e}, \theta_{e}\right)} \frac{\left|\Delta^{2}(z) \widetilde{\vartheta}_{\sigma}\right|^{2}}{|z|^{3+2(1+\alpha)}}\right) \\
& \quad+c_{1} \int_{\mathbb{R}^{3}} d z \frac{\left\|\Delta^{2}(z) \widetilde{u}\right\|_{1, B_{2 \lambda}\left(\xi_{0}\right)}^{2}}{|z|^{3+2(1+\alpha)}}+\varkappa \int_{\mathbb{R}^{3}} d z \int_{B_{2 \lambda}\left(\xi_{0}\right)} d \xi \frac{p_{2}\left|\Delta^{2}(z) \nabla \widetilde{\vartheta}_{\sigma}\right|^{2}}{|z|^{3+2(1+\alpha)}} \\
& \leq I_{1}+\int_{\mathbb{R}^{3}} d z \int_{B_{2 \lambda}\left(\xi_{0}\right)} d \xi \frac{p_{2} \Delta^{2}(z) k_{3} \Delta^{2}(z) \widetilde{\vartheta}_{\sigma}}{|z|^{3+2(1+\alpha)}}
\end{aligned}
$$

where $c_{1}>0$ is a constant and

$$
\begin{aligned}
I_{1} \leq & \varepsilon\left(\|\widetilde{u}\|_{2+\alpha, \widetilde{\Omega}}^{2}+\left\|\vartheta_{\sigma}\right\|_{2+\alpha, \widetilde{\Omega}}^{2}+\int_{\mathbb{R}^{3}} d z \int_{B_{2 \lambda}\left(\xi_{0}\right)} d \xi \frac{\left|\Delta^{2}(z) \widetilde{\eta}_{\sigma}\right|^{2}}{|z|^{3+2(1+\alpha)}}\right) \\
& +\psi_{1}(1 / \varepsilon, a)\left(\|\widetilde{u}\|_{1+\alpha, \widetilde{\Omega}}^{2}+\left\|\eta_{\sigma}\right\|_{\alpha, \widetilde{\Omega}}^{2}+\left\|\vartheta_{\sigma}\right\|_{\alpha, \widetilde{\Omega}}^{2}\right) \\
& +\psi_{2}(1 / \varepsilon, a)\left[\int _ { 0 } ^ { t } \| u \| _ { 2 + \alpha , \Omega } ^ { 2 } d t ^ { \prime } \left(\|\widetilde{u}\|_{2+\alpha, \widetilde{\Omega}}^{2}+\left\|\eta_{\sigma}\right\|_{1+\alpha, \widetilde{\Omega}}^{2}\right.\right. \\
& \left.+\left\|\vartheta_{\sigma}\right\|_{1+\alpha, \widetilde{\Omega}}^{2}+\left\|\widetilde{\eta}_{\sigma}\right\|_{1+\alpha, \widetilde{\Omega}}^{2}\|\widetilde{u}\|_{2+\alpha, \widetilde{\Omega}}^{2}+\left\|\widetilde{\vartheta}_{\sigma}\right\|_{1+\alpha, \widetilde{\Omega}}^{2}\|\widetilde{u}\|_{2+\alpha, \widetilde{\Omega}}^{2}\right) \\
& +\|u\|_{2+\alpha, \Omega}^{2}\|\widetilde{u}\|_{2+\alpha, \widetilde{\Omega}}^{2}+\left\|\eta_{\sigma}\right\|_{1+\alpha, \Omega}^{2}\left\|\widetilde{u}_{t}\right\|_{\alpha, \widetilde{\Omega}}^{2}
\end{aligned}
$$




$$
\begin{aligned}
& +\left(\left\|\widetilde{\eta}_{\sigma}\right\|_{1+\alpha, \widetilde{\Omega}}^{2}+\|\widetilde{u}\|_{2+\alpha, \widetilde{\Omega}}^{2}+\left\|\widetilde{\vartheta}_{\sigma}\right\|_{1+\alpha, \widetilde{\Omega}}^{2}\right)\left(\left\|\eta_{\sigma}\right\|_{1+\alpha, \Omega}^{2}+\left\|\vartheta_{\sigma}\right\|_{1+\alpha, \Omega}^{2}\right) \\
& \left.+\left(\left\|\vartheta_{\sigma t}\right\|_{1+\alpha, \Omega}^{2}+\left\|\vartheta_{\sigma}\right\|_{2+\alpha, \Omega}^{2}\right)\left\|\widetilde{\vartheta}_{\sigma}\right\|_{1+\alpha, \widetilde{\Omega}}^{2}+\left\|\eta_{\sigma}\right\|_{1+\alpha, \Omega}^{2}\|\tilde{k}\|_{\alpha, \widetilde{\Omega}}^{2}\right]
\end{aligned}
$$

where $a=t^{1 / 2}\left(\int_{0}^{t}\|u\|_{2+\alpha, \Omega}^{2} d t^{\prime}\right)^{1 / 2}$, and $\psi_{i}(i=1,2)$ and all functions $\psi_{i}$ occurring below are positive continuous nondecreasing functions of their arguments.

Next, we obtain

$$
\int_{\mathbb{R}^{3}} d z \int_{B_{2 \lambda}\left(\xi_{0}\right)} d \xi \frac{p_{2} \Delta^{2}(z) k_{3} \Delta^{2}(z) \tilde{\vartheta}_{\sigma}}{|z|^{3+2(1+\alpha)}}
$$$$
=-\int_{B_{2 \lambda}(0)} d z \int_{B_{2 \lambda}\left(\xi_{0}\right)} d \xi \frac{\Delta(z) k_{3} \Delta(z) p_{2} \Delta^{2}(z) \tilde{\vartheta}_{\sigma}}{|z|^{3+2(1+\alpha)}}
$$$$
-\int_{B_{2 \lambda}(0)} d z \int_{B_{2 \lambda}\left(\xi_{0}\right)} d \xi \frac{p_{2} \Delta(z) k_{3} \Delta^{3}(z) \tilde{\vartheta}_{\sigma}}{|z|^{3+2(1+\alpha)}}
$$

$\leq \varepsilon \int_{B_{2 \lambda}(0)} d z \int_{B_{2 \lambda}\left(\xi_{0}\right)} d \xi \frac{\left|\Delta^{3}(z) \tilde{\vartheta}_{\sigma}\right|^{2}}{|z|^{3+2(2+\alpha)}}+c(\varepsilon) \int_{B_{2 \lambda}(0)} d z \int_{B_{2 \lambda}\left(\xi_{0}\right)} d \xi \frac{\left|\Delta(z) k_{3}\right|^{2}}{|z|^{3+2 \alpha}}$

$+c\left[\int_{B_{2 \lambda}(0)} d z \int_{B_{2 \lambda}\left(\xi_{0}\right)} d \xi \frac{\left|\Delta(z) \eta_{\sigma}\right|^{p_{1}}}{|z|^{3+\frac{p_{1}}{2}(1+\alpha)}}+\int_{B_{2 \lambda}(0)} d z \int_{B_{2 \lambda}\left(\xi_{0}\right)} d \xi \frac{\left|\Delta(z) \vartheta_{\sigma}\right|^{p_{1}}}{|z|^{3+\frac{p_{1}}{2}(1+\alpha)}}\right]^{1 / p_{1}}$

$\cdot\left(\int_{B_{2 \lambda}(0)} d z \int_{B_{2 \lambda}\left(\xi_{0}\right)} d \xi \frac{\left|\Delta(z) k_{3}\right|^{p_{2}}}{|z|^{3+\frac{p_{2}}{2}(1+\alpha)}}\right)^{1 / p_{2}}$

$\cdot\left(\int_{B_{2 \lambda}(0)} d z \int_{B_{2 \lambda}\left(\xi_{0}\right)} d \xi \frac{\left|\Delta^{2}(z) \widetilde{\vartheta}_{\sigma}\right|^{p_{3}}}{|z|^{3+p_{3}(1+\alpha)}}\right)^{1 / p_{3}} \leq \varepsilon\left\|\vartheta_{\sigma}\right\|_{2+\alpha, \widetilde{\Omega}}^{2}+I_{2}+I_{3}$

with

$$
\begin{aligned}
\left|I_{2}\right| \leq & c \int_{B_{2 \lambda}(0)} d z \int_{B_{2 \lambda}\left(\xi_{0}\right)} d \xi \frac{1}{|z|^{3+2 \alpha}}\left(\left|\Delta(z) \xi_{x}^{2}\right|^{2}\left|u_{\xi}^{2}\right|^{2}+\left|\Delta(z) u \cdot \nabla_{u} \zeta\right|^{2}\right. \\
& +\left|\Delta(z)\left(\theta_{e} p_{\vartheta}\left(\varrho_{e}, \theta_{e}\right)-\vartheta p_{\vartheta}(\eta, \vartheta)\right) \nabla_{u} \cdot u \zeta\right|^{2} \\
& +\left|\Delta(z)\left(\nabla \cdot \widetilde{u}-\nabla_{u} \cdot \widetilde{u}\right)\right|^{2} \\
& +\left|\Delta(z)\left(\nabla_{u}^{2} \zeta \vartheta_{\sigma}+2 \nabla_{u} \zeta \cdot \nabla_{u} \vartheta_{\sigma}\right)\right|^{2} \\
& \left.+\left|\Delta(z)\left(\nabla^{2} \widetilde{\vartheta}_{\sigma}-\nabla_{u}^{2} \widetilde{\vartheta}_{\sigma}\right)\right|^{2}\right) \equiv \sum_{i=1}^{6} I_{2}^{i}
\end{aligned}
$$


and

$$
\left|I_{3}\right| \leq \varepsilon\left\|\vartheta_{\sigma}\right\|_{2+\alpha, \widetilde{\Omega}}^{2}+c(\varepsilon)\left[\left\|k_{3}\right\|_{\alpha, \widetilde{\Omega}}^{2}\left(\left\|\eta_{\sigma}\right\|_{1+\alpha, \widetilde{\Omega}}^{2}+\left\|\vartheta_{\sigma}\right\|_{1+\alpha, \widetilde{\Omega}}^{2}\right)\right],
$$

where $1 / p_{1}+1 / p_{2}+1 / p_{3}=1$ and we have used the imbeddings $B_{2}^{1+\alpha}(\Omega) \subset$ $B_{p_{1}}^{(1+\alpha) / 2}(\Omega), B_{2}^{\alpha}(\Omega) \subset B_{p_{2}}^{(1+\alpha) / 2}(\Omega), B_{2}^{2+\alpha}(\Omega) \subset B_{p_{3}}^{1+\alpha}(\Omega)$ valid for $3 / 2-$ $3 / p_{1}+1+\alpha / 2 \leq 1+\alpha, 3 / 2-3 / p_{2}+1+\alpha / 2 \leq \alpha, 3 / 2-3 / p_{3}+1+\alpha \leq 2+\alpha$, which all hold for suitable $p_{i}$ if $\alpha>1 / 2$.

Now, we estimate the terms on the right-hand side of (3.8). We have

$$
\begin{aligned}
I_{2}^{1} \leq & c \int_{B_{2 \lambda}(0)} d z \int_{B_{2 \lambda}\left(\xi_{0}\right)} d \xi \frac{\left|\Delta(z) u_{\xi}\right|^{2}\left|u_{\xi}\right|^{2}}{|z|^{3+2 \alpha}} \\
& +c \int_{B_{2 \lambda}(0)} d z \int_{B_{2 \lambda}\left(\xi_{0}\right)} d \xi \frac{\left|\Delta(z) \int_{0}^{t} u_{\xi} d t^{\prime}\right|^{2}\left|\psi_{3}(b) u_{\xi}^{2}\right|^{2}}{|z|^{3+2 \alpha}} \\
& +c \int_{B_{2 \lambda}(0)} d z \int_{B_{2 \lambda}\left(\xi_{0}\right)} d \xi \frac{\left|\int_{0}^{t} u_{\xi} d t^{\prime}\right|^{2}\left|\Delta(z) \psi_{3}(b) u_{\xi}^{2}\right|^{2}}{|z|^{3+2 \alpha}} \\
& +c \int_{B_{2 \lambda}(0)} d z \int_{B_{2 \lambda}\left(\xi_{0}\right)} d \xi \frac{\left|\Delta(z) \int_{0}^{t} u_{\xi} d t^{\prime}\right|^{2}\left|\psi_{3}(b) u_{\xi}^{2}\right|^{2}}{|z|^{3+2 \alpha}} \\
\leq & c\left|u_{\xi}\right|_{\infty, \Omega}^{2} \int_{\mathbb{R}^{3}} d z \int_{B_{2 \lambda}\left(\xi_{0}\right)} d \xi \frac{\left|\Delta(z) u_{\xi}\right|^{2}}{|z|^{3+2 \alpha}} \\
& +c \psi_{4}(a)\left|u_{\xi}\right|_{\infty, \Omega}^{2}\left[\left(\int_{B_{2 \lambda}(0)} d z \int_{B_{2 \lambda}\left(\xi_{0}\right)} d \xi \frac{\left|u_{\xi}\right|^{2 p}}{|z|^{3-\delta p}}\right)^{1 /(2 p)}\right]^{2} \\
& \times\left[\left(\int_{\mathbb{R}^{3}} d z \int_{B_{2 \lambda}\left(\xi_{0}\right)} d \xi \frac{\left|\Delta(z) \int_{0}^{t} u_{\xi} d t^{\prime}\right|^{2 p^{\prime}}}{|z|^{3+2 p^{\prime} \alpha+\delta p^{\prime}}}\right)^{1 /\left(2 p^{\prime}\right)}\right]^{2} \\
& +c \psi_{5}(a)\left|\int_{0}^{t} u_{\xi}\left(\xi, t^{\prime}\right) d t^{\prime}\right|_{\infty, \Omega}^{2}\left|u_{\xi}\right|_{\infty, \Omega}^{2} \int_{\mathbb{R}^{3}} d z B_{B_{2 \lambda}\left(\xi_{0}\right)} d \xi \frac{\left|\Delta(z) u_{\xi}\right|^{2}}{|z|^{3+2 \alpha}}
\end{aligned}
$$

where $\delta>0$ is sufficiently small and $1 / p+1 / p^{\prime}=1, b=\int_{0}^{t} u_{\xi} d t^{\prime}$ and we have used the fact that $\xi_{x}=I+b \psi_{3}(b), I$ is the unit matrix and $\psi_{3}(0) \neq 0$, $\psi_{3}$ is a positive continuous increasing function.

Using the imbeddings $B_{2}^{\alpha}(\Omega) \subset L_{2 p}(\Omega)$ and $B_{2}^{1+\alpha}(\Omega) \subset B_{2 p^{\prime}}^{\alpha+\delta / 2}(\Omega)$ which hold simultaneously for $\alpha>1 / 2$ and some $\delta, p, p^{\prime}$ such that $1 / p+$ $1 / p^{\prime}=1,3 / 2-3 /(2 p) \leq \alpha$ and $3 / 2-3 /\left(2 p^{\prime}\right)+\alpha+\delta / 2 \leq 1+\alpha$ (see imbedding (2.11)) we get 
(3.11) $\quad I_{2}^{1} \leq c\|u\|_{2+\alpha, \Omega}^{2}\|u\|_{1+\alpha, \Omega}^{2}+\psi_{6}(a)\|u\|_{2+\alpha, \Omega}^{2}\|u\|_{1+\alpha, \Omega}^{2} \int_{0}^{t}\|u\|_{2+\alpha, \Omega}^{2} d t^{\prime}$.

In the same way we obtain

$$
\begin{aligned}
I_{2}^{2}+I_{2}^{5} \leq & c\left(\|u\|_{\alpha, \Omega}^{2}+\left\|\vartheta_{\sigma}\right\|_{1+\alpha, \Omega}^{2}\right) \\
& +\psi_{7}(a) \int_{0}^{t}\|u\|_{2+\alpha, \Omega}^{2} d t^{\prime}\left(\|u\|_{1+\alpha, \Omega}^{2}+\left\|\vartheta_{\sigma}\right\|_{2+\alpha, \Omega}^{2}\right) .
\end{aligned}
$$

Next, we estimate

$$
\begin{aligned}
I_{2}^{6} \leq & c \int_{B_{2 \lambda}(0)} d z \int_{B_{2 \lambda}\left(\xi_{0}\right)} d \xi \frac{\left|\Delta(z) \int_{0}^{t} u_{\xi} d t^{\prime}\right|^{2}\left|\psi_{3}(b) \widetilde{\vartheta}_{\sigma \xi \xi}\right|^{2}}{|z|^{3+2 \alpha}} \\
& +c \int_{B_{2 \lambda}(0)} d z \int_{B_{2 \lambda}\left(\xi_{0}\right)} d \xi \frac{\left|\int_{0}^{t} u_{\xi} d t^{\prime}\right|^{2}\left|\Delta(z) \psi_{3}(b)\right|^{2}\left|\widetilde{\vartheta}_{\sigma \xi \xi}\right|^{2}}{|z|^{3+2 \alpha}} \\
& +c \int_{B_{2 \lambda}(0)} d z \int_{B_{2 \lambda}\left(\xi_{0}\right)} d \xi \frac{\left|\int_{0}^{t} u_{\xi} d t^{\prime}\right|^{2}\left|\psi_{3}(b) \Delta \widetilde{\vartheta}_{\sigma \xi \xi}\right|^{2}}{|z|^{3+2 \alpha}}=I_{4}^{1}+I_{4}^{2}+I_{4}^{3}
\end{aligned}
$$

where

$$
\begin{aligned}
I_{4}^{1} \leq & \psi_{8}(a)\left[\left(\int_{B_{2 \lambda}(0)} d z \int_{B_{2 \lambda}\left(\xi_{0}\right)} d \xi \frac{\left|\widetilde{\vartheta}_{\sigma \xi \xi}\right|^{2 p}}{|z|^{3-\delta p}}\right)^{1 /(2 p)}\right]^{2} \\
\times & {\left[\left(\int_{\mathbb{R}^{3}} d z \int_{B_{2 \lambda}\left(\xi_{0}\right)} d \xi \frac{\left|\Delta(z) \int_{0}^{t} u_{\xi} d t^{\prime}\right|^{2 p^{\prime}}}{|z|^{3+2 p^{\prime} \alpha+\delta p^{\prime}}}\right)^{1 /\left(2 p^{\prime}\right)}\right]^{2}, }
\end{aligned}
$$

$\delta>0$ is sufficiently small and $1 / p+1 / p^{\prime}=1$. Hence, we get

$$
I_{4}^{1} \leq \psi_{9}(a)\left\|\widetilde{\vartheta}_{\sigma}\right\|_{2+\alpha, \widetilde{\Omega}}^{2} \int_{0}^{t}\|u\|_{2+\alpha, \Omega}^{2} d t^{\prime}
$$

$I_{4}^{2}$ is estimated in the same way, and

$$
\begin{aligned}
I_{4}^{3} & \leq \psi_{10}(a)\left|\int_{0}^{t} u_{\xi} d t^{\prime}\right|_{\infty, \Omega}^{2} \int_{\mathbb{R}^{3}} d z \int_{B_{2 \lambda}\left(\xi_{0}\right)} d \xi \frac{\left|\Delta(z) \widetilde{\vartheta}_{\sigma \xi \xi}\right|^{2}}{|z|^{3+2 \alpha}} \\
& \leq \psi_{11}(a) \int_{0}^{t}\|u\|_{2+\alpha, \Omega}^{2} d t^{\prime}\left\|\widetilde{\vartheta}_{\sigma}\right\|_{2+\alpha, \widetilde{\Omega}}^{2} \cdot
\end{aligned}
$$

Hence

$$
I_{2}^{6} \leq \psi_{12}(a) \int_{0}^{t}\|u\|_{2+\alpha, \Omega}^{2} d t^{\prime}\left\|\widetilde{\vartheta}_{\sigma}\right\|_{2+\alpha, \widetilde{\Omega}}^{2}
$$


Finally,

$$
\begin{aligned}
I_{2}^{3} \leq & \psi_{13}(a)\left(\left\|\vartheta_{\sigma}\right\|_{\alpha, \widetilde{\Omega}}^{2}+\left\|\eta_{\sigma}\right\|_{\alpha, \widetilde{\Omega}}^{2}+1\right) \\
& \cdot\left(\left\|\eta_{\sigma}\right\|_{1+\alpha, \widetilde{\Omega}}^{2}+\left\|\vartheta_{\sigma}\right\|_{1+\alpha, \widetilde{\Omega}}^{2}\right)\|u\|_{2+\alpha, \tilde{\Omega}}^{2} \int_{0}^{t}\|u\|_{2+\alpha, \Omega}^{2} d t^{\prime} .
\end{aligned}
$$

From estimates (3.5)-(3.18) we obtain (3.2).

Our aim is to estimate the sum $\|\widetilde{u}\|_{2+\alpha, \widetilde{\Omega}}^{2}+\left\|\widetilde{\vartheta}_{\sigma}\right\|_{2+\alpha, \widetilde{\Omega}}^{2}+\left\|\widetilde{\eta}_{\sigma}\right\|_{1+\alpha, \widetilde{\Omega}}^{2}$ but we see that on the left-hand side of inequality (3.2) only the seminorms

$$
\int_{\mathbb{R}^{3}} d z \frac{\left\|\Delta^{2}(z) \widetilde{u}\right\|_{1, B_{2 \lambda}\left(\xi_{0}\right)}^{2}}{|z|^{3+2(1+\alpha)}} \text { and } \int_{\mathbb{R}^{3}} d z \int_{B_{2 \lambda}\left(\zeta_{0}\right)} d \xi \frac{p_{2}\left|\nabla\left(\Delta^{2}(z) \widetilde{\vartheta}_{\sigma}\right)\right|^{2}}{|z|^{3+2(1+\alpha)}}
$$

occur. Therefore we have to complete (3.2) by deriving an estimate for $\left\|\widetilde{\eta}_{\sigma}\right\|_{1+\alpha, \widetilde{\Omega}}^{2}+\|\widetilde{u}\|_{1, \widetilde{\Omega}}^{2}+\left\|\nabla \widetilde{\vartheta}_{\sigma}\right\|_{0, \widetilde{\Omega}}^{2}$.

Thus, we obtain

Lemma 3.3. Let the assumptions of Theorem 3.1 be satisfied. Then

$$
\begin{aligned}
& 19) \quad \frac{1}{2} \frac{d}{d t} \int_{\widetilde{\Omega}}\left(\eta \widetilde{u}^{2}+\frac{p_{1}}{\varrho_{e}} \widetilde{\eta}_{\sigma}^{2}+\frac{p_{2} \eta c_{v}(\eta, \vartheta)}{\theta_{e} p_{\vartheta}\left(\varrho_{e}, \theta_{e}\right)} \widetilde{\vartheta}_{\sigma}^{2}\right) d \xi \\
& +\frac{1}{2} \frac{d}{d t} \int_{\mathbb{R}^{3}} d z \int_{B_{2 \lambda}\left(\xi_{0}\right)} d \xi\left(\frac{\left.\eta \Delta^{2}(z) \widetilde{u}\right|^{2}}{|z|^{3+2(1+\alpha)}}+\frac{p_{1}\left|\Delta^{2}(z) \widetilde{\eta}_{\sigma}\right|^{2}}{\varrho_{e}|z|^{3+2(1+\alpha)}}\right. \\
& \left.+\frac{p_{2} \eta c_{v}(\eta, \vartheta)}{\theta_{e} p_{\vartheta}\left(\varrho_{e}, \theta_{e}\right)} \frac{\left|\Delta^{2}(z) \widetilde{\vartheta}_{\sigma}\right|^{2}}{|z|^{3+2(1+\alpha)}}\right) \\
& +c_{2}\left(\|\widetilde{u}\|_{2+\alpha, \widetilde{\Omega}}^{2}+\left\|\widetilde{\vartheta}_{\sigma}\right\|_{2+\alpha, \widetilde{\Omega}}^{2}+\left\|\widetilde{\eta}_{\sigma}\right\|_{1+\alpha, \widetilde{\Omega}}^{2}\right) \\
& \leq \varepsilon\left\|\widetilde{u}_{t}\right\|_{\alpha, \widetilde{\Omega}}^{2}+\varepsilon\left(\|u\|_{2+\alpha, \widetilde{\Omega}}^{2}+\left\|\eta_{\sigma}\right\|_{1+\alpha, \widetilde{\Omega}}^{2}+\left\|\vartheta_{\sigma}\right\|_{1+\alpha, \widetilde{\Omega}}^{2}\right) \\
& +c\|\widetilde{k}\|_{\alpha, \widetilde{\Omega}}^{2}+c(\varepsilon)\left(\|u\|_{0, \widetilde{\Omega}}^{2}+\left\|\widetilde{\eta}_{\sigma}\right\|_{0, \widetilde{\Omega}}^{2}+\left\|\widetilde{\vartheta}_{\sigma}\right\|_{0, \widetilde{\Omega}}^{2}\right) \\
& \quad+c X_{1}\left[X_{2}\left(1+X_{2}\right)+\left(1+X_{2}^{2}\right) \int_{0}^{t}\|u\|_{2+\alpha, \Omega}^{2} d t^{\prime}\right]+c\left\|\eta_{\sigma}\right\|_{1+\alpha, \Omega}^{2}\left\|\widetilde{u}_{t}\right\|_{\alpha, \widetilde{\Omega}}^{2},
\end{aligned}
$$

where $c_{2}>0$ is a constant.

Proof. Multiply equations $(2.6)_{1}$ by $\widetilde{u}_{i}(i=1,2,3)$ and equations $(2.6)_{2}-$ $(2.6)_{3}$ by $\frac{p_{1}}{\varrho_{e}} \widetilde{\eta}_{\sigma}$ and $\frac{p_{2}}{\theta_{e} p_{\vartheta}\left(\varrho_{e}, \theta_{e}\right)} \widetilde{\vartheta}_{\sigma}$, respectively. Then integrating the resulting equations over $\widetilde{\Omega}$, using the boundary condition $\left.\widetilde{u}\right|_{\partial \widetilde{\Omega}}=0$, the equation of continuity, the Korn inequality and relation (1.5), and next adding these 
inequalities we get

$$
\begin{aligned}
& \frac{1}{2} \frac{d}{d t} \int_{\widetilde{\Omega}}\left(\eta \widetilde{u}^{2}+\frac{p_{1}}{\varrho_{e}} \widetilde{\eta}_{\sigma}^{2}+\frac{p_{2} \eta c_{v}(\eta, \vartheta)}{\theta_{e} p_{\vartheta}\left(\varrho_{e}, \theta_{e}\right)} \widetilde{\vartheta}_{\sigma}^{2}\right) d \xi \\
& \quad+c_{3}\left(\|\widetilde{u}\|_{1, \widetilde{\Omega}}^{2}+\left\|\nabla \widetilde{\vartheta}_{\sigma}\right\|_{0, \widetilde{\Omega}}^{2}\right) \\
& \leq \varepsilon\left(\|\widetilde{u}\|_{1, \widetilde{\Omega}}^{2}+\left\|\eta_{\sigma}\right\|_{0, \widetilde{\Omega}}^{2}+\left\|\vartheta_{\sigma}\right\|_{0, \widetilde{\Omega}}^{2}+\left\|\widetilde{\vartheta}_{\sigma}\right\|_{1, \widetilde{\Omega}}^{2}\right) \\
& \quad+\psi_{17}(1 / \varepsilon, a)\left\{\left(\|\widetilde{u}\|_{2, \Omega}^{2}+\left\|\eta_{\sigma}\right\|_{1, \widetilde{\Omega}}^{2}+\left\|\vartheta_{\sigma}\right\|_{2, \widetilde{\Omega}}^{2}\right) \int_{0}^{t}\|u\|_{2, \Omega}^{2} d t^{\prime}\right. \\
& \quad+\left(\left\|\widetilde{\vartheta}_{\sigma}\right\|_{2, \widetilde{\Omega}}^{2}+1\right)\|\widetilde{u}\|_{1, \Omega}^{2} \int_{0}^{t}\|u\|_{2+\alpha, \Omega}^{2} d t^{\prime} \\
& \quad+\left\|\eta_{\sigma}\right\|_{1, \widetilde{\Omega}}^{2}\left[\left\|\vartheta_{\sigma t}\right\|_{1, \widetilde{\Omega}}^{2}+\left\|\widetilde{\vartheta}_{\sigma}\right\|_{2, \widetilde{\Omega}}^{2}+\|u\|_{2, \widetilde{\Omega}}^{2}\left(1+\left\|\widetilde{\eta}_{\sigma}\right\|_{1, \widetilde{\Omega}}^{2}+\left\|\widetilde{\vartheta}_{\sigma}\right\|_{1, \widetilde{\Omega}}^{2}\right)\right] \\
& \quad+\left\|\vartheta_{\sigma}\right\|_{1, \widetilde{\Omega}}^{2}\left(\|u\|_{2, \widetilde{\Omega}}^{2}+\left\|\widetilde{\vartheta}_{\sigma}\right\|_{2, \widetilde{\Omega}}^{2}+\left\|\vartheta_{\sigma t}\right\|_{1, \widetilde{\Omega}}^{2}\right) \\
& \left.\quad+\|u\|_{1, \widetilde{\Omega}}^{2}\|\widetilde{u}\|_{1, \widetilde{\Omega}}^{2}+\|u\|_{1, \widetilde{\Omega}}^{2}+\left\|\vartheta_{\sigma}\right\|_{0, \widetilde{\Omega}}^{2}+\|\widetilde{k}\|_{0, \widetilde{\Omega}}^{2}\right\}
\end{aligned}
$$

where $c_{3}>0$ is a constant.

Now, using the equivalence of norms for fractional spaces (see [4]) and estimates (3.2) and (3.20) we obtain

$$
\begin{aligned}
\frac{1}{2} \frac{d}{d t} \int_{\widetilde{\Omega}} & \left(\eta \widetilde{u}^{2}+\frac{p_{1}}{\varrho_{e}} \widetilde{\eta}_{\sigma}^{2}+\frac{p_{2} \eta c_{v}(\eta, \vartheta)}{\theta_{e} p_{\vartheta}\left(\varrho_{e}, \theta_{e}\right)} \widetilde{\vartheta}_{\sigma}^{2}\right) d \xi \\
& +\frac{1}{2} \frac{d}{d t} \int_{\mathbb{R}^{3}} d z \int_{B_{2 \lambda}\left(\xi_{0}\right)} d \xi\left(\frac{\left.\eta \Delta^{2}(z) \widetilde{u}\right|^{2}}{|z|^{3+2(1+\alpha)}}+\frac{p_{1}\left|\Delta^{2}(z) \widetilde{\eta}_{\sigma}\right|^{2}}{\varrho_{e}|z|^{3+2(1+\alpha)}}\right. \\
& \left.+\frac{p_{2} \eta c_{v}(\eta, \vartheta)}{\theta_{e} p_{\vartheta}\left(\varrho_{e}, \theta_{e}\right)} \frac{\left|\Delta^{2}(z) \widetilde{\vartheta}_{\sigma}\right|^{2}}{|z|^{3+2(1+\alpha)}}\right)+c_{4}\left(\|\widetilde{u}\|_{2+\alpha, \widetilde{\Omega}}^{2}+\left\|\nabla \widetilde{\vartheta}_{\sigma}\right\|_{1+\alpha, \widetilde{\Omega}}^{2}\right) \\
\leq & \left(\|u\|_{2+\alpha, \widetilde{\Omega}}^{2}+\left\|\eta_{\sigma}\right\|_{1+\alpha, \widetilde{\Omega}}^{2}+\left\|\vartheta_{\sigma}\right\|_{2+\alpha, \widetilde{\Omega}}^{2}\right)+c\|\widetilde{k}\|_{\alpha, \widetilde{\Omega}}^{2} \\
& +c\left(\|u\|_{1+\alpha, \widetilde{\Omega}}^{2}+\left\|\widetilde{\eta}_{\sigma}\right\|_{\alpha, \widetilde{\Omega}}^{2}+\left\|\widetilde{\vartheta}_{\sigma}\right\|_{\alpha, \widetilde{\Omega}}^{2}+\left\|\vartheta_{\sigma}\right\|_{1+\alpha, \widetilde{\Omega}}^{2}+\left\|\eta_{\sigma}\right\|_{0, \widetilde{\Omega}}^{2}\right) \\
& +c X_{1}\left[X_{2}\left(1+X_{2}\right)+\left(1+X_{2}^{2}\right) \int_{0}^{t}\|u\|_{2+\alpha, \Omega}^{2} d t^{\prime}\right] \\
& +c\left\|\eta_{\sigma}\right\|_{1+\alpha, \Omega}^{2}\left\|u_{t}\right\|_{\alpha, \widetilde{\Omega}}^{2},
\end{aligned}
$$

where $X_{1}$ and $X_{2}$ are given by (3.3) and (3.4) respectively, and $c_{4}>0$ is a constant. 
To examine the third term on the r.h.s. of (3.21) we apply the interpolation inequality

$$
\begin{aligned}
&\|u\|_{1+\alpha, \widetilde{\Omega}}^{2}+\left\|\widetilde{\eta}_{\sigma}\right\|_{\alpha, \widetilde{\Omega}}^{2}+\left\|\widetilde{\vartheta}_{\sigma}\right\|_{\alpha, \widetilde{\Omega}}^{2}+\left\|\vartheta_{\sigma}\right\|_{1+\alpha, \widetilde{\Omega}}^{2} \\
& \leq \varepsilon\left(\|u\|_{2+\alpha, \widetilde{\Omega}}^{2}+\left\|\widetilde{\eta}_{\sigma}\right\|_{1+\alpha, \widetilde{\Omega}}^{2}+\left\|\vartheta_{\sigma}\right\|_{1+\alpha, \widetilde{\Omega}}^{2}\right) \\
&+c(\varepsilon)\left(\|u\|_{0, \widetilde{\Omega}}^{2}+\left\|\widetilde{\eta}_{\sigma}\right\|_{0, \widetilde{\Omega}}^{2}+\left\|\widetilde{\vartheta}_{\sigma}\right\|_{0, \widetilde{\Omega}}^{2}\right) .
\end{aligned}
$$

From $(2.6)_{1}$ we have

$$
\begin{aligned}
\left\|\widetilde{\eta}_{\sigma}\right\|_{1+\alpha, \widetilde{\Omega}}^{2} \leq & \varepsilon\left(\|u\|_{2+\alpha, \widetilde{\Omega}}^{2}+\left\|\eta_{\sigma}\right\|_{1+\alpha, \widetilde{\Omega}}^{2}+\left\|\vartheta_{\sigma}\right\|_{1+\alpha, \widetilde{\Omega}}^{2}\right) \\
& +c\left(\|\widetilde{u}\|_{2+\alpha, \widetilde{\Omega}}^{2}+\left\|\widetilde{u}_{t}\right\|_{\alpha, \widetilde{\Omega}}^{2}+\|u\|_{0, \widetilde{\Omega}}^{2}+\left\|\eta_{\sigma}\right\|_{0, \widetilde{\Omega}}^{2}+\left\|\vartheta_{\sigma}\right\|_{0, \widetilde{\Omega}}^{2}\right) \\
& +c \int_{0}^{t}\|u\|_{2+\alpha, \Omega}^{2} d t^{\prime}\left(X_{1}+X_{2}^{2}\right)+c X_{2}^{2} .
\end{aligned}
$$

Inequalities (3.21)-(3.23) imply (3.19).

Let us notice that all the terms on the right-hand side of (3.19) different from $\left\|\widetilde{u}_{t}\right\|_{\alpha, \widetilde{\Omega}}$ contain only spatial derivatives of $\widetilde{u}, \widetilde{\eta}_{\sigma}, \widetilde{\vartheta}_{\sigma}, u, \eta_{\sigma}$ and $\vartheta_{\sigma}$. In what follows we will integrate (3.19) with respect to time over $(0, t)$. Therefore, we have to consider $\int_{0}^{t}\left\|\widetilde{u}_{t}\right\|_{\alpha, \widetilde{\Omega}}^{2} d t^{\prime}$. To do this we use the following interpolation inequality (see [8]):

$$
\int_{0}^{t}\left\|\widetilde{u}_{t^{\prime}}\right\|_{\alpha, \widetilde{\Omega}}^{2} d t \leq \varepsilon \int_{0}^{t}\|\widetilde{u}\|_{2+\alpha, \widetilde{\Omega}}^{2} d t+c(\varepsilon) \int_{\widetilde{\Omega}}\left\|\widetilde{u}_{t^{\prime}}\right\|_{\alpha / 2,(0, t)}^{2} d \xi,
$$

where $c(\varepsilon)$ does not depend on $t$.

We see that in order to obtain an inequality of the form (3.1) for an interior subdomain $\widetilde{\Omega}$ we should estimate the second term on the right-hand side of (3.24). To do this we consider the time differences

$$
\Delta_{t}^{k}(h) f(\xi, t)=\sum_{j=0}^{k} c_{k}^{j}(-1)^{k-j} f(\xi, t+j h), \quad k>\alpha .
$$

The estimate for $\int_{\widetilde{\Omega}}\left\|\widetilde{u}_{t^{\prime}}\right\|_{\alpha / 2,(0, t)}^{2} d \xi$ is established in the following lemma.

Lemma 3.4. Let the assumptions of Theorem 3.1 be satisfied. Then

$$
\begin{aligned}
& c_{5} \int_{\widetilde{\Omega}}\left\|u_{t^{\prime}}\right\|_{\alpha / 2,(0, t)}^{2} d \xi \\
& \quad+\left.\int_{0}^{h_{0}} \frac{d h}{h^{1+\alpha}} \int_{\widetilde{\Omega}}\left(\frac{\mu}{4}\left|\mathbb{S}\left(\Delta_{t^{\prime}} \widetilde{u}\right)\right|^{2}+\frac{\nu-\mu}{2}\left|\operatorname{div} \Delta_{t^{\prime}} \widetilde{u}\right|^{2}\right) d \xi\right|_{t^{\prime}=t-h_{0}}
\end{aligned}
$$




$$
\begin{aligned}
\leq & \varepsilon\left(\|u\|_{2+\alpha, \Omega^{t}}^{2}+\left\|\vartheta_{\sigma}\right\|_{2+\alpha, \Omega^{t}}^{2}+\left\|\eta_{\sigma}\right\|_{1+\alpha, \Omega^{t}}^{2}\right)+d\left\|\widetilde{\vartheta}_{\sigma}\right\|_{2+\alpha, \widetilde{\Omega}^{t}}^{2} \\
& +c(\varepsilon, t)\left[\|u\|_{0, \Omega^{t}}^{2}+\left\|\vartheta_{\sigma}\right\|_{0, \Omega^{t}}^{2}+\left\|\eta_{\sigma}\right\|_{0, \Omega^{t}}^{2}+\|k\|_{0, \Omega^{t}}^{2}\right. \\
& +\|u\|_{2+\alpha, \Omega^{t}}^{2} \sup _{0 \leq t^{\prime} \leq t}\|u\|_{1+\alpha, \Omega}^{2} \\
& +\|u\|_{2+\alpha, \widetilde{\Omega}^{t}}^{2}\left(\|u\|_{2+\alpha, \widetilde{\Omega}^{t}}^{2}+\left\|\vartheta_{\sigma}\right\|_{2+\alpha, \widetilde{\Omega}^{t}}^{2}+\left\|\eta_{\sigma}\right\|_{1+\alpha, \Omega^{t}}^{2}\right)\left(1+\|u\|_{1+\alpha, \widetilde{\Omega}^{t}}^{2}\right) \\
& +\sup _{0 \leq t^{\prime} \leq t}\|u\|_{1+\alpha, \widetilde{\Omega}^{2}}^{2}\left(\left\|\vartheta_{\sigma}\right\|_{\alpha, \widetilde{\Omega}^{t}}^{2}+\left\|\eta_{\sigma}\right\|_{1+\alpha, \widetilde{\Omega}^{t}}^{2}\right) \\
& \left.+\left\|\vartheta_{\sigma}\right\|_{2+\alpha, \widetilde{\Omega}^{t}}^{2}\left(\left\|\widetilde{\vartheta}_{\sigma}\right\|_{1+\alpha, \widetilde{\Omega}^{t}}^{2}+\left\|\widetilde{\eta}_{\sigma}\right\|_{1+\alpha, \widetilde{\Omega}^{t}}^{2}\right)\right],
\end{aligned}
$$

where $t \leq T, c_{5}>0$ is a constant, and $c(\varepsilon, t)$ is a positive continuous function increasing with respect to $t$.

Proof. Applying $\Delta_{t}(h)$ to $(2.6)_{1}$ we obtain

$$
\eta \Delta_{t} \widetilde{u}_{i t}-\nabla_{j} T_{i j}\left(\Delta_{t} \widetilde{u}, \Delta_{t} \widetilde{p}_{\sigma}\right)=\eta \Delta_{t} \widetilde{u}_{i t}-\Delta_{t}\left(\eta \widetilde{u}_{i t}\right)+\Delta_{t} k_{1 i} .
$$

In the same way as in [5] from (3.26) we get

$$
\begin{aligned}
& \int_{0}^{h_{0}} \frac{d h}{h^{1+\alpha}} \int_{0}^{t-h_{0}} \psi\left(t^{\prime}\right) d t^{\prime} \int_{\widetilde{\Omega}} \eta\left|\Delta_{t^{\prime}} \widetilde{u}_{t^{\prime}}\right|^{2} d \xi \\
& \quad+\left.\int_{0}^{h_{0}} \frac{d h}{h^{1+\alpha}} \int_{\widetilde{\Omega}}\left(\frac{\mu}{4}\left|\mathbb{S}\left(\Delta_{t^{\prime}} \widetilde{u}\right)\right|^{2}+\frac{\nu-\mu}{2}\left|\operatorname{div} \Delta_{t^{\prime}} \widetilde{u}\right|^{2}\right) d \xi\right|_{t^{\prime}=t-h_{0}} \\
& \quad-\int_{0}^{h_{0}} \frac{d h}{h^{1+\alpha}} \int_{t_{0}}^{2 t_{0}} \psi^{\prime}\left(t^{\prime}\right) d t^{\prime} \int_{\widetilde{\Omega}}\left(\frac{\mu}{4}\left|\mathbb{S}\left(\Delta_{t^{\prime}} \widetilde{u}\right)\right|^{2}+\frac{\nu-\mu}{2}\left|\operatorname{div} \Delta_{t^{\prime}} \widetilde{u}\right|^{2}\right) d \xi \\
& =\int_{0}^{h_{0}} \frac{d h}{h^{1+\alpha}} \int_{0}^{t-h_{0}} \psi\left(t^{\prime}\right) d t^{\prime} \int_{\widetilde{\Omega}}\left[\eta \Delta_{t^{\prime}} \widetilde{u}_{t^{\prime}}-\Delta_{t^{\prime}}\left(\eta \widetilde{u}_{t^{\prime}}\right)\right] \cdot \Delta_{t^{\prime}} \widetilde{u}_{t^{\prime}} d \xi \\
& \quad+\int_{0}^{h_{0}} \frac{d h}{h^{1+\alpha}} \int_{0}^{t-h_{0}} \psi\left(t^{\prime}\right) d t^{\prime} \int_{\widetilde{\Omega}} \Delta_{t^{\prime}} k_{1} \cdot \Delta_{t^{\prime}} \widetilde{u}_{t^{\prime}} d \xi \\
& \quad-\int_{0}^{h_{0}} \frac{d h}{h^{1+\alpha}} \int_{0}^{t-h_{0}} \psi\left(t^{\prime}\right) d t^{\prime} \int_{\widetilde{\Omega}} \nabla\left(\Delta_{t^{\prime}} \widetilde{p}_{\sigma}\right) \cdot \Delta_{t} \widetilde{u}_{t} d \xi \equiv I_{5}+I_{6}+I_{7},
\end{aligned}
$$

where $\mathbb{S}(v)=\left\{v_{i \xi_{j}}+v_{j \xi_{i}}\right\}_{i, j=1,2,3}, 0<h_{0}<t ; \psi\left(t^{\prime}\right)$ is a smooth function vanishing for $t^{\prime} \leq t_{0}$ and equal to 1 for $t^{\prime} \geq 2 t_{0}$. 
First, we estimate $I_{5}$. Using the continuity equation $(1.6)_{2}$ we have

$$
\begin{aligned}
\left|I_{5}\right| & \leq \int_{0}^{h_{0}} \frac{d h}{h^{1+\alpha}} \int_{0}^{t-h_{0}} d t^{\prime} \int_{\widetilde{\Omega}}\left|\Delta_{t^{\prime}}\left(\eta_{\sigma}\right) \widetilde{u}_{t^{\prime}}\right|\left|\Delta_{t^{\prime}} \widetilde{u}_{t^{\prime}}\right| d \xi \\
& \leq \varepsilon\|\widetilde{u}\|_{2+\alpha, \widetilde{\Omega}^{t}}^{2}+c(\varepsilon) \int_{0}^{h_{0}} d h \int_{0}^{t-h_{0}} d t^{\prime} \int_{\widetilde{\Omega}} d \xi \frac{\left|\int_{t^{\prime}}^{t^{\prime}+h} u_{\xi} d \tau\right|^{2}\left|\widetilde{u}_{t^{\prime}}\right|^{2}}{h^{1+\alpha}} \\
& \leq \varepsilon\|\widetilde{u}\|_{2+\alpha, \widetilde{\Omega}^{t}}^{2}+c(\varepsilon) t^{1-\alpha} \int_{0}^{t}\left|u_{\xi}\right|_{\infty, \widetilde{\Omega}}^{2} d t^{\prime} \int_{0}^{t}\left|\widetilde{u}_{t^{\prime}}\right|_{2, \widetilde{\Omega}}^{2} d t^{\prime} \\
& \leq \varepsilon\|\widetilde{u}\|_{2+\alpha, \widetilde{\Omega}^{t}}^{2}+c(\varepsilon) t^{1-\alpha}\|u\|_{2+\alpha, \widetilde{\Omega}^{t}}^{4} .
\end{aligned}
$$

Next, we get

$$
\begin{aligned}
\left|I_{6}\right| \leq & \varepsilon\|\widetilde{u}\|_{2+\alpha, \widetilde{\Omega}^{t}}^{2}+c(\varepsilon) \int_{0}^{h_{0}} \frac{d h}{h^{1+\alpha}} \int_{0}^{t-h_{0}} d t^{\prime} \int_{\widetilde{\Omega}} d \xi\left(\left|\Delta_{t^{\prime}} u\right|^{2}+\left|\Delta_{t^{\prime}} \eta_{\sigma}\right|^{2}\right. \\
& \left.+\left|\Delta_{t^{\prime}} \vartheta_{\sigma}\right|^{2}+\left|\Delta_{t} u_{\xi}\right|^{2}\right) \\
& +\left.\left.c(\varepsilon) \int_{0}^{h_{0}} \frac{d h}{h^{1+\alpha}} \int_{0}^{t-h_{0}} d t^{\prime} \int_{\widetilde{\Omega}} d \xi\right|^{t^{\prime}+h} \int_{t^{\prime}} u_{\xi} d \tau\right|^{2}\left(|u|^{2}+\left|\eta_{\sigma}\right|^{2}+\left|\vartheta_{\sigma}\right|^{2}\right. \\
& \left.+\left|u_{\xi}\right|^{2}+\left|\widetilde{u}_{\xi \xi}\right|^{2}+\left|\widetilde{\eta}_{\sigma \xi}\right|^{2}+\left|\widetilde{\vartheta}_{\sigma \xi}\right|^{2}\right) \\
& +c(\varepsilon) \int_{0}^{h_{0}} \frac{d h}{h^{1+\alpha}} \int_{0}^{t-h_{0}} d t^{\prime} \int_{\widetilde{\Omega}} d \xi\left|\int_{0}^{t^{\prime}} u_{\xi} d \tau\right|^{2} \\
& \cdot\left(\left|\Delta_{t} \widetilde{u}_{\xi \xi}\right|^{2}+\left|\Delta_{t} \widetilde{\eta}_{\sigma \xi}\right|^{2}+\left|\Delta_{t} \widetilde{\vartheta}_{\sigma \xi}\right|^{2}\right) \\
& +\left.\left.c(\varepsilon) \int_{0}^{h_{0}} \frac{d h}{h^{1+\alpha}} \int_{0}^{t-h_{0}} d t^{\prime} \int_{\widetilde{\Omega}} d \xi\right|^{t^{\prime}+h} \int_{t^{\prime}} u_{\xi} d \tau\right|^{2}\left|\int_{0}^{t} u_{\xi \xi} d \tau\right|^{2} \\
& \cdot\left(\left|\widetilde{u}_{\xi}\right|^{2}+\left|\widetilde{\eta}_{\sigma}\right|^{2}+\left|\widetilde{\vartheta}_{\sigma}\right|^{2}\right) \\
& +\left.\left.c(\varepsilon) \int_{0}^{h_{0}} \frac{d h}{h^{1+\alpha}} \int_{0}^{t-h_{0}} d t^{\prime} \int_{\widetilde{\Omega}} d \xi\right|_{t^{\prime}} ^{t^{\prime}+h} u_{\xi \xi} d \tau\right|^{2}\left(\left|\widetilde{u}_{\xi}\right|^{2}+\left|\widetilde{\eta}_{\sigma}\right|^{2}+\left|\widetilde{\vartheta}_{\sigma}\right|^{2}\right) \\
& +c(\varepsilon) \int_{0}^{h_{0}} \frac{d h}{h^{1+\alpha}} \int_{0}^{t-h_{0}} d t^{\prime} \int_{\widetilde{\Omega}} d \xi\left|\int_{0}^{t^{\prime}} u_{\xi \xi} d \tau\right|^{2} \\
& \cdot\left(\left|\Delta_{t} \widetilde{u}_{\xi}\right|^{2}+\left|\Delta_{t} \widetilde{\eta}_{\sigma}\right|^{2}+\left|\Delta_{t} \widetilde{\vartheta}_{\sigma}\right|_{2+\alpha}^{2}\right) \\
& +\widetilde{\Omega}^{t}+\sum_{i=1}^{6} I_{6}^{i} .
\end{aligned}
$$


Let us estimate the terms on the r.h.s. of (3.29). We obtain

$$
\begin{aligned}
I_{6}^{1} & \leq c \int_{0}^{h_{0}} \frac{d h}{h^{1+\alpha}} \int_{0}^{t-h_{0}} d t^{\prime} \int_{\widetilde{\Omega}} d \xi\left(\left|\int_{t^{\prime}}^{t^{\prime}+h} u_{\tau} d \tau\right|^{2}+\left|\int_{t^{\prime}}^{t^{\prime}+h} \vartheta_{\sigma \tau} d \tau\right|^{2}\right) \\
& \leq c t^{1-\alpha}\left(\left\|u_{t^{\prime}}\right\|_{0, \widetilde{\Omega}^{t}}^{2}+\left\|\vartheta_{\sigma t^{\prime}}\right\|_{0, \widetilde{\Omega}^{t}}^{2}\right) .
\end{aligned}
$$

From $(1.6)_{1}$ and $(1.6)_{3}$ we get respectively

$$
\begin{aligned}
\left\|u_{t^{\prime}}\right\|_{0, \Omega}^{2} \leq & \varepsilon_{1}^{1-\varkappa}\left(\|u\|_{2+\alpha, \Omega}^{2}+\left\|\eta_{\sigma}\right\|_{1+\alpha, \Omega}^{2}+\left\|\vartheta_{\sigma}\right\|_{1+\alpha, \Omega}^{2}\right) \\
& +c \varepsilon_{1}^{-\varkappa}\left(\|u\|_{0, \Omega}^{2}+\left\|\eta_{\sigma}\right\|_{0, \Omega}^{2}+\left\|\vartheta_{\sigma}\right\|_{0, \Omega}^{2}\right) \\
& +c\|u\|_{2+\alpha, \Omega^{t}}^{2}\|u\|_{1+\alpha, \Omega}^{2}
\end{aligned}
$$

and

$$
\begin{aligned}
\left\|\vartheta_{\sigma t^{\prime}}\right\|_{0, \Omega}^{2} \leq & \varepsilon_{1}^{1-\varkappa}\left(\|u\|_{1+\alpha, \Omega}^{2}+\left\|\vartheta_{\sigma}\right\|_{2+\alpha, \Omega}^{2}\right) \\
& +c \varepsilon_{1}^{-\varkappa}\left(\|u\|_{0, \Omega}^{2}+\left\|\vartheta_{\sigma}\right\|_{0, \Omega}^{2}\right) \\
& +c\left(\|k\|_{0, \Omega}^{2}+\|u\|_{2+\alpha, \Omega}^{2}\|u\|_{1, \Omega}^{2}\right) .
\end{aligned}
$$

Using (3.31)-(3.32) in (3.30) and assuming $\varepsilon_{1}=\left(\varepsilon /\left(c t^{1-\alpha}\right)\right)^{1 /(1-\varkappa)}$ yields

$$
\begin{aligned}
I_{6}^{1} \leq & \varepsilon\left(\|u\|_{2+\alpha, \Omega^{t}}^{2}+\left\|\vartheta_{\sigma}\right\|_{2+\alpha, \Omega^{t}}^{2}+\left\|\eta_{\sigma}\right\|_{1+\alpha, \Omega^{t}}^{2}\right) \\
& +c(\varepsilon) t^{(1-\alpha) /(1-\varkappa)}\left(\|u\|_{0, \Omega^{t}}^{2}+\left\|\eta_{\sigma}\right\|_{0, \Omega^{t}}^{2}+\left\|\vartheta_{\sigma}\right\|_{0, \Omega^{t}}^{2}+\|k\|_{0, \Omega^{t}}^{2}\right. \\
& \left.+\|u\|_{2+\alpha, \Omega^{t}}^{2} \sup _{0 \leq t^{t} \leq t}\|u\|_{1+\alpha, \Omega}^{2}\right),
\end{aligned}
$$

where $\varepsilon \in(0,1)$ is sufficiently small.

Next, we have

$$
\begin{aligned}
I_{6}^{2} \leq & c t^{1-\alpha} \int_{0}^{t}|u|_{\infty, \widetilde{\Omega}}^{2} d t^{\prime}\left(\int_{0}^{t}|u|_{2, \widetilde{\Omega}}^{2} d t^{\prime}+\int_{0}^{t}\left|\eta_{\sigma}\right|_{2, \widetilde{\Omega}}^{2} d t^{\prime}+\int_{0}^{t}\left|\vartheta_{\sigma}\right|_{2, \widetilde{\Omega}}^{2} d t^{\prime}\right. \\
& \left.+\int_{0}^{t}\left|u_{\xi}\right|_{2, \widetilde{\Omega}}^{2} d t^{\prime}+\int_{0}^{t}\left|\widetilde{u}_{\xi \xi}\right|_{2, \widetilde{\Omega}}^{2} d t^{\prime}+\int_{0}^{t}\left|\widetilde{\eta}_{\sigma \xi}\right|_{2, \widetilde{\Omega}}^{2} d t^{\prime}+\int_{0}^{t}\left|\widetilde{\vartheta}_{\sigma \xi}\right|_{2, \widetilde{\Omega}}^{2} d t^{\prime}\right) \\
\leq & c t^{1-\alpha}\|u\|_{2+\alpha, \widetilde{\Omega}^{t}}^{2}\left(\|u\|_{2, \widetilde{\Omega}^{t}}^{2}+\left\|\eta_{\sigma}\right\|_{1, \widetilde{\Omega}^{t}}^{2}+\left\|\vartheta_{\sigma}\right\|_{1, \widetilde{\Omega}^{t}}^{2} .\right.
\end{aligned}
$$

$I_{6}^{3}$ is estimated in the following way:

$$
\begin{aligned}
I_{6}^{3} \leq & c t \int_{0}^{t}\left|u_{\xi}\right|_{\infty, \widetilde{\Omega}}^{2} d t^{\prime} \int_{0}^{h_{0}} \frac{d h}{h^{1+\alpha}} \\
& \quad \cdot \int_{0}^{t-h_{0}} d t^{\prime}\left(\left|\Delta_{t} \widetilde{u}_{\xi \xi}\right|_{2, \widetilde{\Omega}}^{2}+\left|\Delta_{t} \widetilde{\eta}_{\sigma \xi}\right|_{2, \widetilde{\Omega}}^{2}+\left|\Delta_{t} \widetilde{\vartheta}_{\sigma \xi}\right|_{2, \widetilde{\Omega}}^{2}\right) \\
\leq & c t\|u\|_{2+\alpha, \widetilde{\Omega}^{t}}^{2}\left(\|\widetilde{u}\|_{2+\alpha, \Omega^{t}}^{2}+\left\|\widetilde{\eta}_{\sigma}\right\|_{1+\alpha, \widetilde{\Omega}^{t}}^{2}+\left\|\widetilde{\vartheta}_{\sigma}\right\|_{2+\alpha, \Omega^{t}}^{2}\right) .
\end{aligned}
$$


Next, we get

$$
\begin{aligned}
& I_{6}^{4} \leq c t^{2-\alpha} \int_{0}^{t}\left|u_{\xi}\right|_{\infty, \widetilde{\Omega}}^{2} d t^{\prime} \int_{0}^{t}\left|u_{\xi \xi}\right|_{2, \widetilde{\Omega}}^{2} d t^{\prime} \\
& \cdot\left(\int_{0}^{t}\left|\widetilde{u}_{\xi}\right|_{\infty, \widetilde{\Omega}}^{2} d t^{\prime}+\int_{0}^{t}\left|\widetilde{\eta}_{\sigma}\right|_{\infty, \widetilde{\Omega}}^{2} d t^{\prime}+\int_{0}^{t}\left|\widetilde{\vartheta}_{\sigma}\right|_{\infty, \widetilde{\Omega}}^{2} d t^{\prime}\right) \\
& \leq c t^{2-\alpha}\|u\|_{2+\alpha, \widetilde{\Omega}^{t}}^{4}\left(\|\widetilde{u}\|_{2+\alpha, \widetilde{\Omega}^{t}}^{2}+\left\|\widetilde{\eta}_{\sigma}\right\|_{1+\alpha, \widetilde{\Omega}^{t}}^{2}+\left\|\widetilde{\vartheta}_{\sigma}\right\|_{2+\alpha, \widetilde{\Omega}^{t}}^{2}\right) .
\end{aligned}
$$

Estimating $I_{6}^{5}$ in the same way we obtain

$$
I_{6}^{5} \leq c t^{1-\alpha}\|u\|_{2+\alpha, \widetilde{\Omega}^{t}}^{2}\left(\|\widetilde{u}\|_{2+\alpha, \widetilde{\Omega}^{t}}^{2}+\left\|\widetilde{\eta}_{\sigma}\right\|_{1+\alpha, \widetilde{\Omega}^{t}}^{2}+\left\|\widetilde{\vartheta}_{\sigma}\right\|_{2+\alpha, \widetilde{\Omega}^{t}}^{2}\right) .
$$

Finally, we have

$$
\begin{aligned}
& I_{6}^{6} \leq c t \int_{0}^{t}\left|u_{\xi \xi}\right|_{4, \widetilde{\Omega}}^{2} d t^{\prime} \int_{0}^{h_{0}} \frac{d h}{h^{1+\alpha}} \\
& \quad \cdot \int_{0}^{t-h_{0}} d t^{\prime}\left(\left|\Delta_{t} \widetilde{u}_{\xi}\right|_{4, \widetilde{\Omega}}^{2}+\left|\Delta_{t} \widetilde{\eta}_{\sigma}\right|_{4, \widetilde{\Omega}}^{2}+\left|\Delta_{t} \widetilde{\vartheta}_{\sigma}\right|_{4, \widetilde{\Omega}}^{2}\right) \\
& \leq c t\|u\|_{2+\alpha, \widetilde{\Omega}^{t}}^{2} \int_{0}^{h_{0}} \frac{d h}{h^{1+\alpha}} \\
& \quad \cdot \quad \int_{0}^{t-h_{0}} d t\left[\varepsilon_{1}\left(\left|\Delta_{t} \widetilde{u}_{\xi \xi}\right|_{2, \widetilde{\Omega}^{2}}^{2}+\left|\Delta_{t} \widetilde{\eta}_{\sigma \xi}\right|_{2, \widetilde{\Omega}^{2}}^{2}+\left|\Delta_{t} \widetilde{\vartheta}_{\sigma \xi}\right|_{2, \widetilde{\Omega}^{2}}^{2}\right)\right. \\
& \left.\quad+c\left(\varepsilon_{1}\right)\left(\left|\Delta_{t} \widetilde{u}\right|_{2, \widetilde{\Omega}^{2}}^{2}+\left|\Delta_{t} \widetilde{\eta}_{\sigma}\right|_{2, \widetilde{\Omega}^{2}}^{2}+\left|\Delta_{t} \widetilde{\vartheta}_{\sigma}\right|_{2, \widetilde{\Omega}^{2}}^{2}\right)\right] \\
& \leq c t\|u\|_{2+\alpha, \widetilde{\Omega}^{t}}^{2}\left(\|\widetilde{u}\|_{2+\alpha, \widetilde{\Omega}^{t}}^{2}+\left\|\widetilde{\eta}_{\sigma}\right\|_{1+\alpha, \widetilde{\Omega}^{t}}^{2}+\left\|\widetilde{\vartheta}_{\sigma}\right\|_{2+\alpha, \widetilde{\Omega}^{t}}^{2}\right) .
\end{aligned}
$$

Summarizing the above considerations we get

$$
\begin{aligned}
\left|I_{6}\right| \leq & \varepsilon\left(\|u\|_{2+\alpha, \Omega^{t}}^{2}+\left\|\vartheta_{\sigma}\right\|_{2+\alpha, \Omega^{t}}^{2}+\left\|\eta_{\sigma}\right\|_{1+\alpha, \Omega^{t}}^{2}\right) \\
& +c(\varepsilon, t)\left[\|u\|_{0, \Omega^{t}}^{2}+\left\|\vartheta_{\sigma}\right\|_{0, \Omega^{t}}^{2}+\left\|\eta_{\sigma}\right\|_{0, \Omega^{t}}^{2}+\|k\|_{0, \Omega^{t}}^{2}\right. \\
& +\|u\|_{2+\alpha, \Omega^{t}}^{2} \sup _{0 \leq t^{\prime} \leq t}\|u\|_{1+\alpha, \Omega}^{2}+\|u\|_{2+\alpha, \widetilde{\Omega}^{t}}^{2}\left(\|u\|_{2+\alpha, \widetilde{\Omega}^{t}}^{2}\right. \\
& \left.\left.+\left\|\vartheta_{\sigma}\right\|_{2+\alpha, \widetilde{\Omega}^{t}}^{2}+\left\|\eta_{\sigma}\right\|_{1+\alpha, \Omega^{t}}^{2}\right)\left(1+\|u\|_{1+\alpha, \widetilde{\Omega}^{t}}^{2}\right)\right],
\end{aligned}
$$

where $c(\varepsilon, t)$ is a positive continuous function increasing with respect to $t$.

In the same way we estimate $I_{7}$ and we get

$$
\begin{aligned}
\left|I_{7}\right| \leq & \varepsilon\left(\|u\|_{2+\alpha, \Omega^{t}}^{2}+\left\|\vartheta_{\sigma}\right\|_{2+\alpha, \Omega^{t}}^{2}\right)+d\left\|\widetilde{\vartheta}_{\sigma}\right\|_{2+\alpha, \widetilde{\Omega}^{t}}^{2} \\
& +c(\varepsilon, t)\left[\|u\|_{0, \Omega^{t}}^{2}+\left\|\vartheta_{\sigma}\right\|_{0, \Omega^{t}}^{2}+\|k\|_{0, \Omega^{t}}^{2}\right.
\end{aligned}
$$




$$
\begin{aligned}
& +\sup _{0 \leq t^{\prime} \leq t}\|u\|_{1+\alpha, \widetilde{\Omega}^{2}}^{2}\left(\left\|\vartheta_{\sigma}\right\|_{\alpha, \widetilde{\Omega}^{t}}^{2}+\left\|\eta_{\sigma}\right\|_{1+\alpha, \widetilde{\Omega}^{t}}^{2}\right) \\
& +\sup _{0 \leq t^{\prime} \leq t}\|u\|_{1+\alpha, \Omega^{t}}^{2}\|u\|_{2+\alpha, \widetilde{\Omega}^{t}}^{2} \\
& +\|u\|_{2+\alpha, \widetilde{\Omega}^{t}}^{2}\left(\|u\|_{2+\alpha, \widetilde{\Omega}^{t}}^{2}+\left\|\widetilde{\vartheta}_{\sigma}\right\|_{2, \widetilde{\Omega}^{t}}^{2}+\left\|\widetilde{\eta}_{\sigma}\right\|_{1, \widetilde{\Omega}^{t}}^{2}\right) \\
& \left.+\left\|\vartheta_{\sigma}\right\|_{2+\alpha, \widetilde{\Omega}^{t}}^{2}\left(\left\|\widetilde{\vartheta}_{\sigma}\right\|_{1+\alpha, \widetilde{\Omega}^{t}}^{2}+\left\|\widetilde{\eta}_{\sigma}\right\|_{1+\alpha, \widetilde{\Omega}^{t}}^{2}\right)\right],
\end{aligned}
$$

where $d$ is given in (2.4).

Thus, taking into account (3.27), (3.28), (3.33) and (3.34) we get (3.25).

This completes the proof.

REMARK 3.5. In [5], where the motion of a compressible barotropic viscous fluid in a fixed domain was considered, an estimate analogous to (3.25) was obtained. However, in contrast to the estimate obtained in [5] inequality $(3.25)$ is derived with a function $c(\varepsilon, t)$ on the right-hand side increasing with respect to $t$. This is essential to the proof of the global existence of solutions to problem (1.1) (see [12]) and insignificant for the proof of the global existence for the problem considered in [5].

We also have to estimate $\int_{\widetilde{\Omega}}\left\|\widetilde{\vartheta}_{\sigma t^{\prime}}\right\|_{\alpha / 2,(0, t)}^{2} d \xi$. To do this we apply $\Delta_{t^{\prime}}(h)$ to $(2.6)_{3}$ and then multiply the equation by $\Delta_{t^{\prime}} \widetilde{\vartheta}_{\sigma t^{\prime}}$. The result of the calculations similar to those of Lemma 3.4 is the lemma below.

LEMma 3.6. Let the assumptions of Theorem 3.1 be satisfied. Then

$$
\begin{aligned}
& c_{6} \int_{\widetilde{\Omega}}\left\|\widetilde{\vartheta}_{\sigma t^{\prime}}\right\|_{\alpha / 2,(0, t)}^{2} d \xi+\left.\frac{\varkappa}{2} \int_{0}^{h_{0}} \frac{d h}{h^{1+\alpha}} \int_{\widetilde{\Omega}}\left|\nabla\left(\Delta_{t^{\prime}} \widetilde{\vartheta}_{\sigma}\right)\right|^{2} d \xi\right|_{t^{\prime}=t-h_{0}} \\
& \leq \varepsilon\left(\left\|\vartheta_{\sigma}\right\|_{2+\alpha, \Omega^{t}}^{2}+\|u\|_{2+\alpha, \Omega^{t}}^{2}+\left\|\eta_{\sigma}\right\|_{1+\alpha, \Omega^{t}}^{2}\right) \\
&+d\left\|\widetilde{\vartheta}_{\sigma}\right\|_{2+\alpha, \widetilde{\Omega}^{t}}^{2}+c(\varepsilon, t)\left[\|u\|_{0, \Omega^{t}}^{2}+\left\|\vartheta_{\sigma}\right\|_{0, \Omega^{t}}^{2}\right. \\
&+\left\|\eta_{\sigma}\right\|_{0, \Omega^{t}}^{2}+\|k\|_{\alpha, \Omega^{t}}^{2}+\|\bar{\vartheta}\|_{\alpha+1 / 2, S^{t}}^{2} \\
&+\|u\|_{2+\alpha, \widetilde{\Omega}^{t}}^{2}\left(\|u\|_{2+\alpha, \widetilde{\Omega}^{t}}^{2}+\sup _{0 \leq t^{\prime} \leq t}\left\|\vartheta_{\sigma}\right\|_{1+\alpha, \widetilde{\Omega}^{2}}^{2} \sup _{0 \leq t^{\prime} \leq t}\left\|\eta_{\sigma}\right\|_{1+\alpha, \widetilde{\Omega}}^{2}\right. \\
&+\sup _{0 \leq t^{\prime} \leq t}\|u\|_{1+\alpha, \widetilde{\Omega}}^{2}+\|u\|_{1+\alpha, \widetilde{\Omega}^{t}}^{2} \sup _{0 \leq t^{\prime} \leq t}\|u\|_{1+\alpha, \widetilde{\Omega}}^{2}+\left\|\widetilde{\vartheta}_{\sigma}\right\|_{2+\alpha, \widetilde{\Omega}^{t}}^{2} \\
&+\|u\|_{2+\alpha, \widetilde{\Omega}^{t}}^{2}\left\|\widetilde{\vartheta}_{\sigma}\right\|_{2+\alpha, \Omega^{t}}^{2}+\|u\|_{2+\alpha, \Omega^{t}}^{2}\|u\|_{1+\alpha, \Omega^{t}}^{2} \\
&\left.+\sup _{0 \leq t^{\prime} \leq t}\|\eta\|_{1+\alpha, \widetilde{\Omega}^{2}}^{2}\left\|\widetilde{k}_{\alpha, \widetilde{\Omega}^{t}}^{2}+\right\| \vartheta_{\sigma}\left\|_{2+\alpha, \widetilde{\Omega}^{t}}^{2}\right\| \widetilde{\vartheta}_{\sigma} \|_{2+\alpha, \widetilde{\Omega}^{t}}^{2}\right]
\end{aligned}
$$

where $c_{6}>0$ is a constant and $c(\varepsilon, t)$ is a positive continuous function increasing with respect to $t$. 
Now let us introduce the quantity

$$
\begin{aligned}
\phi_{1}(t, \widetilde{\Omega})= & \int_{\widetilde{\Omega}}\left(\eta \widetilde{u}^{2}+\frac{p_{1}}{\varrho_{e}} \widetilde{\eta}_{\sigma}^{2}+\frac{p_{2} \eta c_{v}(\eta, \vartheta)}{\theta_{e} p_{\vartheta}\left(\varrho_{e}, \theta_{e}\right)} \widetilde{\vartheta}_{\sigma}^{2}\right) d \xi \\
& +\int_{\mathbb{R}^{3}} d z \int_{B_{2 \lambda}\left(\xi_{0}\right)} d \xi\left(\frac{\eta\left|\Delta^{2}(z) \widetilde{u}\right|^{2}}{|z|^{3+2(1+\alpha)}}+\frac{p_{1}\left|\Delta^{2}(z) \widetilde{\eta}_{\sigma}\right|^{2}}{\varrho_{e}|z|^{3+2(1+\alpha)}}\right. \\
& \left.+\frac{p_{2} \eta c_{v}(\eta, \vartheta)}{\theta_{e} p_{\vartheta}\left(\varrho_{e}, \theta_{e}\right)} \frac{\left|\Delta^{2}(z) \widetilde{\vartheta}_{\sigma}\right|^{2}}{|z|^{3+2(1+\alpha)}}\right) .
\end{aligned}
$$

We see that

$$
\begin{aligned}
\bar{c}_{1}\left(\|\widetilde{u}\|_{1+\alpha, \widetilde{\Omega}}^{2}+\left\|\widetilde{\eta}_{\sigma}\right\|_{1+\alpha, \widetilde{\Omega}}^{2}+\left\|\widetilde{\vartheta}_{\sigma}\right\|_{1+\alpha, \widetilde{\Omega}}^{2}\right) \\
\leq \phi_{1}(t, \widetilde{\Omega}) \leq \bar{c}_{2}\left(\|\widetilde{u}\|_{1+\alpha, \widetilde{\Omega}}^{2}+\left\|\widetilde{\eta}_{\sigma}\right\|_{1+\alpha, \widetilde{\Omega}}^{2}+\left\|\widetilde{\vartheta}_{\sigma}\right\|_{1+\alpha, \widetilde{\Omega}}^{2}\right),
\end{aligned}
$$

and $\bar{c}_{1}, \bar{c}_{2}$ depend on the bound from the estimate for the local solution.

Integrating (3.19) with respect to time and using (3.24) (for $\widetilde{u}$ and $\widetilde{\vartheta}_{\sigma}$ ), (3.25) and (3.35) we finally obtain

Lemma 3.7. Let the assumptions of Theorem 3.1 be satisfied. Then, for $t \leq T$

$$
\begin{aligned}
& \phi_{1}(t, \widetilde{\Omega})+c_{7}\left(\|\widetilde{u}\|_{2+\alpha, \widetilde{\Omega}^{t}}^{2}+\left\|\widetilde{\vartheta}_{\sigma}\right\|_{2+\alpha, \widetilde{\Omega}^{t}}^{2}+\int_{0}^{t}\left\|\widetilde{\eta}_{\sigma}\right\|_{1+\alpha, \widetilde{\Omega}}^{2} d t^{\prime}\right) \\
& \leq \varepsilon\left(\|u\|_{2+\alpha, \widetilde{\Omega}^{t}}^{2}+\left\|\vartheta_{\sigma}\right\|_{2+\alpha, \widetilde{\Omega}^{t}}^{2}+\left\|\eta_{\sigma}\right\|_{1+\alpha, \widetilde{\Omega}^{t}}^{2}\right) \\
&+c(\varepsilon, t)\left\{\|u\|_{0, \widetilde{\Omega}^{t}}^{2}+\left\|\vartheta_{\sigma}\right\|_{0, \widetilde{\Omega}^{t}}^{2}+\left\|\eta_{\sigma}\right\|_{0, \widetilde{\Omega}^{t}}^{2}\right. \\
&+\left(1+\sup _{t^{\prime}}\|\eta\|_{1+\alpha, \Omega}^{2}\right)\|k\|_{\alpha, \widetilde{\Omega}^{t}}^{2}+\|\bar{\vartheta}\|_{1 / 2+\alpha, S^{t}}^{2} \\
&\left.+X_{3}\left[X_{4}\left(1+X_{4}\right)+\left(1+X_{4}^{2}\right) X_{3}\right]\right\}+\phi_{1}(0, \widetilde{\Omega}),
\end{aligned}
$$

where

$$
\begin{aligned}
& X_{3}=\|u\|_{2+\alpha, \Omega^{t}}^{2}+\left\|\eta_{\sigma}\right\|_{1+\alpha, \Omega^{t}}^{2}+\left\|\vartheta_{\sigma}\right\|_{2+\alpha, \Omega^{t}}^{2}, \\
& X_{4}=\sup _{t^{\prime} \leq t}\|u\|_{1+\alpha, \Omega}^{2}+\sup _{t^{\prime} \leq t}\left\|\eta_{\sigma}\right\|_{1+\alpha, \Omega}^{2}+\sup _{t^{\prime} \leq t}\left\|\vartheta_{\sigma}\right\|_{1+\alpha, \Omega}^{2},
\end{aligned}
$$

$T$ is the time of local existence, $c(\varepsilon, t)$ is a positive continuous function increasing with respect to $t$, and $c_{7}>0$ is a constant.

Now, we shall derive an estimate analogous to (3.37) which holds in a boundary subdomain $\widehat{\Omega}\left(\xi_{0}\right)$. First, we get an estimate similar to (3.2) for differences of $\widetilde{u}, \widetilde{\vartheta}_{\sigma}$ and $\widetilde{\eta}_{\sigma}$ and their derivatives in directions tangent to $\widehat{S}\left(\xi_{0}\right)$. Then we complete the resulting inequality by estimates which hold for differences in the normal direction to $\widehat{S}\left(\xi_{0}\right)$. 
By $\Delta^{s}(\tau) f(z)$ we denote the $s$ th finite difference of $f$ in the tangent direction such that

$$
\Delta^{s}(\tau) f(z)=\sum_{k=0}^{s}\left(\begin{array}{l}
s \\
k
\end{array}\right)(-1)^{s-k} f\left(z^{\prime}+k \tau, z_{3}\right),
$$

where $z^{\prime}=\left(z_{1}, z_{2}\right), \tau=\left(\tau_{1}, \tau_{2}\right)$.

Similarly $\Delta^{s}(n) f(z)=\sum_{k=0}^{s}\left(\begin{array}{l}s \\ k\end{array}\right)(-1)^{s-k} f\left(z^{\prime}, z_{3}+k n\right)$ is the $s$ th finite difference of $f$ in the normal direction.

Lemma 3.8. Let the assumptions of Theorem 3.1 be satisfied. Then for functions $\widetilde{u}, \widetilde{\eta}_{\sigma}, \widetilde{\vartheta}_{\sigma}$ defined by (2.5) the following estimate holds:

$$
\phi_{2}(t, \widehat{\Omega})+c_{8} \int_{0}^{t} \Phi_{1}\left(t^{\prime}, \widehat{\Omega}\right) d t^{\prime} \leq c \int_{0}^{t} Y d t^{\prime}+\phi_{2}(0, \widehat{\Omega}) \quad \text { for } t \leq T,
$$

where

$$
\begin{aligned}
& =\int_{\mathbb{R}^{2}} d \tau \int_{\widehat{\Omega}\left(\xi_{0}\right)} d z\left(\frac{\widehat{\eta}\left|\Delta^{3}(\tau) \widetilde{u}\right|^{2}}{|\tau|^{2+2(1+\alpha)}}+\frac{p_{1}}{\varrho_{e}} \frac{\left|\Delta^{3}(\tau) \eta_{\sigma}\right|^{2}}{|\tau|^{2+2(1+\alpha)}}+\frac{p_{2} \widehat{\eta} c_{v}(\widehat{\eta}, \widehat{\vartheta})}{\theta_{e} p_{\widehat{\vartheta}}\left(\varrho_{e}, \theta_{e}\right)} \frac{\left|\Delta^{3}(\tau) \widetilde{\vartheta}_{\sigma}\right|^{2}}{|\tau|^{2+2(1+\alpha)}}\right) \\
& +\int_{\widehat{\Omega}\left(\xi_{0}\right)}\left(\widehat{\eta} \widetilde{u}^{2}+\frac{p_{1}}{\varrho_{e}} \widetilde{\eta}_{\sigma}^{2}+\frac{p_{2} \widehat{\eta} c_{v}(\widehat{\eta}, \widehat{\vartheta})}{\theta_{e} p_{\widehat{\vartheta}}\left(\varrho_{e}, \theta_{e}\right)} \widetilde{\vartheta}_{\sigma}^{2}\right) d z \\
& +\frac{2(\mu+\nu)}{\varrho_{e}}\left[\int_{\mathbb{R}^{2}} d \tau \int_{\widehat{\Omega}\left(\xi_{0}\right)} d z \frac{\left|\Delta^{2}(\tau) \widehat{\nabla}_{3} \widetilde{\eta}_{\sigma}\right|^{2}}{|\tau|^{2+2 \alpha}}+\int_{\mathbb{R}_{+}} d n \int_{\widehat{\Omega}\left(\xi_{0}\right)} \frac{\left|\Delta_{3}^{2}(n) \widehat{\nabla}_{3} \widetilde{\eta}_{\sigma}\right|^{2}}{|n|^{1+2 \alpha}}\right] \\
& +\int_{\mathbb{R}^{2}} d \tau \int_{\widehat{\Omega}\left(\xi_{0}\right)} d z\left(\widehat{\eta} \frac{\left|\Delta^{2}(\tau) \widetilde{u}_{z}\right|^{2}}{\left.|\tau|^{2+2 \alpha}+\frac{p_{1}}{\varrho_{e}} \frac{\left|\Delta^{2}(\tau) \widetilde{\eta}_{\sigma z}\right|^{2}}{|\tau|^{2+2 \alpha}}\right)}\right. \\
& +\sigma \int_{\mathbb{R}^{2}} d \tau \int_{\widehat{S}\left(\xi_{0}\right)} d z_{\widehat{S}}\left(4 \sqrt{\widehat{c}} \frac{\Delta^{2}(\tau)\left(\left(H(\cdot, 0)+2 / R_{e}\right) \widehat{\zeta}\right)}{|\tau|^{1+\alpha}}\right. \\
& \left.+\frac{1}{4 \sqrt{\widehat{c}}} \frac{\Delta(\tau)\left(\int_{0}^{t} \Delta^{3}(\tau) \widetilde{u} d t^{\prime} \cdot \widehat{n}\right)}{|\tau|^{1+2+\alpha}}\right)^{2} \\
& +\sigma \int_{\mathbb{R}^{2}} d \tau \int_{\widehat{S}\left(\xi_{0}\right)} d z_{\widehat{S}}\left(\frac{4 \Delta^{2}(\tau)\left(\left(H(\cdot, 0)+2 / R_{e}\right) \widehat{\zeta}\right)}{|\tau|^{1+\alpha}}\right. \\
& \left.+\frac{1}{4} \frac{\sum_{\gamma, \delta=1}^{2} \int_{0}^{t} \Delta^{2}(\tau) \widetilde{u}_{z_{\gamma} z_{\delta}} d t^{\prime} \cdot \widehat{n}}{|\tau|^{1+\alpha}}\right)^{2}
\end{aligned}
$$




$$
\begin{aligned}
& +\frac{\sigma}{4} \int_{\mathbb{R}^{2}} d \tau \int_{\widehat{S}\left(\xi_{0}\right)} d z_{\widehat{S}} \frac{\sum_{\gamma, \delta=1}^{2}\left(\int_{0}^{t} \Delta(\tau) \widetilde{u}_{z_{\gamma} z_{\delta}} d t^{\prime} \cdot \widehat{n}\right)^{2}}{|\tau|^{2+2 \alpha}} \\
& +\frac{\sigma}{4} \int_{\mathbb{R}^{2}} d \tau \int_{\widehat{S}\left(\xi_{0}\right)} d z_{\widehat{S}} \frac{\sum_{\gamma=1}^{2}\left(\int_{0}^{t} \Delta^{3}(\tau) \widetilde{u}_{z_{\gamma}} d t^{\prime} \cdot \widehat{n}\right)^{2}}{|\tau|^{2+2(1+\alpha)}}
\end{aligned}
$$

$\Phi_{1}$ and $Y$ are given by (3.63) and (3.64) respectively, and $c_{8}>0$ is a constant.

Proof. We apply $\Delta^{3}(\tau)$ to $(2.7)_{1}-(2.7)_{3}$ and multiply the resulting equations by $\Delta^{3}(\tau) \widetilde{u}_{i}, \frac{p_{1}}{\varrho_{e}} \Delta^{3}(\tau) \widetilde{\eta}_{\sigma}$ and $\frac{p_{2}}{\theta_{e} p_{\vartheta}\left(\varrho_{e}, \theta_{e}\right)} \Delta^{3}(\tau) \widetilde{\vartheta}_{\sigma}$, respectively. Then integrating each of the equations over $\widehat{\Omega}\left(\xi_{0}\right)$ and with respect to $\tau$ over $\mathbb{R}^{2}$ with the weight $1 /|\tau|^{2+2(1+\alpha)}$ and using (1.5), the Korn inequality and the equation of continuity in $\widehat{\Omega}$, i.e. $\widehat{\eta}_{t}+\widehat{\eta} \widehat{\nabla} \cdot \widehat{u}=0$, we get

$$
\begin{aligned}
& \frac{1}{2} \frac{d}{d t} \int_{\mathbb{R}^{2}} d \tau \int_{\widehat{\Omega}\left(\xi_{0}\right)} d z\left(\frac{\widehat{\eta}\left|\Delta^{3}(\tau) \widetilde{u}\right|^{2}}{|\tau|^{2+2(1+\alpha)}}+\frac{p_{1}}{\varrho_{e}} \frac{\left|\Delta^{3}(\tau) \widetilde{\eta}_{\sigma}\right|^{2}}{|\tau|^{2+2(1+\alpha)}}\right. \\
&\left.+\frac{p_{2} \widehat{\eta} c_{v}}{\theta_{e} p_{\vartheta}\left(\varrho_{e}, \theta_{e}\right)} \frac{\left|\Delta^{3}(\tau) \widetilde{\vartheta}_{\sigma}\right|^{2}}{|\tau|^{2+2(1+\alpha)}}\right)+c_{9} \int_{\mathbb{R}^{2}} d \tau \frac{\left\|\Delta^{3}(\tau) \widetilde{u}\right\|_{1, \widehat{\Omega}\left(\xi_{0}\right)}^{2}}{|\tau|^{2+2(1+\alpha)}} \\
& \quad+\varkappa \int_{\mathbb{R}^{2}} d \tau \int_{\widehat{\Omega}\left(\xi_{0}\right)} d z \frac{p_{2}\left|\Delta^{3}(\tau) \nabla \widetilde{\vartheta}_{\sigma}\right|^{2}}{|\tau|^{2+2(1+\alpha)}} \\
& \quad-\int_{\mathbb{R}^{2}} d \tau \int_{\widehat{S}\left(\xi_{0}\right)} d z_{\widehat{S}} \frac{\mathbb{T}\left(\Delta^{3}(\tau) \widetilde{u}, \Delta^{3}(\tau) \widetilde{p}_{\sigma}\right) \widehat{n}^{\prime} \cdot \Delta^{3}(\tau) \widetilde{u}}{|\tau|^{2+2(1+\alpha)}} \\
& \quad-\int_{\mathbb{R}^{2}} d \tau \int_{\widehat{S}\left(\xi_{0}\right)} d z_{\widehat{S}} \frac{\widehat{n}^{\prime} \cdot \nabla\left(\Delta^{3}(\tau) \widetilde{\vartheta}_{\sigma}\right) \cdot \Delta^{3}(\tau) \widetilde{\vartheta}_{\sigma}}{|\tau|^{2+2(1+\alpha)}} \\
& \leq\left(\|\widehat{u}\|_{2+\alpha, \widehat{\Omega}}^{2}+\left\|\widehat{\eta}_{\sigma}\right\|_{1+\alpha, \widehat{\Omega}}^{2}+\left\|\widehat{\vartheta}_{\sigma}\right\|_{2+\alpha, \widehat{\Omega}}^{2}\right) \\
&+ \psi_{18}(1 / \varepsilon, a)\left[X_{1} X_{2}+X_{1}\left(1+X_{2}^{2}\right) \int_{0}^{t}\|u\|_{2+\alpha, \Omega}^{2} d t^{\prime}\right. \\
&+\left.\left\|\widehat{\eta}_{\sigma}\right\|_{1+\alpha, \Omega}^{2}\left\|\widetilde{u}_{t}\right\|_{\alpha, \widehat{\Omega}}^{2}\right] \\
&+ \psi_{19}(1 / \varepsilon, a)\left(\|\widetilde{u}\|_{1+\alpha, \widehat{\Omega}}^{2}+\left\|\widetilde{\eta}_{\sigma}\right\|_{\alpha, \widehat{\Omega}}^{2}\right. \\
&+\left.\left\|\widetilde{\vartheta}_{\sigma}\right\|_{\alpha, \widehat{\Omega}}^{2}+\left\|\vartheta_{\sigma}\right\|_{1+\alpha, \widehat{\Omega}}^{2}\right)+\psi_{20}(1 / \varepsilon, a)\|\widetilde{k}\|_{\alpha, \widehat{\Omega}}^{2}
\end{aligned}
$$

where $X_{1}$ and $X_{2}$ are given by (3.3) and (3.4) respectively. 
Using the boundary condition $(2.7)_{4}$, definition (2.4) and imbedding (2.11) we obtain

$$
\begin{aligned}
& \int_{\mathbb{R}^{2}} d \tau \int_{\widehat{S}\left(\xi_{0}\right)} d z_{\widehat{S}} \frac{\mathbb{T}\left(\Delta^{3}(\tau) \widetilde{u}, \Delta^{3}(\tau) \widetilde{p}_{\sigma}\right) \widehat{n}^{\prime} \cdot \Delta^{3}(\tau) \widetilde{u}}{|\tau|^{2+2(1+\alpha)}} \\
= & -\frac{\sigma}{2} \frac{d}{d t} \int_{\mathbb{R}^{2}} d \tau \int_{\widehat{S}\left(\xi_{0}\right)} d z_{\widehat{S}} \frac{g_{\widehat{u}}^{\gamma \delta} \int_{0}^{t} \Delta^{3}(\tau) \frac{\partial \widetilde{u}}{\partial z_{\gamma}} d t^{\prime} \cdot \widehat{n} \int_{0}^{t} \Delta^{3}(\tau) \frac{\partial \widetilde{u}}{\partial z_{\delta}} d t^{\prime} \cdot \widehat{n}}{|\tau|^{2+2(1+\alpha)}} \\
& +\sigma \int_{\mathbb{R}^{2}} d \tau \int_{\widehat{S}\left(\xi_{0}\right)} d z_{\widehat{S}} \frac{\Delta^{3}(\tau)\left(\left(\Delta_{\widehat{S}}(0) \widehat{\xi} \cdot \widehat{n}+\left(2 / R_{e}\right) \widehat{n}\right) \widehat{\zeta}\right) \widehat{n} \cdot \Delta^{3}(\tau) \widetilde{u}}{|\tau|^{2+2(1+\alpha)}}+I_{8} \\
\equiv & J_{1}+J_{2}+I_{8},
\end{aligned}
$$

where

$$
\begin{aligned}
& \left|I_{8}\right| \leq \psi_{21}\left(a,\|F\|_{5 / 2+\alpha, \widehat{S}}\right)\left[(\varepsilon+d)\|\widetilde{u}\|_{2+\alpha, \widehat{\Omega}}^{2}\right. \\
& +\varepsilon\left(\left\|\widetilde{\eta}_{\sigma}\right\|_{1+\alpha, \widehat{\Omega}}^{2}+\left\|\widetilde{\vartheta}_{\sigma}\right\|_{1+\alpha, \widehat{\Omega}}^{2}\right) \\
& \quad+\varepsilon_{1}\left\|\int_{0}^{t} \widehat{u} d t^{\prime}\right\|_{5 / 2+\alpha, \widehat{S}}^{2}+\varepsilon_{1}\left\|H(\cdot, 0)+2 / R_{e}\right\|_{1 / 2+\alpha, S}^{2} \\
& \left.+\|\widehat{u}\|_{2+\alpha, \widehat{\Omega}}^{2} \int_{0}^{t}\|\widehat{u}\|_{2+\alpha, \widehat{\Omega}}^{2} d t^{\prime}+\int_{0}^{t}\|\widetilde{u}\|_{2+\alpha, \widehat{\Omega}}^{2} d t^{\prime}\left\|\int_{0}^{t} \widehat{u} d t^{\prime}\right\|_{2+\alpha, \widehat{\Omega}}^{2}+\|\widetilde{u}\|_{0, \widehat{\Omega}}^{2}\right],
\end{aligned}
$$

$\varepsilon_{1}$ and $\varepsilon$ are sufficiently small positive constants, and $d$ is given in (2.4).

Now, we estimate the second term on the right-hand side of (3.43):

$$
\begin{aligned}
J_{2} & =\sigma \int_{\mathbb{R}^{2}} d \tau \int_{\widehat{S}\left(\xi_{0}\right)} d z_{\widehat{S}} \frac{\Delta^{3}(\tau)\left(\left(H(\cdot, 0)+2 / R_{e}\right) \widehat{\zeta}\right) \cdot \Delta^{3}(\tau) \widetilde{u}}{|\tau|^{2+2(1+\alpha)}} \\
& +\frac{2 \sigma}{R_{e}} \int_{\mathbb{R}^{2}} d \tau \int_{\widehat{S}\left(\xi_{0}\right)} d z_{\widehat{S}} \frac{\Delta^{3}(\tau) \Delta_{\widehat{S}}(0) \widehat{\zeta} \cdot\left(\widehat{n}-\widehat{n}_{0}\right) \cdot \Delta^{3}(\tau) \widetilde{u}}{|\tau|^{2+2(1+\alpha)}} \equiv J_{2}^{1}+J_{2}^{2},
\end{aligned}
$$

where $\widehat{n}_{0}\left(z^{\prime}\right)=\widehat{n}\left(z_{1}^{\prime} 0\right)$ and

$$
\left|J_{2}^{2}\right| \leq \varepsilon_{1}\left\|\int_{0}^{t} \widehat{u} d t^{\prime}\right\|_{5 / 2+\alpha, \widehat{S}}^{2}+c\left(\varepsilon_{1}\right)\left(\varepsilon\|\widetilde{u}\|_{2+\alpha, \widehat{\Omega}}^{2}+c(\varepsilon)\|\widetilde{u}\|_{0, \widehat{\Omega}}^{2}\right) .
$$

Next, we obtain

$$
J_{2}^{1}=-\frac{\sigma}{2} \frac{d}{d t} \int_{\mathbb{R}^{2}} d \tau \int_{\widehat{S}\left(\xi_{0}\right)} \frac{2 \Delta^{2}(\tau)\left(\left(H(\cdot, 0)+2 / R_{e}\right) \widehat{\zeta}\right) \cdot \Delta(\tau)\left(\int_{0}^{t} \Delta^{3}(\tau) \widetilde{u} d t^{\prime} \cdot \widehat{n}\right)}{|\tau|^{2+2+(1+\alpha)}}
$$




$$
\begin{aligned}
= & -\frac{\sigma}{2} \frac{d}{d t} \int_{\mathbb{R}^{2}} d \tau \int_{\widehat{S}\left(\xi_{0}\right)} d z_{\widehat{S}}\left(4 \sqrt{\widehat{c}} \frac{\Delta^{2}(\tau)\left(\left(H(\cdot, 0)+2 / R_{e}\right) \widehat{\zeta}\right)}{|\tau|^{1+\alpha}}\right. \\
& \left.+\frac{1}{4 \sqrt{\widehat{c}}} \frac{\Delta(\tau)\left(\int_{0}^{t} \Delta^{3}(\tau) \widetilde{u} d t^{\prime} \cdot \widehat{n}\right)}{|\tau|^{1+2+\alpha}}\right)^{2} \\
& +\frac{\sigma}{32 \bar{c}} \frac{d}{d t} \int_{\mathbb{R}^{2}} d \tau \int_{\widehat{S}\left(\xi_{0}\right)} d z_{\widehat{S}} \frac{\left(\Delta(\tau)\left(\int_{0}^{t} \Delta^{3}(\tau) \widetilde{u} d t^{\prime} \cdot \widehat{n}\right)\right)^{2}}{|\tau|^{2+2(2+\alpha)}}
\end{aligned}
$$

where $\widehat{c}>0$ is a constant such that (see [3], [4])

$$
\begin{aligned}
\int_{\mathbb{R}^{2}} d \tau \int_{\widehat{S}(\xi)} d z_{\widehat{S}} \frac{\left(\Delta(\tau)\left(\int_{0}^{t} \Delta^{3}(\tau) \widetilde{u} d t^{\prime} \cdot \widehat{n}\right)\right)^{2}}{|\tau|^{2+2(2+\alpha)}} \\
\quad \leq \widehat{c} \int_{\mathbb{R}^{2}} d \tau \int_{\widehat{S}\left(\xi_{0}\right)} d z_{\widehat{S}} \frac{\sum_{\gamma=1}^{2}\left(\left(\int_{0}^{t} \Delta^{3}(\tau) \widetilde{u} d t^{\prime} \cdot \widehat{n}\right)_{z_{\gamma}}\right)^{2}}{|\tau|^{2+2(1+\alpha)}}
\end{aligned}
$$

By (3.43), (3.45), (3.47) we get

$$
\begin{aligned}
-\int_{\mathbb{R}^{2}} d \tau \int_{\widehat{S}\left(\xi_{0}\right)} d z_{\widehat{S}} \frac{\mathbb{T}\left(\Delta^{3}(\tau) \widetilde{u}, \Delta^{3}(\tau) \widetilde{p}_{\sigma}\right) \widehat{n}^{\prime} \cdot \Delta^{3}(\tau) \widetilde{u}}{|\tau|^{2+2(1+\alpha)}} \\
=\frac{\sigma}{2} \frac{d}{d t} \int_{\mathbb{R}^{2}} d \tau \int_{\widehat{S}\left(\xi_{0}\right)} d z_{\widehat{S}} \frac{g_{\widehat{u}}^{\gamma \delta} \int_{0}^{t} \Delta^{3}(\tau) \widetilde{u}_{z_{\gamma}} d t^{\prime} \cdot \widehat{n} \int_{0}^{t} \Delta^{3}(\tau) \widetilde{u}_{z_{\delta}} d t^{\prime} \cdot \widehat{n}}{|\tau|^{2+2(1+\alpha)}} \\
+\frac{\sigma}{2} \frac{d}{d t} \int_{\mathbb{R}^{2}} d \tau \int_{\widehat{S}\left(\xi_{0}\right)} d z_{\widehat{S}}\left(4 \sqrt{\widehat{c}} \frac{\Delta^{2}(\tau)\left(\left(H(\cdot, 0)+2 / R_{e}\right) \widehat{\zeta}\right)}{|\tau|^{1+\alpha}}\right. \\
\left.+\frac{1}{4 \sqrt{\widehat{c}}} \frac{\Delta(\tau)\left(\int_{0}^{t} \Delta^{3}(\tau) \widetilde{u} d t^{\prime} \cdot \widehat{n}\right)}{|\tau|^{1+2+\alpha}}\right)^{2} \\
\quad-\frac{\sigma}{32 \widehat{c}} \frac{d}{d t} \int_{\mathbb{R}^{2}} d \tau \int_{\widehat{S}\left(\xi_{0}\right)} d z_{\widehat{S}} \frac{\left(\Delta(\tau)\left(\int_{0}^{t} \Delta^{3}(\tau) \widetilde{u} d t^{\prime} \cdot \widehat{n}\right)^{2}\right.}{|\tau|^{2+2(2+\alpha)}+I_{8}+J_{2}^{2}}
\end{aligned}
$$

where estimates for $I_{8}$ and $J_{2}^{2}$ are given by (3.44) and (3.46), respectively.

Next, using the boundary condition $(2.7)_{5}$ we have

$$
\begin{aligned}
\int_{\mathbb{R}^{2}} d \tau \int_{\widehat{S}\left(\xi_{0}\right)} d z_{\widehat{S}} & \frac{\widehat{n}^{\prime} \cdot \nabla\left(\Delta^{3}(\tau) \widetilde{\vartheta}_{\sigma}\right) \cdot \Delta^{3}(\tau) \widetilde{\vartheta}_{\sigma}}{|\tau|^{2+2(1+\alpha)}} \\
\leq & \psi_{22}\left(a,\|F\|_{2+\alpha, \widehat{S}}\right)\left[(\varepsilon+d)\left\|\widehat{\vartheta}_{\sigma}\right\|_{2+\alpha, \widehat{\Omega}}^{2}+\|\widetilde{\bar{\vartheta}}\|_{\alpha+1 / 2, \widehat{S}}\right. \\
& \left.+\left\|\widehat{\vartheta}_{\sigma}\right\|_{0, \widehat{\Omega}}^{2}+\left\|\widehat{\vartheta}_{\sigma}\right\|_{2+\alpha, \widehat{\Omega}}^{2}\left\|\int_{0}^{t} \widehat{u} d t^{\prime}\right\|_{2+\alpha, \widehat{\Omega}}^{2}\right]
\end{aligned}
$$


Taking into account (3.42), (3.44), (3.46), (3.49), (3.50) and the considerations for interior subdomains we get

$$
\frac{d}{d t} \phi_{3}(t, \widehat{\Omega})+c_{10} \Phi_{2}(t, \widehat{\Omega}) \leq c X,
$$

where

$$
\phi_{3}(t, \widehat{\Omega})=\frac{1}{2} \int_{\mathbb{R}^{2}} d \tau \int_{\widehat{\Omega}\left(\xi_{0}\right)} d z\left(\frac{\widehat{\eta}\left|\Delta^{3}(\tau) \widetilde{u}\right|^{2}}{|\tau|^{2+2(1+\alpha)}}+\frac{p_{1}}{\varrho_{e}} \frac{\left|\Delta^{3}(\tau) \widetilde{\eta}_{\sigma}\right|^{2}}{|\tau|^{2+2(1+\alpha)}}\right.
$$$$
\left.+\frac{p_{2} \widehat{\eta} c_{v}(\widehat{\eta}, \widehat{\vartheta})}{\theta_{e} p_{\widehat{\vartheta}}\left(\varrho_{e}, \theta_{e}\right)} \frac{\left|\Delta^{3}(\tau) \widetilde{\vartheta}_{\sigma}\right|^{2}}{|\tau|^{2+2(1+\alpha)}}\right)+\frac{1}{2} \int_{\widehat{\Omega}\left(\xi_{0}\right)}\left(\widehat{\eta} \widetilde{u}^{2}+\frac{p_{1}}{\varrho_{e}} \widetilde{\eta}_{\sigma}^{2}+\frac{p_{2} \widehat{\eta} c_{v}(\widehat{\eta}, \widehat{\vartheta})}{\theta_{e} p_{\widehat{\vartheta}}\left(\varrho_{e}, \theta_{e}\right)} \widetilde{\vartheta}_{\sigma}^{2}\right) d z
$$$$
+\frac{\sigma}{2} \int_{\mathbb{R}^{2}} d \tau \int_{\widehat{S}\left(\xi_{0}\right)} d z_{\widehat{S}} \frac{g_{\widehat{u}}^{\gamma \delta} \int_{0}^{t} \Delta^{3}(\tau) \frac{\partial \widetilde{u}}{\partial z_{\gamma}} d t^{\prime} \cdot \widehat{n} \int_{0}^{t} \Delta^{3}(\tau) \frac{\partial \widetilde{u}}{\partial z_{\delta}} d t^{\prime} \cdot \widehat{n}}{|\tau|^{2+2(1+\alpha)}}
$$$$
+\frac{\sigma}{2} \int_{\mathbb{R}^{2}} d \tau \int_{\widehat{S}\left(\xi_{0}\right)} d z_{\widehat{S}}\left(4 \sqrt{\widehat{c}} \frac{\Delta^{2}(\tau)\left(\left(H(\cdot, 0)+2 / R_{e}\right) \widehat{\zeta}\right)}{|\tau|^{1+\alpha}}\right.
$$$$
\left.+\frac{1}{4 \sqrt{\widehat{c}}} \frac{\Delta(\tau)\left(\int_{0}^{t} \Delta^{3}(\tau) \widetilde{u} d t^{\prime} \cdot \widehat{n}\right)}{|\tau|^{1+2+\alpha}}\right)^{2}
$$$$
-\frac{\sigma}{32 \bar{c}} \int_{\mathbb{R}^{2}} d \tau \int_{\widehat{S}\left(\xi_{0}\right)} d z_{\widehat{S}} \frac{\left(\Delta(\tau)\left(\int_{0}^{t} \Delta^{3}\left(\tau^{\prime}\right) \widetilde{u} d t^{\prime} \cdot \widehat{n}\right)\right)^{2}}{|\tau|^{2+2(2+\alpha)}},
$$

$$
\begin{aligned}
\Phi_{2}(t, \widehat{\Omega})= & c\left(\|\widetilde{u}\|_{1, \widehat{\Omega}}^{2}+\left\|\widetilde{\vartheta}_{\sigma x}\right\|_{0, \widehat{\Omega}}^{2}+\int_{\mathbb{R}^{2}} d \tau \frac{\left\|\Delta^{3}(\tau) \widetilde{u}\right\|_{1, \widehat{\Omega}\left(\xi_{0}\right)}^{2}}{|\tau|^{2+2(1+\alpha)}}\right. \\
& \left.+\int_{\mathbb{R}^{2}} d \tau \int_{\widehat{\Omega}\left(\xi_{0}\right)} d z \frac{p_{2}\left|\nabla\left(\Delta^{3}(\tau) \widetilde{\vartheta}_{\sigma}\right)\right|^{2}}{|\tau|^{2+2(1+\alpha)}}\right)
\end{aligned}
$$

$$
\begin{aligned}
X= & \psi_{23}\left(a,\|F\|_{5 / 2+\alpha, \widehat{S}}\right)\left\{d\left(\|\widetilde{u}\|_{2+\alpha, \widehat{\Omega}}^{2}+\left\|\widetilde{\vartheta}_{\sigma}\right\|_{2+\alpha, \widehat{\Omega}}^{2}\right)\right. \\
& +\varepsilon\left(\|\widehat{u}\|_{2+\alpha, \widehat{\Omega}}^{2}+\left\|\widehat{\vartheta}_{\sigma}\right\|_{2+\alpha, \widehat{\Omega}}^{2}\right)+\varepsilon\left\|\widetilde{\eta}_{\sigma}\right\|_{1+\alpha, \widehat{\Omega}}^{2}+\varepsilon_{1}\left\|\int_{0}^{t} \widehat{u} d t^{\prime}\right\|_{5 / 2+\alpha, \widehat{S}}^{2} \\
& +A+\left\|\widehat{\vartheta}_{\sigma}\right\|_{2+\alpha, \widehat{\Omega}}^{2}\left\|\int_{0}^{t} \widehat{u} d t^{\prime}\right\|_{2+\alpha, \widehat{\Omega}}^{2}+\varepsilon_{1}\left\|H(\cdot, 0)+2 / R_{e}\right\|_{1 / 2+\alpha, S}^{2} \\
& \left.+X_{1} X_{2}+X_{1}\left(1+X_{2}^{2}\right) \int_{0}^{t}\|\widehat{u}\|_{2+\alpha, \Omega}^{2} d t^{\prime}+\left\|\eta_{\sigma}\right\|_{1+\alpha, \Omega}^{2}\left\|\widetilde{u}_{t}\right\|_{\alpha, \widetilde{\Omega}}^{2}\right\},
\end{aligned}
$$




$$
A=\|\widehat{u}\|_{0, \hat{\Omega}}^{2}+\left\|\widehat{\eta}_{\sigma}\right\|_{0, \hat{\Omega}}^{2}+\left\|\widehat{\vartheta}_{\sigma}\right\|_{0, \hat{\Omega}}^{2}+\|\widehat{k}\|_{\alpha, \hat{\Omega}}^{2}+\|\widetilde{\bar{\vartheta}}\|_{\alpha+1 / 2, \widehat{S}}^{2}
$$

and $X_{1}$ and $X_{2}$ are given by (3.3) and (3.4), respectively.

Now, repeating the argument from Lemma 4.4 of [15] we obtain the estimate

$$
\begin{aligned}
& \quad \frac{1}{2} \frac{d}{d t} \int_{\mathbb{R}^{2}} d \tau \int_{\widehat{\Omega}\left(\xi_{0}\right)} d z\left(\widehat{\eta} \frac{\left|\Delta^{2}(\tau) \widetilde{u}_{z}\right|^{2}}{|\tau|^{2+2 \alpha}}+\frac{p_{1}}{\varrho_{e}} \frac{\left|\Delta^{2}(\tau) \widetilde{\eta}_{\sigma z}\right|^{2}}{|\tau|^{2+2 \alpha}}\right) \\
& +\frac{\sigma}{2} \frac{d}{d t} \int_{\mathbb{R}^{2}} d \tau \int_{\widehat{S}\left(\xi_{0}\right)} d z_{\widehat{S}} \frac{g_{\widehat{u}}^{\gamma \delta} \int_{0}^{t} \Delta^{2}(\tau) \widetilde{u}_{z_{\beta} z_{\gamma}} d t^{\prime} \cdot \widehat{n} \int_{0}^{t} \Delta^{2}(\tau) \widetilde{u}_{z_{\beta} z_{\delta}} d t^{\prime} \cdot \widehat{n}}{|\tau|^{2+2 \alpha}} \\
& +\frac{\sigma}{2} \frac{d}{d t} \int_{\mathbb{R}^{2}} d \tau \int_{\widehat{S}\left(\xi_{0}\right)} d z_{\widehat{S}}\left(\frac{4 \Delta^{2}(\tau)\left(H(\cdot, 0)+2 / R_{e}\right) \widehat{\zeta}}{|\tau|^{1+\alpha}}\right. \\
& \left.+\frac{1}{4} \frac{\sum_{\gamma, \delta=1}^{2} \int_{0}^{t} \Delta^{2}(\tau) \widetilde{u}_{z_{\gamma} z_{\delta}} d t^{\prime} \cdot \widehat{n}}{|\tau|^{1+\alpha}}\right)^{2} \\
& -\frac{\sigma}{32} \frac{d}{d t} \int_{\mathbb{R}^{2}} d \tau \int_{\widehat{S}\left(\xi_{0}\right)} d z_{\widehat{S}} \frac{\sum_{\gamma, \delta=1}^{2}\left(\int_{0}^{t} \Delta^{2}(\tau) \widetilde{u}_{z_{\gamma} z_{\delta}} d t^{\prime}\right)^{2}}{|\tau|^{2+2 \alpha}} \\
& \quad+\frac{\mu}{2} \int_{\mathbb{R}^{2}} \frac{\left\|\Delta^{2}(\tau) \widetilde{u}\right\|_{2, \widehat{\Omega}}^{2}}{|\tau|^{2+2 \alpha}} d \tau+\int_{\mathbb{R}^{2}} \frac{\left\|\Delta^{2}(\tau) \widetilde{\eta}_{\sigma z}\right\|_{0, \widehat{\Omega}}^{2}}{|\tau|^{2+2 \alpha}} d \tau \\
& \leq \varepsilon\left(\left\|\int_{0}^{t} \widetilde{u} d t^{\prime}\right\|_{5 / 2}^{2}\right. \\
& \left.\quad+\left\|H(\cdot, 0)+2 / R_{e}\right\|_{\alpha+1 / 2, S}^{2}+\|R(\cdot, t)-R(\cdot, 0)\|_{5 / 2+\alpha, S^{1}}^{2}\right) \\
& \quad+c\left(\|\widehat{u}\|_{2+\alpha, \widehat{\Omega}}^{2}\left\|_{0, \widehat{\Omega}}^{t}+\right\| \widehat{\eta}_{\sigma}\left\|_{0, \widehat{\Omega}}^{2}+\right\| \widehat{\vartheta}_{\sigma} \|_{0, \widehat{\Omega}}^{2}+c X_{2}\left(X_{1}+\int_{0}^{t}\|u\|_{2+\alpha, \Omega}^{2} d t^{\prime}\right) .\right.
\end{aligned}
$$

Next,

$$
\left\|\int_{0}^{t} \widetilde{u} d t^{\prime}\right\|_{5 / 2+\alpha, \widehat{S}}^{2} \leq c\|R(\cdot, t)-R(\cdot, 0)\|_{5 / 2+\alpha, S^{1}} .
$$

Now, we consider the problem

$$
\begin{array}{ll}
\varkappa \Delta \widetilde{\vartheta}_{\sigma}=\widehat{\eta} c_{v}(\widehat{\eta}, \widehat{\vartheta}) \widetilde{\vartheta}_{\sigma t}+\theta_{e} p_{\widehat{\vartheta}}\left(\varrho_{e}, \theta_{e}\right) \nabla \cdot \widetilde{u}-\widehat{\eta} \widetilde{k}-k_{6} \equiv K_{3} & \text { in } \widehat{\Omega} \\
\widehat{n} \cdot \nabla \widetilde{\vartheta}_{\sigma}=\widetilde{\bar{\vartheta}}+k_{8} \equiv K_{4} & \text { on } \widehat{S}
\end{array}
$$


From Agmon-Douglis-Nirenberg theory (see [2]) we get

$$
\begin{aligned}
\int_{\mathbb{R}^{2}} \frac{\left\|\Delta(\tau) \widetilde{\vartheta}_{\sigma}\right\|_{2, \widehat{\Omega}}^{2}}{|\tau|^{2+2 \alpha}} d \tau & \leq c \int_{\mathbb{R}^{2}} \frac{\|\Delta(\tau) \nabla \cdot \widetilde{u}\|_{1, \widehat{\Omega}}}{|\tau|^{2+2 \alpha}} d \tau \\
& +c \int_{\mathbb{R}^{2}} \frac{\left\|\Delta(\tau) K_{3}\right\|_{0, \widehat{\Omega}}^{2}}{|\tau|^{2+2 \alpha}} d \tau+c \int_{\mathbb{R}^{2}} \frac{\left\|\Delta(\tau) K_{4}\right\|_{1 / 2, \widehat{S}}^{2}}{|\tau|^{2+2 \alpha}} d \tau \\
& \leq c\left(X+\left\|\widetilde{\vartheta}_{\sigma t}\right\|_{\alpha, \widehat{\Omega}}^{2}\right) \equiv c \bar{X}
\end{aligned}
$$

Next, applying $\Delta_{3}(n)$ to $(3.58)_{1}$ we obtain the estimate

$$
\int_{\mathbb{R}_{+}} d n \int_{\widehat{\Omega}\left(\xi_{0}\right)} d z \frac{\left|\Delta_{3}(n) \widetilde{\vartheta}_{\sigma z_{3} z_{3}}\right|^{2}}{|n|^{1+2 \alpha}} \leq c \int_{\mathbb{R}_{+}} d n \int_{\widehat{\Omega}\left(\xi_{0}\right)} d z \frac{\left|\Delta_{3}(n) \widetilde{\vartheta}_{\sigma z z^{\prime}}\right|^{2}}{|n|^{1+2 \alpha}}+C \bar{X}
$$

where the first term on the right-hand side of (3.60) is estimated using the following interpolation inequality:

$$
\begin{aligned}
\int_{\mathbb{R}_{+}} d n \int_{\widehat{\Omega}\left(\xi_{0}\right)} d z \frac{\left|\Delta_{3}(n) \widetilde{\vartheta}_{\sigma z z^{\prime}}\right|^{2}}{|n|^{1+2 \alpha}} \leq & c \int_{\mathbb{R}^{2}} d \tau \int_{\widehat{\Omega}\left(\xi_{0}\right)} \frac{\left|\Delta(\tau) \widetilde{\vartheta}_{\sigma z z^{\prime}}\right|^{2}}{|\tau|^{2+2 \alpha}} d z \\
& +c \int_{\mathbb{R}^{2}} d \tau \int_{\widehat{\Omega}\left(\xi_{0}\right)} \frac{\left|\Delta(\tau) \widetilde{\vartheta}_{\sigma z z}\right|^{2}}{|\tau|^{2+2 \alpha}} d z
\end{aligned}
$$

Repeating the considerations from [5] (see the proof of Lemma 3.2) and taking into account (3.51), (3.56), (3.57), (3.59)-(3.61) we get

$$
\frac{d}{d t} \phi_{4}(t, \widehat{\Omega})+c_{11} \Phi_{1}(t, \widehat{\Omega}) \leq c Y
$$

where

$$
\begin{aligned}
\phi_{4}(t, \widehat{\Omega})= & \phi_{3}(t, \widehat{\Omega})+\frac{\mu+\nu}{\varrho_{e}} \int_{\mathbb{R}^{2}} d \tau \int_{\widehat{\Omega}\left(\xi_{0}\right)} d z \frac{\left|\Delta^{2}(\tau) \widehat{\nabla}_{3} \widetilde{\eta}_{\sigma}\right|^{2}}{|\tau|^{2+2 \alpha}} \\
& +\frac{\mu+\nu}{\varrho_{e}} \int_{\mathbb{R}_{+}} d n \int_{\widehat{\Omega}\left(\xi_{0}\right)} d z \frac{\left|\Delta_{3}^{2}(n) \widehat{\nabla}_{3} \widetilde{\eta}_{\sigma}\right|^{2}}{|n|^{1+2 \alpha}} \\
& +\frac{1}{2} \int_{\mathbb{R}^{2}} d \tau \int_{\widehat{\Omega}\left(\xi_{0}\right)} d z\left(\widehat{\eta} \frac{\left|\Delta^{2}(\tau) \widetilde{u}_{z}\right|^{2}}{|\tau|^{2+2 \alpha}}+\frac{p_{1}}{\varrho_{e}} \frac{\left|\Delta^{2}(\tau) \widetilde{\eta}_{\sigma z}\right|^{2}}{|\tau|^{2+2 \alpha}}\right) \\
& +\frac{\sigma}{2} \int_{\mathbb{R}^{2}} d \tau \int_{\widehat{S}\left(\xi_{0}\right)} d z_{\widehat{S}} \frac{g_{\widehat{u}}^{\gamma \delta} \int_{0}^{t} \Delta^{2}(\tau) \widetilde{u}_{z_{\beta} z_{\gamma}} d t^{\prime} \cdot \widehat{n} \int_{0}^{t} \Delta^{2}(\tau) \widetilde{u}_{z_{\beta} z_{\delta}} d t^{\prime} \cdot \widehat{n}}{|\tau|^{2+2 \alpha}}
\end{aligned}
$$




$$
\begin{aligned}
& +\frac{\sigma}{2} \int_{\mathbb{R}^{2}} d \tau \int_{\widehat{S}\left(\xi_{0}\right)} d z_{\widehat{S}}\left(\frac{4 \Delta^{2}(\tau)\left(H(\cdot, 0)+2 / R_{2}\right) \widehat{\zeta}}{|\tau|^{1+\alpha}}\right. \\
& \left.+\frac{1}{4} \frac{\sum_{\gamma, \delta=1}^{2} \int_{0}^{t} \Delta^{2}(\tau) \widetilde{u}_{z_{\gamma} z_{\delta}} d t^{\prime} \cdot \widehat{n}}{|\tau|^{1+\alpha}}\right)^{2} \\
& -\frac{\sigma}{32} \int_{\mathbb{R}^{2}} d \tau \int_{\widehat{S}\left(\xi_{0}\right)} d z_{\widehat{S}} \frac{\sum_{\gamma, \delta=1}^{2}\left(\int_{0}^{t} \Delta^{2}(\tau) \widetilde{u}_{z_{\gamma} z_{\delta}} d t^{\prime}\right)^{2}}{|\tau|^{2+2 \alpha}}
\end{aligned}
$$

(3.63) $\Phi_{1}(t, \widehat{\Omega})=\Phi_{2}(t, \widehat{\Omega})+\int_{\mathbb{R}^{2}} d \tau \int_{\widehat{\Omega}\left(\xi_{0}\right)} d z \frac{\left|\Delta^{2}(\tau) \widehat{\nabla}_{3} \widehat{\eta}_{\sigma}\right|^{2}}{|\tau|^{2+2 \alpha}}$

$$
+\int_{\mathbb{R}^{2}} d \tau \int_{\widehat{\Omega}\left(\xi_{0}\right)} d z \frac{\left|\Delta^{2}(\tau) \widehat{\nabla}_{3}^{2} \widetilde{u}_{3}\right|^{2}}{|\tau|^{2+2 \alpha}}+\int_{\mathbb{R}_{+}} d n \int_{\widehat{\Omega}\left(\xi_{0}\right)} d z \frac{\left|\Delta_{3}^{2}(n) \widetilde{u}_{z z^{\prime}}\right|^{2}}{|n|^{1+2 \alpha}}
$$$$
+\int_{\mathbb{R}_{+}} d n \int_{\widehat{\Omega}\left(\xi_{0}\right)} d z \frac{\left|\Delta_{3}^{2}(n) \widehat{\nabla}_{3} \widetilde{\eta}_{\sigma}\right|^{2}}{|n|^{1+2 \alpha}}+\int_{\mathbb{R}_{+}} d n \int_{\widehat{\Omega}\left(\xi_{0}\right)} d z \frac{\left|\Delta_{3}^{2}(n) \widetilde{u}_{z_{3} z_{3}}\right|^{2}}{|n|^{1+2 \alpha}}
$$$$
+\int_{\mathbb{R}_{+}} d n \int_{\widehat{\Omega}\left(\xi_{0}\right)} d z \frac{\left|\Delta_{3}^{2}(n) \widetilde{\eta}_{\sigma z^{\prime}}\right|^{2}}{|n|^{1+2 \alpha}}+\mu \int_{\mathbb{R}^{2}} \frac{\left\|\Delta^{2}(\tau) \widetilde{u}\right\|_{2, \widehat{\Omega}}^{2}}{|\tau|^{2+2 \alpha}} d \tau
$$$$
+\int_{\mathbb{R}^{2}} \frac{\left\|\Delta^{2}(\tau) \widetilde{\eta}_{\sigma z}\right\|_{0, \widehat{\Omega}}^{2}}{|\tau|^{2+2 \alpha}} d \tau+\int_{\mathbb{R}^{2}} \frac{\left\|\Delta(\tau) \tilde{\vartheta}_{\sigma}\right\|_{2, \widehat{\Omega}}^{2}}{|\tau|^{2+2 \alpha}} d \tau
$$$$
+\int_{\mathbb{R}_{+}} d n \int_{\widehat{\Omega}\left(\xi_{0}\right)} d z \frac{\left|\Delta_{3}(n) \tilde{\vartheta}_{\sigma z_{3} z_{3}}\right|^{2}}{|n|^{1+2 \alpha}}+\int_{\mathbb{R}_{+}} d n \int_{\widehat{\Omega}\left(\xi_{0}\right)} d z \frac{\left|\Delta_{3}(n) \tilde{\vartheta}_{\sigma z z^{\prime}}\right|^{2}}{|n|^{1+2 \alpha}}
$$

$$
\begin{aligned}
Y= & \psi_{24}\left(a,\|F\|_{5 / 2+\alpha, \widehat{S}}\right)\left\{X+X_{2}\|u\|_{2+\alpha, \Omega^{t}}^{2}+\left\|\widetilde{u}_{t}\right\|_{\alpha, \widehat{\Omega}}^{2}+\left\|\vartheta_{\sigma t}\right\|_{\alpha, \widehat{\Omega}}^{2}\right. \\
& \left.+\varepsilon\left(\left\|H(\cdot, 0)+2 / R_{e}\right\|_{\alpha+1 / 2, S}^{2}+\|R(\cdot, t)-R(\cdot, 0)\|_{5 / 2+\alpha, S^{1}}^{2}\right)\right\}
\end{aligned}
$$

and $\phi_{3}, \Phi_{2}, X_{2}, X$ are given by (3.52), (3.53), (3.4), (3.54), respectively.

Now, using (2.9) and assuming that the $\delta$ from the local existence theorem (see Theorem 3.4 of [13]) such that $T^{1 / 2}\|u\|_{2+\alpha, \Omega^{T}} \leq \delta$ is sufficiently small we get

$$
\begin{aligned}
g_{\widehat{u}}^{\gamma \delta} \int_{0}^{t} \Delta^{2}(\tau) \widetilde{u}_{z_{\beta} z_{\gamma}} d t^{\prime} \cdot \widehat{n} \int_{0}^{t} \Delta^{2}(\tau) & \widetilde{u}_{z_{\beta} z_{\delta}} d t^{\prime} \cdot \widehat{n} \\
& \geq \frac{3}{4} \sum_{\gamma, \delta=1}^{2}\left(\int_{0}^{t} \Delta^{2}(\tau) \widetilde{u}_{z_{\gamma} z_{\delta}} d t^{\prime} \cdot \widehat{n}\right)^{2} .
\end{aligned}
$$


Moreover,

$$
\begin{aligned}
g_{\widehat{u}}^{\gamma \delta} \int_{0}^{t} \Delta^{3}(\tau) \frac{\partial \widetilde{u}}{\partial z_{\gamma}} d t^{\prime} \cdot \widehat{n} \int_{0}^{t} \Delta^{3}(\tau) \frac{\partial \widetilde{u}}{\partial z_{\delta}} & d t^{\prime} \cdot \widehat{n} \\
& \geq \frac{3}{4} \sum_{\gamma=1}^{2}\left(\int_{0}^{t} \Delta^{3}(\tau) \frac{\partial \widetilde{u}}{\partial z_{\gamma}} d t^{\prime} \cdot \widehat{n}\right)^{2} .
\end{aligned}
$$

Therefore integrating (3.62) with respect to time and using (3.65), (3.66), $(3,48)$ we obtain inequality $(3.40)$ for $t \leq T$.

This completes the proof of the lemma.

Now, similarly to the case of interior subdomains we will estimate the integrals $\int_{0}^{t}\left\|\widetilde{u}_{t^{\prime}}\right\|_{\alpha, \widehat{\Omega}}^{2} d t^{\prime}$ and $\int_{0}^{t}\left\|\widetilde{\vartheta}_{\sigma t^{\prime}}\right\|_{\alpha, \widehat{\Omega}}^{2} d t^{\prime}$. First, we use inequality (3.24). Then to estimate $\int_{\widehat{\Omega}}\left\|\widetilde{u}_{t^{\prime}}\right\|_{\alpha / 2,(0, t)}^{2} d z$ and $\int_{\widehat{\Omega}}\left\|\widetilde{\vartheta}_{\sigma t^{\prime}}\right\|_{\alpha / 2,(0, t)}^{2} d z$ we apply the method used to obtain (3.25) and (3.35). However, in this case we have to consider some additional terms which appear in connection with the boundary conditions $(2.7)_{4}$ and $(2.7)_{5}$.

LEMmA 3.9. Let the assumptions of Theorem 3.1 be satisfied. Then

$$
\begin{aligned}
& c_{12} \int_{\widehat{\Omega}}\left\|\widetilde{u}_{t^{\prime}}\right\|_{\alpha / 2,(0, t)}^{2} d z \\
& \quad+\left.\int_{0}^{h_{0}} \frac{d h}{h^{1+\alpha}} \int_{\widehat{\Omega}}\left(\frac{\mu}{4}\left|\mathbb{S}\left(\Delta_{t^{\prime}} \widetilde{u}\right)\right|^{2}+\frac{\nu-\mu}{2}\left|\operatorname{div} \Delta_{t^{\prime}} \widetilde{u}\right|^{2}\right) d z\right|_{t^{\prime}=t-h_{0}} \\
& \leq \varepsilon\left(\|u\|_{2+\alpha, \Omega^{t}}^{2}+\left\|\vartheta_{\sigma}\right\|_{2+\alpha, \Omega^{t}}^{2}+\left\|\eta_{\sigma}\right\|_{1+\alpha, \Omega^{t}}^{2}\right)+c(\varepsilon, t)\left[d\left\|\widetilde{\vartheta}_{\sigma}\right\|_{2+\alpha, \widehat{\Omega}^{t}}^{2}\right. \\
& \left.\quad+\|u\|_{0, \Omega^{t}}^{2}+\left\|\vartheta_{\sigma}\right\|_{0, \Omega^{t}}^{2}+\|k\|_{0, \Omega^{t}}^{2}+\left(1+X_{3}\right) X_{3}\left(X_{3}+X_{4}\right)\right],
\end{aligned}
$$

where $t \leq T, c_{12}>0$ is a constant, $c(\varepsilon, t)$ is a positive continuous function nondecreasing with respect to $t$, and $X_{3}$ and $X_{4}$ are given by (3.38) and (3.39), respectively.

Proof. The calculations of Lemma 3.4 and the boundary condition $(2.7)_{4}$ yield

$$
\begin{aligned}
& \int_{0}^{h_{0}} \frac{d h}{h^{1+\alpha}} \int_{0}^{t-h_{0}} \psi\left(t^{\prime}\right) d t^{\prime} \int_{\widehat{\Omega}} \widehat{\eta}\left|\Delta_{t^{\prime}} \widetilde{u}_{t^{\prime}}\right|^{2} d z \\
& \quad+\left.\int_{0}^{h_{0}} \frac{d h}{h^{1+\alpha}} \int_{\widehat{\Omega}}\left(\frac{\mu}{4}\left|\mathbb{S}\left(\Delta_{t^{\prime}} \widetilde{u}\right)\right|^{2}+\frac{\nu-\mu}{2}\left|\operatorname{div} \Delta_{t^{\prime}} \widetilde{u}\right|^{2}\right) d z\right|_{t^{\prime}=t-h_{0}} \\
& \leq I+\sum_{i=1}^{5}\left|K_{i}\right|
\end{aligned}
$$


where

$$
\begin{aligned}
(3.69) & I \leq \varepsilon\left(\|u\|_{2+\alpha, \Omega^{t}}^{2}+\left\|\vartheta_{\sigma}\right\|_{2+\alpha, \Omega^{t}}^{2}+\left\|\eta_{\sigma}\right\|_{1+\alpha, \Omega^{t}}^{2}\right) \\
& +d\left\|\widetilde{\vartheta}_{\sigma}\right\|_{2+\alpha, \widehat{\Omega}^{t}}^{2}+c(\varepsilon, t)\left[\|u\|_{0, \Omega^{t}}^{2}+\left\|\vartheta_{\sigma}\right\|_{0, \Omega^{t}}^{2}\right. \\
& +\left\|\eta_{\sigma}\right\|_{0, \Omega^{t}}^{2}+\|k\|_{0, \Omega^{t}}^{2}+\|u\|_{2+\alpha, \Omega^{t}}^{2} \sup _{0 \leq t^{\prime} \leq t}\|u\|_{1+\alpha, \Omega}^{2} \\
& \left.+\|\widehat{u}\|_{2+\alpha, \widehat{\Omega}^{t}}^{2}\|\widehat{u}\|_{2+\alpha, \hat{\Omega}^{t}}^{2}+\left\|\widehat{\vartheta}_{\sigma}\right\|_{2+\alpha, \widehat{\Omega}^{t}}^{2}+\left\|\eta_{\sigma}\right\|_{1+\alpha, \Omega^{t}}^{2}\right)\left(1+\|\widehat{u}\|_{1+\alpha, \widehat{\Omega}^{t}}^{2}\right) \\
& +\sup _{0 \leq t^{t} \leq t}\|\widehat{u}\|_{1+\alpha, \widehat{\Omega}^{2}}^{2}\left(\left\|\widehat{\vartheta}_{\sigma}\right\|_{2+\alpha, \widehat{\Omega}^{t}}^{2}+\left\|\widehat{\eta}_{\sigma}\right\|_{1+\alpha, \widehat{\Omega}^{t}}^{2}\right) \\
& +\left\|\widehat{\vartheta}_{\sigma}\right\|_{2+\alpha, \widehat{\Omega}^{t}}^{2}\left(\left\|\widetilde{\vartheta}_{\sigma}\right\|_{1+\alpha, \widehat{\Omega}^{t}}^{2}+\left\|\widetilde{\eta}_{\sigma}\right\|_{1+\alpha, \widehat{\Omega}^{t}}^{2}\right) \\
& \left.+\| \widehat{u}_{2+\alpha, \widehat{\Omega}^{t}}^{2}\left(\sup _{0 \leq t^{t} \leq t}\left\|\widetilde{\eta}_{\sigma}\right\|_{1+\alpha, \widehat{\Omega}^{2}}^{2}+\sup _{0 \leq t^{\prime} \leq t}\left\|\widetilde{\vartheta}_{\sigma}\right\|_{1+\alpha, \widehat{\Omega}}^{2}\right)\right] .
\end{aligned}
$$

and

$$
K_{1}=\int_{0}^{h_{0}} \frac{d h}{h^{1+\alpha}} \int_{0}^{t-h_{0}} \psi\left(t^{\prime}\right) d t^{\prime} \int_{\widehat{S}} \Delta_{t^{\prime}} \widetilde{p}_{\sigma} I \widehat{n}^{\prime} \Delta_{t^{\prime}} \widetilde{u}_{t^{\prime}} d z_{\widehat{S}}
$$$$
K_{2}=-\sigma \int_{0}^{h_{0}} \frac{d h}{h^{1+\alpha}} \int_{0}^{t-h_{0}} \psi\left(t^{\prime}\right) d t^{\prime} \int_{\widehat{S}} \Delta_{t^{\prime}}\left(\Delta_{\widehat{S}}\right) \int_{0}^{t^{\prime}} \widetilde{u} d \tau \cdot \Delta_{t^{\prime}} \widetilde{u}_{t^{\prime}} d z_{\widehat{S}}
$$

3.72) $K_{3}=-\sigma \int_{0}^{h_{0}} \frac{d h}{h^{1+\alpha}} \int_{0}^{t-h_{0}} \psi\left(t^{\prime}\right) d t^{\prime} \Delta_{\widehat{S}} \Delta_{t^{\prime}} \int_{0}^{t^{\prime}} \widetilde{u} d \tau \cdot \Delta_{t^{\prime}} \widetilde{u}_{t^{\prime}} d z_{\widehat{S}}$,

(3.73) $\quad K_{4}=\int_{0}^{h_{0}} \frac{d h}{h^{1+\alpha}} \int_{0}^{t-h_{0}} \psi\left(t^{\prime}\right) d t^{\prime} \int_{\widehat{S}} \widehat{\mathbb{T}}\left(\widetilde{u}, \widetilde{p}_{\sigma}\right) \Delta_{t^{\prime}} \widehat{n} \cdot \Delta_{t^{\prime}} \widetilde{u}_{t} d z_{\widehat{S}}$.

Notice that the sum of the first two terms on the right-hand side of (3.68) has the same form as the right-hand side of (3.25). Thus, it remains to estimate $K_{i}(i=1, \ldots, 5)$.

First, we have

$$
\begin{aligned}
\left|K_{1}\right| \leq & \varepsilon \int_{\widehat{S}} d z_{\widehat{S}} \int_{0}^{h_{0}} d h \int_{0}^{t-h_{0}} d t^{\prime} \frac{\left|\Delta_{t^{\prime}} \widetilde{u}_{t^{\prime}}\right|^{2}}{h^{1+2(\alpha / 2-1 / 4)}} \\
& +c(\varepsilon) \int_{\widehat{S}} d z_{\widehat{S}} \int_{0}^{h_{0}} d h \int_{0}^{t-h_{0}} d t^{\prime} \frac{\left|\Delta_{t^{\prime}}\left(p_{1} \widetilde{\eta}_{\sigma}+p_{2} \widetilde{\vartheta}_{\sigma}\right)\right|^{2}}{h^{3 / 2+\alpha}} \\
\leq & \varepsilon\|\widetilde{u}\|_{3 / 2+\alpha, \widehat{S}^{t}}^{2}+c(\varepsilon) \int_{\widehat{S}} d z_{\widehat{S}} \int_{0}^{h_{0}} d h \int_{0}^{t-h_{0}} d t^{\prime} \frac{\left|\Delta_{t^{\prime}} p_{1} \widetilde{\eta}_{\sigma}\right|^{2}}{h^{3 / 2+\alpha}}
\end{aligned}
$$




$$
\begin{aligned}
& +c(\varepsilon) \int_{\widehat{S}} d z_{\widehat{S}} \int_{0}^{h_{0}} d h \int_{0}^{t-h_{0}} d t^{\prime} \frac{\left|\Delta_{t^{\prime}} p_{2} \widetilde{\vartheta}_{\sigma}\right|^{2}}{h^{3 / 2+\alpha}}+c(\varepsilon) \int_{\widehat{S}} d z_{\widehat{S}} \int_{0}^{h_{0}} d h \int_{0}^{t-h_{0}} d t^{\prime} \frac{\left|\Delta_{t} \widetilde{\eta}_{\sigma}\right|^{2}}{h^{3 / 2+\alpha}} \\
& +c(\varepsilon) \int_{\widehat{S}} d z_{\widehat{S}} \int_{0}^{h_{0}} d h \int_{0} d t^{\prime} \frac{\left|\Delta_{t} \widetilde{\vartheta}_{\sigma}\right|^{2}}{h^{3 / 2+\alpha}} \equiv \varepsilon\|\widetilde{u}\|_{2+\alpha, \widehat{\Omega}^{t}}^{2}+\sum_{i=1}^{4} K_{1}^{i} .
\end{aligned}
$$

We have to estimate the terms $K_{1}^{i}(i=1, \ldots, 4)$. First, we obtain

$$
\begin{aligned}
K_{1}^{4} & \leq c(\varepsilon) \int_{\widehat{S}} d z_{\widehat{S}} \int_{0}^{h_{0}} d h \int_{0}^{t-h_{0}} d t^{\prime} \frac{\left|\int_{t^{\prime}}^{t^{\prime}+h} \widetilde{\vartheta}_{\sigma \tau} d \tau\right|^{2}}{h^{3 / 2+\alpha}} \\
& \leq c(\varepsilon) h_{0}^{3 / 2-\alpha} \int_{0}^{t-h_{0}} d t^{\prime} \int_{\widehat{S}} d z_{\widehat{S}}\left(\sup _{h>0} \frac{1}{h} \int_{t^{\prime}}^{t^{\prime}+h}\left|\widetilde{\vartheta}_{\sigma \tau}\right| d \tau\right)^{2} \\
& \leq c(\varepsilon) t^{3 / 2-\alpha} \int_{0}^{t}\left\|\widetilde{\vartheta}_{\sigma t^{\prime}}\right\|_{0, \widehat{S}}^{2} d t^{\prime} \\
& \leq c(\varepsilon) t^{3 / 2-\alpha}\left(\varepsilon_{1}^{1-\varkappa} \int_{0}^{t}\left\|\widetilde{\vartheta}_{\sigma t^{\prime}}\right\|_{\alpha, \widehat{\Omega}}^{2} d t^{\prime}+c \varepsilon_{1}^{-\varkappa} \int_{0}^{t}\left\|\widetilde{\vartheta}_{\sigma t^{\prime}}\right\|_{0, \widehat{\Omega}}^{2} d t^{\prime}\right) .
\end{aligned}
$$

From equation $(2.7)_{3}$ we calculate

$$
\begin{aligned}
\left\|\widetilde{\vartheta}_{\sigma t}\right\|_{0, \widehat{\Omega}}^{2} & \leq \varepsilon_{2}^{1-\varkappa_{2}}\left(\|u\|_{2+\alpha, \Omega}^{2}+\left\|\vartheta_{\sigma}\right\|_{2+\alpha, \Omega}^{2}\right)+d\left\|\widetilde{\vartheta}_{\sigma}\right\|_{2+\alpha, \widehat{\Omega}}^{2} \\
& +c \varepsilon_{2}^{-\varkappa_{2}}\left[\|u\|_{0, \Omega}^{2}+\left\|\vartheta_{\sigma}\right\|_{0, \Omega}^{2}+\|k\|_{0, \Omega}^{2}\right. \\
& +\|\widehat{u}\|_{1+\alpha, \widehat{\Omega}}^{2}\left(\|\widehat{u}\|_{1+\alpha, \widehat{\Omega}}^{2}+\left\|\widehat{\vartheta}_{\sigma}\right\|_{\alpha, \widehat{\Omega}}^{2}+\left\|\widehat{\eta}_{\sigma}\right\|_{\alpha, \widehat{\Omega}}^{2}+\|\widehat{u}\|_{1+\alpha, \widehat{\Omega}^{t}}^{2}\right) \\
& \left.+\|\widehat{u}\|_{2+\alpha, \widehat{\Omega}^{t}}^{2}\left\|\widetilde{\vartheta}_{\sigma}\right\|_{2, \widehat{\Omega}}^{2}\right] .
\end{aligned}
$$

Using (3.76) in (3.75) with appropriate choice of $\varepsilon_{1}$ and $\varepsilon_{2}$ yields

$$
\begin{aligned}
K_{1}^{4} \leq & \varepsilon\left(\|u\|_{2+\alpha, \Omega^{t}}^{2}+\left\|\vartheta_{\sigma}\right\|_{2+\alpha, \Omega^{t}}^{2}\right) \\
& +c(\varepsilon, t)\left(d\left\|\widetilde{\vartheta}_{\sigma}\right\|_{2+\alpha, \widehat{\Omega}^{t}}^{2}+\|u\|_{0, \Omega^{t}}^{2}+\left\|\vartheta_{\sigma}\right\|_{0, \Omega^{t}}^{2}+\|k\|_{0, \Omega^{t}}^{2}\right. \\
& +\sup _{0 \leq t^{\prime} \leq t}\|\widehat{u}\|_{1+\alpha, \widehat{\Omega}^{2}}^{2}\left(\|\widehat{u}\|_{1+\alpha, \widehat{\Omega}^{t}}^{2}+\left\|\widehat{\vartheta}_{\sigma}\right\|_{\alpha, \widehat{\Omega}^{t}}^{2}+\left\|\widehat{\eta}_{\sigma}\right\|_{\alpha, \widehat{\Omega}^{t}}^{2}\right) \\
& \left.+\|\widehat{u}\|_{2+\alpha, \widehat{\Omega}^{t}}^{2}\left\|\widetilde{\vartheta}_{\sigma}\right\|_{2, \widehat{\Omega}^{t}}^{2}\right],
\end{aligned}
$$

where $c(\varepsilon, t)$ is a positive continuous function increasing with respect to $t$. Now, we estimate

$$
K_{1}^{3} \leq c(\varepsilon) \int_{\widehat{S}} d z_{\widehat{S}} \int_{0}^{h_{0}} d h \int_{0}^{t-h_{0}} d t^{\prime} \frac{\left|\int_{t^{\prime}}^{t^{\prime}+h} \widehat{u}_{z} d \tau\right|^{2}}{h^{3 / 2+\alpha}}
$$




$$
\begin{aligned}
& \leq c(\varepsilon) h_{0}^{3 / 2-\alpha} \int_{0}^{t-h_{0}} d t^{\prime} \int_{\widehat{S}} d z_{\widehat{S}}\left(\sup _{h>0} \frac{1}{h} \int_{t^{\prime}}^{t^{\prime}+h}\left|\widehat{u}_{z}\right| d \tau\right)^{2} \\
& \leq c(\varepsilon) t^{3 / 2-\alpha} \int_{0}^{t}\left\|\widehat{u}_{z}\right\|_{0, \widehat{S}^{2}}^{2} d t^{\prime} \leq c(\varepsilon) t^{3 / 2-\alpha}\left(\varepsilon_{1}^{1-\varkappa}\|\widehat{u}\|_{2+\alpha, \widehat{\Omega}^{t}}^{2}+c \varepsilon_{1}^{-\varkappa}\|\widehat{u}\|_{0, \widehat{\Omega}^{t}}^{2}\right) \\
& \leq \varepsilon\|\widehat{u}\|_{2+\alpha, \widehat{\Omega}^{t}}^{2}+c(\varepsilon, t)\|\widehat{u}\|_{0, \widehat{\Omega}^{t}}^{2},
\end{aligned}
$$

where we assumed $\varepsilon_{1}=\left(\varepsilon /\left(c(\varepsilon) t^{3 / 2-\alpha}\right)\right)^{1 /(1-\varkappa)}$.

Next, we have

$$
\begin{aligned}
K_{1}^{1} \leq & c(\varepsilon) \int_{\widehat{S}} d z \int_{\widehat{S}} \int_{0}^{h_{0}} d h \int_{0}^{t-h_{0}} d t^{\prime} \frac{\left|\Delta_{t^{\prime}} \widehat{\eta}_{\sigma}\right|^{2}\left|\widetilde{\eta}_{\sigma}\right|^{2}}{h^{3 / 2+\alpha}} \\
& +c(\varepsilon) \int_{\widehat{S}} d z \int_{\widehat{S}} \int_{0}^{h_{0}} d h \int_{0}^{t-h_{0}} d t^{\prime} \frac{\left|\Delta_{t^{\prime}} \widehat{\vartheta}_{\sigma}\right|^{2}\left|\widetilde{\eta}_{\sigma}\right|^{2}}{h^{3 / 2+\alpha}} \\
\leq & c(\varepsilon) \sup _{0 \leq t^{\prime} \leq t}\left\|\widetilde{\eta}_{\sigma}\right\|_{1+\alpha, \widehat{\Omega}}^{2}\left(\left|K_{1}^{4}\right|+\left|K_{1}^{3}\right|\right) \\
\leq & c(\varepsilon) t^{3 / 2-\alpha} \sup _{0 \leq t^{\prime} \leq t}\left\|\widetilde{\eta}_{\sigma}\right\|_{1+\alpha, \widehat{\Omega}^{2}}^{2}\left(\left\|\widetilde{\vartheta}_{\sigma}\right\|_{2+\alpha, \widehat{\Omega}^{t}}^{2}+\|\widehat{u}\|_{2+\alpha, \widehat{\Omega}^{t}}^{2}\right) .
\end{aligned}
$$

In the same way we estimate $\left|K_{1}^{2}\right|$.

Thus, taking into account (3.74)-(3.79) we get

$$
\begin{aligned}
\left|K_{1}\right| \leq & \varepsilon\left(\|u\|_{2+\alpha, \Omega^{t}}^{2}+\left\|\vartheta_{\sigma}\right\|_{2+\alpha, \Omega^{t}}^{2}\right)+c(\varepsilon, t)\left[d\left\|\widetilde{\vartheta}_{\sigma}\right\|_{2+\alpha, \widehat{\Omega}^{t}}^{2}\right. \\
& +\|u\|_{0, \Omega^{t}}^{2}+\left\|\vartheta_{\sigma}\right\|_{0, \Omega^{t}}^{2}+\|k\|_{0, \Omega^{t}}^{2}+\sup _{0 \leq t^{\prime} \leq t}\|\widehat{u}\|_{1+\alpha, \widehat{\Omega}}^{2}\left(\|\widehat{u}\|_{1+\alpha, \widehat{\Omega}^{t}}^{2}\right. \\
& \left.+\left\|\widehat{\vartheta}_{\sigma}\right\|_{\alpha, \widehat{\Omega}^{t}}^{2}+\left\|\widehat{\eta}_{\sigma}\right\|_{\alpha, \widehat{\Omega}^{t}}^{2}\right)+\|\widehat{u}\|_{2+\alpha, \widehat{\Omega}^{t}}^{2}\left\|\widetilde{\vartheta}_{\sigma}\right\|_{2, \widehat{\Omega}^{t}}^{2} \\
& \left.+\sup _{0 \leq t^{\prime} \leq t}\left\|\widetilde{\eta}_{\sigma}\right\|_{1+\alpha, \widehat{\Omega}^{2}}^{2}\left(\left\|\widetilde{\vartheta}_{\sigma}\right\|_{2+\alpha, \widehat{\Omega}^{t}}^{2}+\|\widehat{u}\|_{2+\alpha, \widehat{\Omega}^{t}}^{2}\right)\right] .
\end{aligned}
$$

In the same way we estimate $K_{2}, K_{3}, K_{4}$ obtaining

$$
\begin{aligned}
\left|K_{2}\right| & \leq \varepsilon\|\widetilde{u}\|_{2+\alpha, \widehat{\Omega}^{t}}^{2}+c(\varepsilon) t^{3 / 2-\alpha}\|\widetilde{u}\|_{2+\alpha, \Omega^{t}}^{4}\left(1+\|\widetilde{u}\|_{2+\alpha, \widehat{\Omega}^{t}}^{2}\right), \\
\left|K_{3}\right| & \leq \varepsilon\|\widetilde{u}\|_{2+\alpha, \widehat{\Omega}^{t}}^{2}+c(\varepsilon) t^{3 / 2-\alpha}\left(\|\widehat{u}\|_{2+\alpha, \Omega^{t}}^{2}\|\widetilde{u}\|_{2+\alpha, \widehat{\Omega}^{t}}^{2}+\|\widetilde{u}\|_{0, \widehat{\Omega}^{t}}^{2}\right) \\
\left|K_{4}\right| & \leq \varepsilon\|\widetilde{u}\|_{2+\alpha, \widehat{\Omega}^{t}}^{2}+c(\varepsilon) t^{3 / 2-\alpha}\left(\sup _{0 \leq t^{\prime} \leq t}\|\widetilde{u}\|_{1+\alpha, \widehat{\Omega}}^{2}\right. \\
& \left.+\sup _{0 \leq t^{\prime} \leq t}\left\|\widetilde{\eta}_{\sigma}\right\|_{\alpha, \widehat{\Omega}^{2}}^{2}+\sup _{0 \leq t^{\prime} \leq t}\left\|\widetilde{\vartheta}_{\sigma}\right\|_{\alpha, \widehat{\Omega}}^{2}\right)\|\widehat{u}\|_{2+\alpha, \widehat{\Omega}^{t}}^{2}\left(1+\|\widehat{u}\|_{2+\alpha, \widehat{\Omega}^{t}}^{2}\right) .
\end{aligned}
$$

Now, inequalities (3.68), (3.69), (3.74), (3.80)-(3.83) yield (3.67).

This completes the proof of the lemma. 
The lemma below can be proved in the same way as Lemma 3.9.

Lemma 3.10. Let the assumptions of Theorem 3.1 be satisfied. Then

$$
\begin{aligned}
& c_{13} \int_{\widehat{\Omega}}\left\|\widetilde{\vartheta}_{\sigma t^{\prime}}\right\|_{\alpha / 2,(0, t)}^{2} d z+\left.\frac{\varkappa}{2} \int_{0}^{h_{0}} \frac{d h}{h^{1+\alpha}} \int_{\widehat{\Omega}}\left(\left|\nabla\left(\Delta_{t^{\prime}} \widetilde{\vartheta}_{\sigma}\right)\right|^{2}\right)\right|_{t^{\prime}=t-h_{0}} d z \\
& \leq \varepsilon\left(\left\|\vartheta_{\sigma}\right\|_{2+\alpha, \Omega^{t}}^{2}+\|u\|_{2+\alpha, \Omega^{t}}^{2}+\left\|\eta_{\sigma}\right\|_{1+\alpha, \Omega^{t}}^{2}\right) \\
&+d\left\|\widetilde{\vartheta}_{\sigma}\right\|_{2+\alpha, \widehat{\Omega}^{t}}^{2}+c(\varepsilon, t)\left[\|u\|_{0, \Omega^{t}}^{2}+\left\|\vartheta_{\sigma}\right\|_{0, \Omega^{t}}^{2}\right. \\
&+\left\|\eta_{\sigma}\right\|_{0, \Omega^{t}}^{2}+\|k\|_{\alpha, \Omega^{t}}^{2}+\|\bar{\vartheta}\|_{\alpha+1 / 2, S^{t}}^{2} \\
&+\|\widehat{u}\|_{2+\alpha, \hat{\Omega}^{t}}^{2}\|\widehat{u}\|_{2+\alpha, \widehat{\Omega}^{t}}^{2}+\sup _{0 \leq t^{\prime} \leq t}\left\|\widehat{\vartheta}_{\sigma}\right\|_{1+\alpha, \widehat{\Omega}}^{2} \\
&+\sup _{0 \leq t^{\prime} \leq t}\left\|\widehat{\eta}_{\sigma}\right\|_{1+\alpha, \widehat{\Omega}}^{2}+\sup _{0 \leq t^{\prime} \leq t}\|\widehat{u}\|_{1+\alpha, \widehat{\Omega}}^{2} \\
&+\|\widehat{u}\|_{1+\alpha, \widehat{\Omega}^{t}}^{2} \sup _{0 \leq t^{\prime} \leq t}\|\widehat{u}\|_{1+\alpha, \widehat{\Omega}}^{2}+\left\|\widetilde{\vartheta}_{\sigma}\right\|_{2+\alpha, \widehat{\Omega}^{t}}^{2} \\
&\left.+\|\widehat{u}\|_{2+\alpha, \widehat{\Omega}^{t}}^{2}\left\|\widetilde{\vartheta}_{\sigma}\right\|_{2+\alpha, \widehat{\Omega}^{t}}^{2}\right)+\|u\|_{2+\alpha, \Omega^{t}}^{2}\|u\|_{1+\alpha, \Omega^{t}}^{2} \\
&\left.+\sup _{0 \leq t^{\prime} \leq t}\|\widehat{\eta}\|_{1+\alpha, \widehat{\Omega}}^{2}\|\widetilde{k}\|_{\alpha, \widehat{\Omega}^{t}}^{2}+\left\|\widehat{\vartheta}_{\sigma}\right\|_{2+\alpha, \widehat{\Omega}^{t}}^{2}\left\|\widetilde{\vartheta}_{\sigma}\right\|_{2+\alpha, \widehat{\Omega}^{t}}^{2}\right]
\end{aligned}
$$

where $t \leq T, c_{13}>0$ is a constant, and $c(\varepsilon, t)$ is a positive continuous function nondecreasing with respect to $t$.

REMARK 3.11. As in the proofs of Lemmas 3.4 and 3.6, we had to derive inequalities (3.67) and (3.84) in such a way as to obtain functions $c(\varepsilon, t)$ on the right-hand sides nondecreasing with respect to $t$.

Now, we can finish the proof of Theorem 3.1.

Proof of Theorem 3.1. In addition to the estimates derived above we need one more estimate for $\int_{\Omega}\left\|\eta_{\sigma t^{\prime}}\right\|_{\alpha / 2,(0, t)}^{2} d \xi$, where $t \leq T$.

Using $(1.6)_{2}$ and (3.31) we get

$$
\begin{aligned}
\int_{\Omega}\left\|\eta_{\sigma t^{\prime}}\right\|_{\alpha / 2,(0, t)}^{2} d \xi & \\
\leq & \varepsilon\left(\|u\|_{2+\alpha, \Omega^{t}}^{2}+\int_{0}^{t}\left\|\eta_{\sigma}\right\|_{1+\alpha, \Omega}^{2} d t^{\prime}+\int_{0}^{t}\left\|\vartheta_{\sigma}\right\|_{1+\alpha, \Omega}^{2} d t^{\prime}\right) \\
& +c(\varepsilon, t)\left(\|u\|_{0, \Omega^{t}}^{2}+\left\|\vartheta_{\sigma}\right\|_{0, \Omega^{t}}^{2}+\left\|\eta_{\sigma}\right\|_{0, \Omega^{t}}^{2}+\|u\|_{2+\alpha, \Omega^{t}}^{4}\right) .
\end{aligned}
$$

Now let us introduce a partition of unity $\left(\left\{\widetilde{\Omega}_{i}\right\},\left\{\zeta_{i}\right\}\right), \Omega \subset \bigcup_{i \in \mathfrak{M} \cup \mathfrak{N}} \widetilde{\Omega}_{i}$, such that $\widetilde{\Omega}_{i}, i \in \mathfrak{M}$, is an interior subdomain, and $\widetilde{\Omega}_{i}, i \in \mathfrak{N}$, is a boundary 
subdomain. Then we introduce the quantity

$$
\phi(t, \Omega)=\sum_{i \in \mathfrak{M}} \phi_{1}\left(t, \widetilde{\Omega}_{i}\right)+\sum_{i \in \mathfrak{N}} \phi_{2}\left(t, \widehat{\Omega}_{i}\right),
$$

where $\widehat{\Omega}_{i}=\Phi\left(\widetilde{\Omega}_{i}\right)$ is given by $(2.4), \phi_{1}$ and $\phi_{2}$ are given by (3.36) and (3.41), respectively.

Now taking into account estimates (3.85), (3.37), (3.40), (3.67) and (3.84) (where in all terms of (3.40), (3.67), (3.84) except $\phi_{2}$ and $\phi_{5}$ we return to the old variables $\xi$ ) we obtain (3.1).

This completes the proof of the theorem.

4. A remark on an estimate derived in [14]. In [14] we obtained an estimate which showed the increase of the regularity of the solution. Since we omitted $r$ and $\bar{\theta}$ in our considerations, the estimate derived in [14] had the form

$$
\sup _{t_{0}+\lambda \leq t \leq T}\|u\|_{2+\alpha, \Omega}^{2}+\sup _{t_{0}+\lambda \leq t \leq T}\left\|\vartheta_{\sigma}\right\|_{2+\alpha, \Omega}^{2} \leq c(K) \bar{K},
$$

where $\bar{K}=\|n\|_{2+\alpha, \Omega^{T}}^{2}+\left\|\vartheta_{\sigma}\right\|_{2+\alpha, \Omega^{T}}^{2}$,

$$
\begin{gathered}
K=\bar{K}+\sup _{0 \leq t \leq T}\|u\|_{1+\alpha, \Omega}^{2}+\sup _{0 \leq t \leq T}\left\|\vartheta_{\sigma}\right\|_{1+\alpha, \Omega}^{2} \\
+\left\|\eta_{\sigma}\right\|_{1+\alpha, \Omega^{T}}^{2}+\sup _{0 \leq t \leq T}\left\|\eta_{\sigma}\right\|_{1+\alpha, \Omega}^{2} ;
\end{gathered}
$$

$c(K)$ is a positive nondecreasing function of $K$ depending also on $T$.

To obtain the analogous inequality for nonvanishing $r$ and $\bar{\theta}$ we have to estimate additionally $\left\|\eta^{\prime} k^{(s)}\right\|_{\alpha, Q_{\lambda / 2}}^{2},\left\|\eta_{*}^{(s)} k_{\lambda}\right\|_{\alpha, Q_{\lambda / 2}}^{2}$ and $\left\|\bar{\vartheta}^{(s)}\right\|_{\alpha+1 / 2, G_{\lambda / 2}}^{2}$ (see [14]), where $\eta^{\prime}(\xi, t)=\eta(\xi, t-s), k^{(s)}(\xi, t)=k_{\lambda}(\xi, t)-k_{\lambda}(\xi, t-s)$, $\eta_{*}^{(s)}(\xi, t)=\eta(\xi, t)-\eta(\xi, t-s), \bar{\vartheta}^{(s)}(\xi, t)=\bar{\vartheta}_{\lambda}(\xi, t)-\bar{\vartheta}_{\lambda}(\xi, t-s), k_{\lambda}=\zeta_{\lambda} k$, $\bar{\vartheta}_{\lambda}=\zeta_{\lambda} \bar{\vartheta} ; \zeta_{\lambda} \in C^{\infty}$ is a function such that $\zeta_{\lambda}(t)=1$ for $t \geq t_{0}+\lambda, \zeta_{\lambda}(t)=0$ for $t \leq t_{0}+\lambda / 2 ; Q_{\lambda / 2}=\Omega \times\left(t_{0}+\lambda / 2, T\right), G_{\lambda / 2}=S \times\left(t_{0}+\lambda / 2, T\right)$, $0<s<t_{0}$.

By simple calculations we get

$$
\begin{aligned}
\left\|\eta^{\prime} k^{(s)}\right\|_{\alpha, Q_{\lambda / 2}}^{2} & +\left\|\eta_{*}^{(s)} k_{\lambda}\right\|_{\alpha, Q_{\lambda / 2}}^{2} \\
\leq & c\left(\int_{t_{0}+\lambda / 2}^{T}\left\|\eta^{\prime} k^{(s)}\right\|_{1, \Omega}^{2} d t+\int_{\Omega}\left\|\eta^{\prime} k^{(s)}\right\|_{1,\left(t_{0}+\lambda / 2, T\right)}^{2} d \xi\right. \\
& \left.+\int_{t_{0}+\lambda / 2}^{T}\left\|\eta_{*}^{(s)} k_{\lambda}\right\|_{1, \Omega}^{2} d t+\int_{\Omega}\left\|\eta_{*}^{(s)} k_{\lambda}\right\|_{1,\left(t_{0}+\lambda / 2, T\right)}^{2} d \xi\right) \\
\leq & c(K) s^{1+\alpha}\|r\|_{C_{B}^{2}\left(\mathbb{R}^{3} \times \mathbb{R}_{+}\right)}^{2}
\end{aligned}
$$


and

$$
\begin{aligned}
\left\|\bar{\vartheta}^{(s)}\right\|_{\alpha+1 / 2, G_{\lambda / 2}}^{2} & \leq c\left\|\bar{\vartheta}^{(s)}\right\|_{\alpha+1, Q_{\lambda / 2}}^{2} \\
& \leq c\left(\int_{t_{0}+\lambda / 2}^{T}\left\|\bar{\vartheta}^{(s)}\right\|_{2, \Omega}^{2} d t+\int_{\Omega}\left\|\bar{\vartheta}^{(s)}\right\|_{1,\left(t_{0}+\lambda / 2, T\right)}^{2} d \xi\right) \\
& \leq c(K) s^{1+\alpha}\|\bar{\theta}\|_{C_{B}^{3}\left(\mathbb{R}^{3} \times \mathbb{R}_{+}\right)}^{2} .
\end{aligned}
$$

Taking into account (4.2)-(4.3) and the considerations from [14] we obtain

TheOREM 4.1. Let $(u, \vartheta, \eta) \in W_{2}^{2+\alpha, 1+\alpha / 2}\left(\Omega^{T}\right) \times W_{2}^{2+\alpha, 1+\alpha / 2}\left(\Omega^{T}\right) \times$ $C\left([0, T] ; W_{2}^{2+\alpha}(\Omega)\right) \cap W_{2}^{1+\alpha, 1 / 2+\alpha / 2}\left(\Omega^{T}\right)(\alpha \in(3 / 4,1))$ be the local solution of problem (1.1). Then for any $0<t_{0}<T$ and $\lambda>0$ the function $u$ is in $C\left(\left[t_{0}+\lambda, T\right] ; W_{2}^{2+\alpha}(\Omega)\right)$ and

$$
\begin{aligned}
\sup _{t_{0}+\lambda \leq t \leq T}\|u\|_{2+\alpha, \Omega}^{2} & +\sup _{t_{0}+\lambda \leq t \leq T}\left\|\vartheta_{\sigma}\right\|_{2+\alpha, \Omega}^{2} \\
& \leq c(K)\left(\bar{K}+\|r\|_{C_{B}^{2}\left(\mathbb{R}^{3} \times \mathbb{R}_{+}\right)}+\|\bar{\theta}\|_{C_{B}^{3}\left(\mathbb{R}^{3} \times \mathbb{R}_{+}\right)}^{2}\right) .
\end{aligned}
$$

REMARK 4.2. By Theorem 4.1 the global existence theorem proved in [12] holds for $r$ and $\bar{\theta}$ satisfying assumption (1.7).

\section{References}

[1] R. A. Adams, Sobolev Spaces, Academic Press, New York, 1975.

[2] S. Agmon, A. Douglis and L. Nirenberg, Estimates near the boundary for solutions of elliptic partial differential equations satisfying general boundary conditions, I, II, Comm. Pure Appl. Math. 12 (1959), 623-727; 17 (1964), 35-92.

[3] O. V. Besov, V. P. II'in and S. M. Nikol'skiu, Integral Representations of Functions and Imbedding Theorems, Nauka, Moscow 1975 (in Russian); English transl.: Scripta Series in Mathematics, Winston and Halsted Press, 1979.

[4] K. K. Golovkin, On equivalent norms for fractional spaces, Trudy. Mat. Inst. Steklov. 66 (1962), 364-383 (in Russian).

[5] T. Kobayashi and W. M. Zajączkowski, On global motion of a compressible barotropic viscous fluid with boundary slip condition, Appl. Math. (Warsaw) 26 (1999), 159-194.

[6] L. Landau and E. Lifschitz, Hydrodynamics, Nauka, Moscow, 1986 (in Russian).

[7] J. Serrin, Mathematical Principles of Classical Fluid Mechanics, Handbuch der Physik, Bd. VIII/1, Berlin, Springer, 1959.

[8] V. A. Solonnikov, A priori estimates for second order parabolic equations, Trudy Mat. Inst. Steklov. 70 (1964), 133-212 (in Russian); English transl.: Amer. Math. Soc. Transl. (2) 65, 51-137.

[9] V. A. Solonnikov and A. Tani, Evolution free boundary problem for equations of motion of viscous compressible barotropic liquid, in: Lecture Notes in Math. 1530, Springer, 1992, 30-55. 
[10] V. A. Solonnikov and A. Tani, Free boundary problem for a viscous compressible flow with a surface tension, in: Constantin Carathéodory: An International Tribute, T. M. Rassias (ed.), World Sci., 1991, 1270-1303.

[11] E. Zadrzyńska, On nonstationary motion of a fixed mass of a general viscous compressible heat conducting capillary fluid bounded by a free boundary, Appl. Math. (Warsaw) 25 (1999), 489-511.

[12] - Evolution free boundary problem for equations of viscous heat-conducting capillary fluids, Math. Methods Appl. Sci. 24 (2001), 713-743.

[13] E. Zadrzyńska and W. M. Zajączkowski, Local existence of solutions of a free boundary problem for equations of compressible viscous heat conducting capillary fluids, J. Appl. Anal. 6 (2000), 227-250.

[14] - - - On some inequalities for solutions of equations describing the motion of a viscous compressible heat-conducting capillary fluid bounded by a free surface, Appl. Math. (Warsaw) 28 (2001), 31-53.

[15] W. M. Zajączkowski, On nonstationary motion of a compressible barotropic viscous capillary fluid bounded by a free surface, SIAM J. Math. Anal. 25 (1994), 1-84.

Ewa Zadrzyńska

Faculty of Mathematics and Information Sciences Warsaw University of Technology

pl. Politechniki 1

00-661 Warszawa, Poland

E-mail: emzad@impan.gov.pl
Wojciech M. Zajączkowski Institute of Mathematics Polish Academy of Sciences Śniadeckich 8 00-950 Warszawa, Poland E-mail: wz@impan.gov.pl

Military University of Technology Kaliskiego 2 00-908 Warszawa, Poland

Current address: ICM, Interdisciplinary Centre for Mathematical and Computational Modelling

Warsaw University Pawińskiego 5a 02-106 Warszawa, Poland

Received on 8.11.2001;

revised version on 17.5.2002 